University of Louisville

ThinkIR: The University of Louisville's Institutional Repository

Electronic Theses and Dissertations

$12-2016$

\title{
Using a mouse model to investigate male bias in autism spectrum disorder incidence.
}

Elizabeth Ann Gordon

University of Louisville

Follow this and additional works at: https://ir.library.louisville.edu/etd

Part of the Endocrinology Commons

\section{Recommended Citation}

Gordon, Elizabeth Ann, "Using a mouse model to investigate male bias in autism spectrum disorder incidence." (2016). Electronic Theses and Dissertations. Paper 2608.

https://doi.org/10.18297/etd/2608

This Doctoral Dissertation is brought to you for free and open access by ThinkIR: The University of Louisville's Institutional Repository. It has been accepted for inclusion in Electronic Theses and Dissertations by an authorized administrator of ThinkIR: The University of Louisville's Institutional Repository. This title appears here courtesy of the author, who has retained all other copyrights. For more information, please contact thinkir@louisville.edu. 


\title{
USING A MOUSE MODEL TO INVESTIGATE MALE BIAS IN AUTISM SPECTRUM DISORDER INCIDENCE
}

\author{
By \\ Elizabeth Ann Gordon \\ B.S., Murray State University, 2008 \\ M.P.H., University of Louisville, 2010
}

\begin{abstract}
A Dissertation
Submitted to the Faculty of the

College of Arts and Sciences of the University of Louisville in Partial Fulfillment of the Requirements

for the Degree of
\end{abstract}

Doctor of Philosophy in Biology

Department of Biology

University of Louisville

Louisville, Kentucky

December 2016 
Copyright 2016 by Elizabeth Ann Gordon

All rights reserved 



\title{
USING A MOUSE MODEL TO INVESTIGATE MALE BIAS IN AUTISM SPECTRUM DISORDER INCIDENCE
}

\author{
By \\ Elizabeth Ann Gordon \\ B.S., Murray State University 2008 \\ M.P.H., University of Louisville 2010
}

A Dissertation Approved on

November 11, 2016

by the following Dissertation Committee:

Dissertation Director

Dr. Cynthia Corbitt

Dr. Keith Lyle

Dr. Holly Swain Ewald

Dr. Mark Running

Dr. Manuel Casanova 


\section{ABSTRACT \\ USING A MOUSE MODEL TO INVESTIGATE MALE BIAS IN AUTISM SPECTRUM DISORDER INCIDENCE}

Elizabeth Ann Gordon

November 11, 2016

The review (Chapter 1) overviews our current understanding of the sex bias in autism spectrum disorders (ASD), whereby males are 4 times more likely to develop the condition. The longstanding uncertainty surrounding the etiology of autism can be informed by inclusion of both sexes in future research to better understand the mechanism responsible for this sex bias. Both clinical studies and animal models are useful tools to elucidate the underlying cause(s) of sexual dimorphic morphological, physiological, and behavioral differences in the ASD phenotype.

Sex differences in social behaviors are common across species, underscoring the importance of assessing baseline sex differences in behavior, especially when sexually dimorphic effects on behavior are expected. I examined pre-pubertal behavior (Chapter 2) in 3 strains of mice (C57BL/6, CFW, and CF1) using a wheel-running assay developed to be relevant to autism-like behaviors. The results indicated no sex differences in wheel running behaviors or social interaction with all mouse strains displaying typical wheel running behavior, i.e. more time running on the wheel when it would turn and increased performance of other activities when it was jammed. 
Studies have found that elevated levels of fetal testosterone (fT) are correlated with later ASD diagnosis. Prenatal valproic acid (VPA) treatment is a common animal model of ASD in rodents, mimicking many of the features of ASD including increased male vulnerability. I used the VPA model (Chapter 3) to evaluate sex-specific responses to prenatal VPA treatment, and to assess if masculinization of females with perinatal testosterone propionate (TP) after prenatal VPA treatment would masculinize their susceptibility for abnormalities in development relevant to ASD. My findings confirmed that VPA exposure results in impaired motor development abilities, and provide novel evidence that the combined effects of VPA, TP, and maleness (triple hit) led to more severe motor development deficiencies in some tasks. I also present evidence that the triple hit resulted in reduced anxiety, but elevated cognitive rigidity and self-grooming compared to control females. The results provide some evidence for increased vulnerability of triple hit males to behavioral deficits relevant to ASD, but more research is needed to better understand this effect. 


\section{TABLE OF CONTENTS}

\section{PAGE}

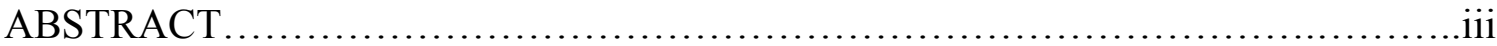

LIST OF FIGURES.............................................................. viii

CHAPTER

PAGE

I. Sex differences in ASD: evidence from clinical studies and animal models

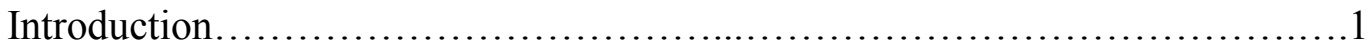

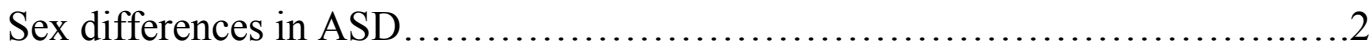

ASD prevalence by sex..............................................

Diagnostic practices that may affect the sex ratio........................ 3

Sex differences in neurobiology and behavior..........................5

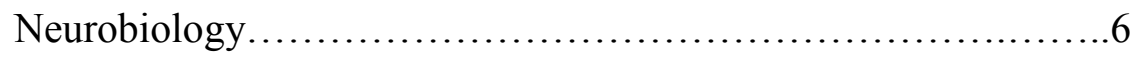

Behavior................................................ 9

Proposed mechanisms for sex differences....................................12

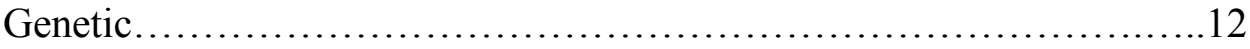

Prenatal steroid hormones...............................................15

Environmental mechanisms............................................19

Animal models - sex differences in phenotypes..............................23

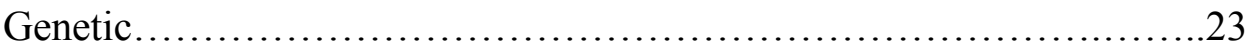

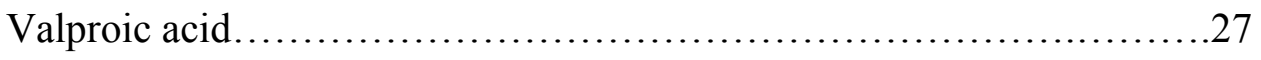

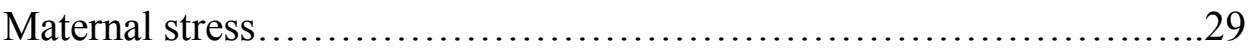


Conclusions

Organization of Dissertation.....

II. Investigation of pre-pubertal sex differences in wheel running and social behavior in three mouse strains

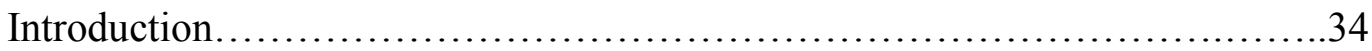

Material and Methods................................................. 37

Animals............................................................

Test Procedure.................................................... 38

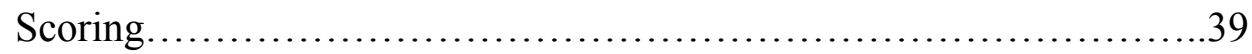

Statistics...................................................40

Results..........................................................4

Latency of Behavior.......................................4

Duration of Behavior.......................................42

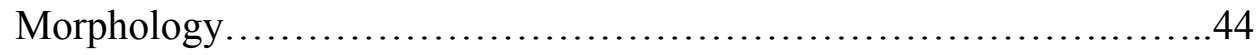

Discussion.............................................................. 44

III. Evaluating sex differences and the effect of perinatal testosterone in a mouse model of autism

Introduction....................................................5 57

Material and Methods................................................60

Animals.....................................................60

Behavioral Testing.........................................62

Brain Measures..............................................66

Statistics................................................66

Results............................................................. 67

Motor Development Tests.....................................67 
Open Field/Elevated Plus Maze.......................................68

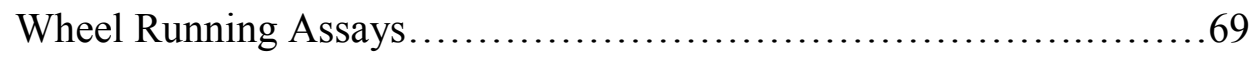

Brain Measures............................................................. 72

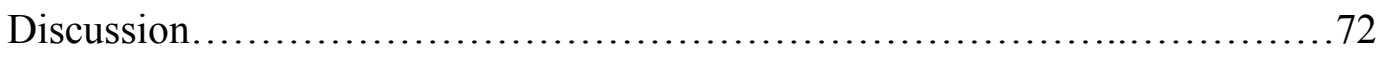

IV. Summary and Future Directions

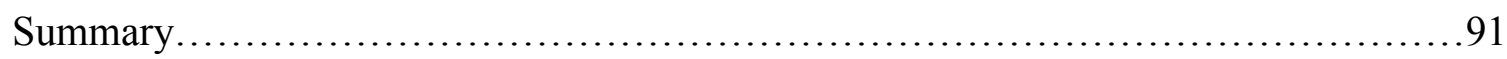

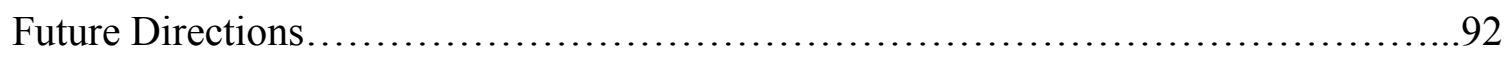

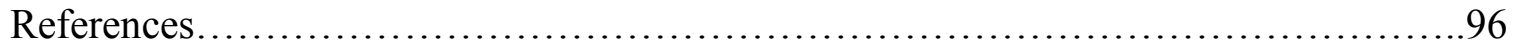

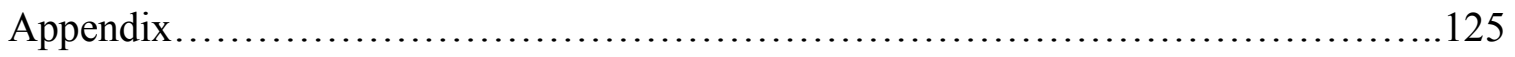

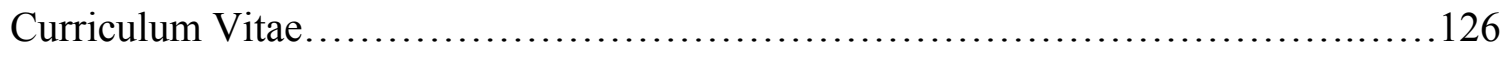




\section{LIST OF FIGURES}

FIGURE PAGE

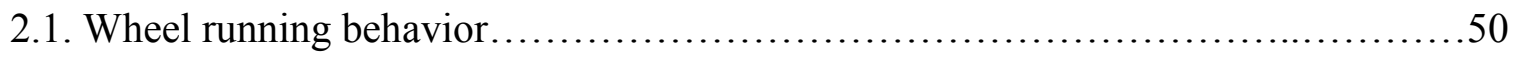

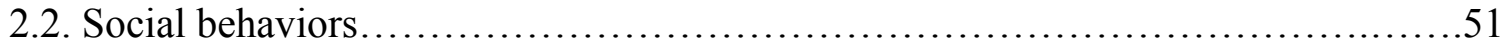

2.3. Duration of sterotypical behaviors ........................................5

2.4. Duration of all behaviors by sex and day ......................................5

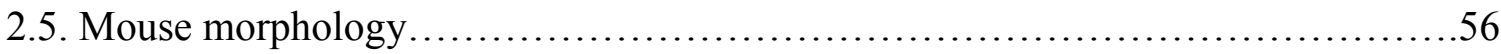

3.1. Duration or score of motor development behaviors by day.......................86

3.2. Frequency, duration, and velocity measures from EPM........................87

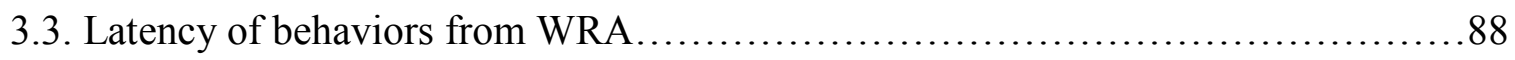

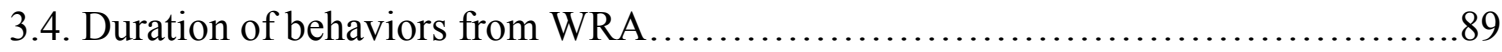




\section{CHAPTER 1}

\section{SEX DIFFERENCES IN ASD: EVIDENCE FROM CLINICAL STUDIES AND}

\section{ANIMAL MODELS}

\section{Introduction}

Autism spectrum disorders (ASD, also referred to as autism) are behaviorally defined by the Diagnostic and Statistical Manual of Mental Disorders 5th edition (DSMV) as characterized by deficits in social communication and social interaction, including social-emotional reciprocity, nonverbal communication, and development and maintenance of relationships (Diagnostic and Statistical Manual of Mental Disorders, 2013). Diagnosis also requires restricted, repetitive patterns of behavior, interests, or activities, which may include hyper- or hypo-reactivity to sensory input (Diagnostic and Statistical Manual of Mental Disorders, 2013). The autism phenotype is highly heterogeneous with a wide range of symptoms, severities, and co-morbidities, including intellectual impairment, anxiety, and epilepsy (Amaral, Schumann, \& Nordahl, 2008). Although agreement has yet to be reached regarding the level of genetic contribution to autism (estimates range from $40 \%$ to $90 \%$ (Rybakowski et al., 2016)), the current consensus is that in most cases the interaction of genes and the environment result in the ASD phenotype (Tordjman et al., 2014). Due to the nature of the early onset of autism within the first 3 years of life, most environmental etiologies are implicated prenatally or during early development (Casanova, 2007; Tordjman et al., 2014). Autism is heavily 
biased towards males (Auyeung, Taylor, Hackett, \& Baron-Cohen, 2010), with ratios ranging from 4:1 for classic autism to 11:1 in individuals with Asperger Syndrome (Baron-Cohen et al., 2011). This sex bias could hold important information about the mechanisms underlying ASD, but the cause(s) of this bias remains unknown. Just as ASD is a multifactorial condition resulting from the interaction of multiple factors, it is likely that the sex bias presented in ASD is also complex and is multiply determined.

\section{Sex differences in ASD}

\subsection{ASD prevalence by sex}

The prevalence of autism has climbed over past decades, with the latest estimates from 2012 data at 1 in 68 eight year olds affected, which is the same as in 2010, but roughly $30 \%$ higher than that of 2008 ( 1 in 88 ) and 120\% higher than 2002 (1 in 150), according to the CDC's Autism and Developmental Disabilities Monitoring (ADDM) Network (Center for Disease Control and Prevention, 2016). Males continue to significantly outnumber females in diagnoses. Sex bias was noted in the initial descriptions of autism with eight of eleven cases described by Kanner (1943) and all four cases reviewed by Asperger (1944) being male. This sex difference is a distinction that holds true today, although recent work has begun to question the implications and true extent of this difference.

Assessments of the sex ratio in ASD vary widely, from conservative estimates of the M:F ratio around 2:1 (Eric Fombonne, 2005; Idring, 2014) to estimates as high as 16:1 (E. Fombonne, 2009; Kreiser \& White, 2014). The heterogeneous nature of ASD leads to variability of the sex ratio with males over-represented among high functioning cases, but the sexes being more equally represented among those with severe intellectual 
disability (ID) (M. C. Lai, Lombardo, Auyeung, Chakrabarti, \& Baron-Cohen, 2015; Werling \& Geschwind, 2013). However, the most commonly cited sex ratio for classic autism is 4:1 (E. Fombonne, 2003; Eric Fombonne, 2005; Kreiser \& White, 2014; Werling \& Geschwind, 2013), with higher estimates around 11:1 to 16:1 in individuals with high functioning ASD, such as Asperger Syndrome (Baron-Cohen et al., 2011; Kreiser \& White, 2014; M. C. Lai, Lombardo, et al., 2015).

\subsection{Diagnostic practices that may affect the sex ratio}

Ascertainment procedures likely contribute to the variability in the sex ratio (Alycia K. Halladay, 2015; Hiller, Young, \& Weber, 2014; M. C. Lai, Lombardo, et al., 2015); 2012 data from the CDC's ADDM Network reported prevalence rates by sex were highly variable across sites using different ascertainment procedures (Center for Disease Control and Prevention, 2016). Typically, school or clinic based samples have a higher $\mathrm{M}: \mathrm{F}$ ratio than population screening (Werling \& Geschwind, 2013). Studies identifying cases by screening medical records and government registries have found M:F ratios of 4:1, 3.8:1, and 6:1 (Kohane et al., 2012; D. C. Lai, Tseng, Hou, \& Guo, 2012; YearginAllsopp et al., 2003). Alternately, an epidemiological study of 5,484 children in Finland found a much lower sex ratio, 2:1 (Mattila et al., 2011). Furthermore, a study from South Korea that surveyed from regular schools (general population), as well as special education schools and a disability registry (high risk population) found the M:F ratios were 2.5:1 and 5.1:1 in the general population sample and high-probability group, respectively (Y. S. Kim et al., 2011).

Kreiser and White (2014) summarized the potential for under-identification of females with ASD due to current diagnostic criteria (Kreiser \& White, 2014). Numerous 
studies have reported sex differences in expression and severity of ASD symptoms, including sex differences in social, attentional, and thought problems (Holtmann, Bolte, \& Poustka, 2007); variation in sensory symptoms, socio-communication difficulties, and self-reported autistic traits (M. C. Lai et al., 2011); differences in repetitive stereotyped behavior (RSB) and social problems (Mandy et al., 2012); and severity in cognitive impairment, stereotyped mannerism, preoccupation with parts of objects, and routines/rituals (Nicholas et al., 2008) (see section 2.3 for more detail). Given these sex differences, females are less likely than males to meet diagnostic criteria for autism even when displaying equivalently high levels of ASD traits (Dworzynski, Ronald, Bolton, \& Happe, 2012; Russell, Steer, \& Golding, 2011). Dworzynski et al. (2012) also found the diagnosis rate for girls with high scores on the Development and Wellbeing Assessment (DAWBA) was lower (38\%) than equally high scoring boys $(56 \%)$ on the same assessment tool. Kreiser and White (2014) pointed out that this could indicate that the diagnostic instruments are not as valid for females (Lindsay \& Widiger, 1995). Additionally, there could be under-recognition by clinicians or other referrals sources because of less severe difficulties or failure to display stereotypical autism-like behavior in females (Mandy et al., 2012).

Because of the sex ratio in ASD prevalence, naturally, many studies include more male participants, but others choose to limit their studies entirely to males. A metaanalysis of autism functional magnetic resonance imaging (fMRI) studies found that many failed to report the sex ratio of participants; in research that did, $60 \%$ of studies used only males and the remaining had a sex ratio of 15:1 (Philip et al., 2012). In another meta-analysis focused on brain volumetric measurements of 496 autistics, $89 \%$ of 
participants were male (Via, Radua, Cardoner, Happe, \& Mataix-Cols, 2011). These examples are representative of most studies on ASD. While it may be easier to identify and recruit males for ASD research, this could result in generalizations about our understanding of autism that may be more representative of the male phenotype.

The male bias in ASD prevalence is highly variable across studies and could be exaggerated due to ascertainment bias, under-recognition of females based on diagnostic criteria, and over-representation of males for inclusion in research. The most recent population-based epidemiological studies estimate the M:F ratio is less than the typically reported 4:1 ratio, and is actually around 2:1 to 3:1 (M. C. Lai, Baron-Cohen, \& Buxbaum, 2015). Irrespective of the magnitude of a reporting and diagnostic bias, the evidence clearly points to the existence of a sex bias in autism prevalence. The mechanisms underlying this sex difference may provide information about the etiology and development of ASD.

\subsection{Sex differences in neurobiology and behavior}

Studies evaluating sex differences in neurobiology and brain morphology are limited; however, recent work has highlighted an effect of sex on neural development in ASD (Alycia K. Halladay, 2015; M. C. Lai, Baron-Cohen, et al., 2015; M. C. Lai, Lombardo, et al., 2015; M. C. Lai et al., 2013). Aside from the well documented sex difference in the prevalence of ASD, the only other consistent finding regarding sex is that females with ASD generally have lower intelligence quotient (IQ) scores than males with ASD (Lord \& Schopler, 1985; Lord, Schopler, \& Revicki, 1982; Mandy et al., 2012; Nicholas et al., 2008; Volkmar, Szatmari, \& Sparrow, 1993). Since ASD is diagnosed based on behavioral characteristics, sex differences in behavioral manifestations from this 
condition have been explored, but with inconsistent findings. Regardless, the current evidence on sex differences in brain and behavioral phenotypes are described below.

\subsubsection{Neurobiology}

Ben-Itzchak et al (2013) found that among 663 autistic males and females (age 18 months-15 years), head size, as measured by mean head circumference, was larger in males than females, while microcephaly was more prevalent in females than males (BenItzchak, Ben-Shachar, \& Zachor, 2013). They also noted more frequent minor neurological and musculoskeletal deficits in females, but no difference in severity, cognitive ability, or adaptive functioning were observed between the sexes (Ben-Itzchak et al., 2013). Bloss and Courchesne (2007) evaluated gray and white matter volumes for the entire cerebrum, cerebral lobes, and cerebellum, along with total brain volume in a sample of children with ASD ( $n=36,9$ girls) and neurotypical controls ( $n=27,14$ girls) (Bloss \& Courchesne, 2007). Unlike the differences found in head circumference, the ASD girls in Bloss and Courchesne's study displayed similar size-related abnormalities as ASD boys (Bloss \& Courchesne, 2007). However, they did find further irregularities in ASD girls, such as enlarged white and gray matter volumes in the temporal lobes and reduced cerebellar gray matter volume (Bloss \& Courchesne, 2007).

A study by Schumann et al. (2009) focused on amygdala volumes taken from MRI scans found that among 89 children (mean age=3 years), those receiving an ASD diagnosis ( $n=41,32$ boys) had a larger amygdala when compared to normal developing controls ( $n=39,28$ boys) and that the severity of communication and social deficiencies was related to amygdala size (Schumann, Barnes, Lord, \& Courchesne, 2009). Interestingly, the ASD girls' amygdala volume differed more significantly when 
compared to controls, while the relationship between amygdala size and behavioral impairment was more significant in boys (Schumann et al., 2009). Schumann and colleagues (2010) also performed a longitudinal study evaluating neural growth in children aged 1.5 to 5 years ( $n=85,41$ with ASD and 44 controls) using structural MRI from several time points (Schumann et al., 2010). They found enlarged cerebral gray and white matter in children with ASD by age 2.5 and that all regions measured, except for occipital gray matter, displayed an abnormal growth trajectory in ASD participants over the longitudinal study (Schumann et al., 2010). This study also noted that abnormal growth was pronounced in more brain regions in ASD females compared to ASD males (Schumann et al., 2010).

Recent work by Nordahl et al. (2015) found sex differences in corpus callosum alterations of preschool-aged children with $\operatorname{ASD}(n=139,112$ males) compared with normally developing controls ( $n=82,53$ males) using a longitudinal study design gathering structural and diffusion-weighted images (Nordahl et al., 2015). Children with ASD of both sexes had a reduction in regional volume projecting to the superior frontal cortex; however, there were sexually dimorphic patterns of projection from the corpus callosum subregions to other areas in the frontal cortex with males having reduced callosal region corresponding to the orbitofrontal cortex and females having a reduced callosal region corresponding to the anterior frontal cortex (Nordahl et al., 2015). In addition, they found increased diffusion properties of callosal fibers (mean diffusivity, axial diffusivity, and radial diffusivity) in females, but not males, with ASD relative to controls (Nordahl et al., 2015). 
Lai et al. (2013) evaluated high-functioning adults of both sexes with and without $\operatorname{ASD}(n=120, n=30 /$ group) and compared neuroanatomical features using voxel-based morphometry (VBM) (M. C. Lai et al., 2013). They found nominal spatial overlap in grey and white matter (2.3\% and $1.0 \%$, respectively) between males and females with ASD (M. C. Lai et al., 2013). They suggest this indicates neuroanatomy developed differently in autistic males and females and is altered from the neuroanatomy of same-sex controls (M. C. Lai et al., 2013). The study also reported that atypical grey and white matter areas in females with ASD overlapped with sexually dimorphic areas in controls providing evidence of masculinization of these females (M. C. Lai et al., 2013). A study by Beacher et al. (2012) also found sex-related anatomic features in adults with ASD. They assessed volume measures using structural MRI, regional effects with VBM, and white matter tract integrity using diffusion tensor imaging (Beacher et al., 2012). This group reported associations between diagnosis and sex on measures of total white matter volume, regional gray matter volume in the right parietal operculum, and white matter water diffusion anisotropy in the body of the corpus callosum, cingulum, and corona radiata (Beacher et al., 2012). Specifically, sex-related differences in healthy controls $(n=30)$ including larger fractional anisotropy and total white matter volume in males were reduced or absent in ASD participants ( $n=28)$ (Beacher et al., 2012).

Overall, the current literature strongly supports sex differences in neural development of children across many regions of the brain, including the temporal lobes (Bloss \& Courchesne, 2007), cerebellum (Bloss \& Courchesne, 2007), amygdala (Schumann et al., 2009), and corpus callosum (Nordahl et al., 2015). In addition, there is evidence from studies in adults that the neuroanatomy of grey and white matter is altered 
in males and females with ASD (Beacher et al., 2012; M. C. Lai et al., 2013), and that ASD females' brains show masculinization (M. C. Lai et al., 2013). Taken together, this evidence underscores the importance of evaluating sex differences in neurobiology and that there could be both common and unique etiologies between the sexes in the development of ASD.

\subsubsection{Behavior}

A study by Lord et al. (1982) of almost 500 children (age 3-8, 80\% male) with mild to severe autism suggested that males performed better on eye-hand integration and perception skills using the Psychoeducational Profile (PEP) and had higher nonverbal IQ social quotient and Peabody Picture Vocabulary Test (PPVT) scores than females. Controlling for nonverbal IQ resulted in loss of significance on the PPVT and on eyehand integration and perception scales, but the main effect of sex remained. Males also displayed increased unusual visual responses and more stereotypic play compared to females (Lord et al., 1982).

Using several standard measures of developmental and autistic behavior, Hartley and Sikora (2009) found patterns of development, autism symptoms, and behavior problems were similar in both sexes of young children aged 1.5-3.9 ( $n=199,157$ boys) with ASD. However, girls with ASD showed more marked communication deficits, additional sleep issues, and increased anxious and depressed affect compared to boys, while boys displayed greater restricted, repetitive behavior (RRB) compared to girls (Hartley \& Sikora, 2009). Another study of toddlers ( $n=90,68$ boys, mean age 28 months) also found that girls showed weaker verbal skills, yet displayed stronger visual reception skills compared to boys (Carter et al., 2007). In addition, they reported that 
boys had superior motor skills and more advanced social competence ratings than girls; however, they failed to find a sex difference in RRB (Carter et al., 2007).

A large study of children and adolescents with a diagnosis of high functioning ASD ( $\mathrm{n}=325,52$ females) by Mandy et al. (2012) reported males to have more RRB by parent report and direct observation using autism diagnostic observation schedule scores. Analysis of associated features found that there were no sex differences for visuo-spatial impairment, gross motor impairment, auditory sensitivity, feeding difficulties or sensitivity to sound. Females were reported to have better fine motor skills than males, but there was no difference in this measure in the younger half of the sample. Parents reported higher levels of emotional symptoms for girls than boys on the Strengths and Difficulties Questionnaire (SDQ), but all other problems were the same. According to teacher report on the same measure, males were had a higher total problem score with greater difficulties with hyperactivity/inattention and prosocial behavior (Mandy et al., 2012).

A study of 83 adults (45 male) aged 18-45 with high-functioning ASD or Asperger's syndrome found that during interpersonal interaction, females showed less autistic behavior than males in socio-communication and RRB domains, but had more lifetime sensory symptoms and self-reported autistic traits. However, there was no sex difference in self-reported systemizing, empathy, anxiety, depression, or obsessivecompulsive symptoms (M. C. Lai et al., 2011). McLennan et al. (1993) conducted a study with 21 autistic females between the ages of 6 and 36, using 21 male autistic subjects matched for age, diagnosis, and IQ. Overall, they found a significant main effect of sex in social interaction measures with males displaying more abnormal behavioral 
development than females. However, more problems were identified in females when assessing current friendships. Unlike some other studies, no sex difference for RRB or nonsocial aspects of communication were found. Looking at the data within a more narrow age range, males were also discovered to have more severe communication and social problems at age 3 and ages 4-5 (McLennan, Lord, \& Schopler, 1993).

Other studies have failed to find sex differences in the key features of ASD. Holtmann et al. (2007) conducted a study of 23 males and 23 females (mean age $\sim 12$ years) with high-functioning ASD and reported no differences between the sexes in any of the autism core symptoms (social interaction, communication, RRB). According to parent report, though, females displayed more symptoms than males in social, attention, and thought problem domains, such as peer relation impairments, social immaturity, social dependence, inattentive, impulsive, or hyperactive behavior; however, parents' perceptions could be influenced by sex-biased expectations of behavior (Holtmann et al., 2007). Another study comparing 18 males and 18 females with an ASD diagnosis also found no significant differences in autism symptomatology using 2 autism diagnostic rating scales (Pilowsky, Yirmiya, Shulman, \& Dover, 1998). In a larger study comparing 156 males and 43 females with autism, Volkmar and colleagues (1993) reported no sex differences in the severity of autism symptoms, aside from the fact that autistic females were more likely to have a lower IQ (Volkmar et al., 1993).

Many researchers have explored sex differences in behavioral manifestations of autism, but with inconsistent and sometimes conflicting results. The variable results among studies could be the consequence of numerous factors including small sample size, difficulty recruiting adequate numbers of females, failure to match for factors like 
age, IQ, and changes in diagnostic criteria over time. Aside from issues with study design, the heterogeneous nature of ASD as a "spectrum" disorder can make reaching a consensus difficult, especially when considering sex differences in behavior. However, regardless of these variations, the body of literature continues to indicate a higher prevalence in the disorder for males, with differences in reporting or diagnosis perhaps accounting for the discrepancy in the ratio of 2:1 to 4:1. It is also clear that in general, females with ASD have lower IQ scores than males with ASD, and that many studies have found variability in behavioral manifestations of the disorder. As more studies work to include females and control for bias, a clearer picture of the true sex difference should emerge.

\section{Proposed mechanisms for sex differences}

\subsection{Genetic}

The inheritance patterns of ASD from family and twin studies, and their implication that genetic variation confers over half the risk for developing ASD (De Rubeis \& Buxbaum, 2015), prompted extensive research into identifying the genes that may be significant causative factors (Muhle, Trentacoste, \& Rapin, 2004). Genetic studies have identified over 200 autism susceptibility genes(Tordjman et al., 2014), with estimates that there could be up to 1200 ASD risk genes (De Rubeis \& Buxbaum, 2015) associated with this condition, and confirmed that ASD is a complex, multifactorial disorder resulting from simultaneous action of genetic variants on multiple genes (polygenetic) and by environmental factors (Tordjman et al., 2014). Given the involvement of genetic mechanisms, an obvious explanation for the sex bias would be a sex chromosome-linked effect. 
Due to the nature of mammalian inheritance, such that males have only one $\mathrm{X}$ chromosome, many geneticists have explored genes on the $\mathrm{X}$ chromosome. Genetic studies in those with ASD have found cases of associated loci on the X chromosome including 4 genes: Fragile X mental retardation (Fmrl), Mecp2, and neuroligin (Nlgn) 3 and 4 (Paul H. Patterson, 2006). Fragile X syndrome (FXS) is caused by mutations in Fmrl and includes some symptoms similar to those in autism (mental retardation, attention deficit, anxiety, seizures, stereotypic behaviors, and communication deficits). Estimates indicate that $10-40 \%$ of those with FXS also meet criteria for autism diagnosis; however, only $2-8 \%$ of autism cases involve FXS (Tordjman et al., 2014; Wassink, Piven, \& Patil, 2001). Mutations in neuroligin 3 and 4 are associated with autism, as well as mental retardation (Jamain et al., 2003; Laumonnier et al., 2004). NLGN 3 and 4 promote protein processing and stimulation of synapse formation, aiding in the development of connectivity within the brain (Chih, Afridi, Clark, \& Scheiffele, 2004; Comoletti et al., 2004). Mutations in Mecp2, the gene responsible for Rett syndrome, are also found in those with autism and the two conditions have similar symptoms (Glaze, 2004). MECP2 regulates gene expression (e.g., Bdnf) and may link synaptic activity and transcription (Neul \& Zoghbi, 2004). An X chromosome-linked effect is the most obvious explanation for the sex bias; however, all cases of associated loci on the $\mathrm{X}$ chromosome (Fmr1, Mecp2, Nlgn 3 and Nlgn 4) cause significant intellectual disability that is not seen across the spectrum of autism cases, and the number of ASD cases linked with these genes represents only a small fraction of total ASD cases, not large enough to explain the sex bias (Paul H. Patterson, 2006; Werling \& Geschwind, 2013). 
Additionally, ASD transmission through families does not generally follow an X-linked pattern of inheritance (Todd, 1993; Werling \& Geschwind, 2013).

Inherited mutations on the $\mathrm{Y}$ chromosome are also of interest given mammalian inheritance, yet this explanation is also implausible because father-to-son transmission of a severe autism phenotype is rarely documented (Beaudet, 2006), and it would not explain ASD in girls. However, those severely affected by the disease presumably have a reduction in reproductive fitness, and the possibility of transmission in those with milder phenotypes is unknown (Beaudet, 2006). Longstanding mutations on the Y chromosome with association to autism are also improbable based on evidence comparing $\mathrm{Y}$ chromosome haplotypes in autism to controls (Jamain et al., 2002).

Another mechanism used to elucidate effect of sex chromosomes on ASD risk is to study individuals with sex chromosome aneuploidies. These individuals often have increased autism-like symptoms and are more likely to develop autism (Schaafsma \& Pfaff, 2014). In Klinefelter syndrome (47, XXY) males often have language-based learning disabilities (Geschwind, Boone, Miller, \& Swerdloff, 2000) and display social withdraw and higher levels of autistic traits (Tartaglia, Cordeiro, Howell, Wilson, \& Janusz, 2010; van Rijn \& Swaab, 2011). According to Bishop et al. (2011) 47, XXY and 47, XYY boys are diagnosed with autism at a higher rate than the general population as a whole, $10 \%$ and $20 \%$, respectively. Both XXY males and XYY males, along with XXX females, are registered for special education needs (SEN) and speech and language therapy at a much higher rate than the general population, but XXX females do not display an increased rate of ASD (Bishop et al., 2011). Females with Turner syndrome $(45, \mathrm{XO})$ are more likely than XX girls to be diagnosed with autism (3\%) (Creswell, 
1999; Werling \& Geschwind, 2013) and have problems with social relationships and peer interactions (McCauley, Ito, \& Kay, 1986). Based on observations that XXX females have no increased autism risk while XXY males have a slight increased risk (10\%) and XYY males have a significant increased risk (20\%), along with increased risk in XY males compared to XX females, Werling and Geschwind (2013) proposed that the Y chromosome could be a risk factor for ASD. Considering that XO females also have an increased risk of autism, albeit smaller (3\%), Werling and Geschwind (2013) go on to assert that a second X chromosome could be protective (Werling \& Geschwind, 2013). Furthermore, it was observed in females with Turner syndrome that those with a maternally inherited X chromosome had reduced social cognition when compared with XO females with a paternally inherited X chromosome (Donnelly et al., 2000; Skuse et al., 1997). Given this, others have proposed that it is imprinted genes that are expressed from the paternal, but not maternal, $\mathrm{X}$ chromosome that are protective against ASD (Skuse, 2000). This is supported by increased rates of autism in Klinefelter syndrome cases, given that $40-50 \%$ result from maternal nondisjunction, meaning that approximately half of these cases lack the potentially protective paternal X. Since all XYY cases lack paternal X expression, along with 2 Y chromosomes, the observed higher rate in XYY cases supports the protective paternal X hypothesis (Werling \& Geschwind, 2013).

\subsection{Prenatal steroid hormones}

Although the presence of the $\mathrm{Y}$ chromosome itself might not explain ASD patterns, the sex-determining region of the Y chromosome (Sry) gene sets hormonallybased differentiation in motion by leading to testis formation. Sexual differentiation of 
the brain begins during prenatal development following the production of steroid hormones, such as testosterone (T), by the fetal testes (Lenz, Nugent, \& McCarthy, 2012). The classic Organizational-Activational Hypothesis proposed by Phoenix et al. (1959), states that gonadal hormones organize brain regions during a critical period in development, which produces differences in brain development and behavior between the sexes (Phoenix, 1959). More recently, sex differences in brain molecular, cellular, and neural systems have been described (Spring, Lerch, \& Henkelman, 2007), including roles for de novo neurosteroidogenesis (Lenz et al., 2012). These sexual dimorphisms can affect how each sex develops a disease, their signs and symptoms of that disease, and their response to therapy (Becker et al., 2005). The effect of endogenous steroids on sexual differentiation of the brain, early life diagnosis of ASD, and higher incidence of ASD in males, has launched investigations aimed at understanding the potential role of steroid hormones in differential susceptibility of the sexes to ASD (Alycia K. Halladay, 2015; M. C. Lai et al., 2013; Schaafsma \& Pfaff, 2014; Werling \& Geschwind, 2013). Multiple studies have found evidence that steroid hormones are atypical in those with ASD (Schaafsma \& Pfaff, 2014). Pre-pubertal autistic children (both boys and girls) displayed increased levels of serum dehydroepiandrosterone (DHEA), serum testosterone, and androstenedione, while serum follicle-stimulating hormone (FSH) levels were decreased, compared to age and sex-specific normal lab reference ranges (Geier \& Geier, 2006, 2007). In a study comparing individuals with Asperger's syndrome or highfunctioning autism to age and IQ-matched controls, serum androstenedione levels were elevated in the ASD group (Ruta, Ingudomnukul, Taylor, Chakrabarti, \& Baron-Cohen, 2011). Salivary testosterone levels were also elevated in pre-pubertal children with 
autism and Asperger syndrome and pubertal patients with autism compared to controls (Schmidtova, 2010). In addition, in a survey of women, those with ASD reported androgen-related conditions, including hirsutism, irregular menstrual cycles, dysmenorrhea, polycystic ovarian syndrome (PCOS), severe acne, tomboyism, bisexuality/asexuality, and a family history of ovarian, uterine, or prostate cancer, tumors, or growths significantly more often than controls (Ingudomnukul, Baron-Cohen, Wheelwright, \& Knickmeyer, 2007).

Some researchers propose that the male bias in ASD diagnosis manifests from an extreme expression of the psychological and physiological properties of the typical male brain. This hypothesis suggests males require slight psychological and physiological changes from normal males, while differences in females from their non-ASD counterparts must be more extreme, to display the ASD phenotype; this is known as the Extreme Male Brain (EMB) Theory (Baron-Cohen et al., 2011). Baron-Cohen and colleagues propose that the EMB phenotype could result from elevated levels of fetal testosterone (fT), an androgen receptor abnormality, or sexually dimorphic gene expression unrelated to fT (Baron-Cohen, Knickmeyer, \& Belmonte, 2005), but their recent evidence supports the elevated fT premise (Baron-Cohen et al., 2014).

Multiple conditions result in an altered hormonal environment during fetal development, providing insight into the effect that prenatal hormones have on the developing brain (Gore, Martien, Gagnidze, \& Pfaff, 2014). In females with congenital adrenal hyperplasia $(\mathrm{CAH})$, prenatal adrenal androgen production is elevated, which has been associated with masculinized behavior and cognitive skills, including male preferred playmates (Hines \& Kaufman, 1994) and masculine attributes on some visual-spatial 
tasks (Hines et al., 2003) (but see (McGuire, Ryan, \& Omenn, 1975)). There is further evidence in females with $\mathrm{CAH}$ of increased autistic-like behavioral and cognitive characteristics; for example, $\mathrm{CAH}$ females exhibited higher scores on the autismspectrum quotient (AQ) than their unaffected female siblings and had reduced vocabulary scores (Knickmeyer et al., 2006). In children whose mothers had polycystic ovary syndrome with elevated blood testosterone during pregnancy, total AQ scores were higher compared to controls, with many areas only significantly affected in daughters (Palomba et al., 2012).

Furthermore, numerous studies have analyzed fT levels from amniotic fluid samples in both sexes and found a relationship between fT levels and ASD indicators, including autistic traits in toddlers (Auyeung et al., 2010), quality of social relationships and restricted interests in boys at age 4 (Knickmeyer, Baron-Cohen, Raggatt, \& Taylor, 2005), and scores on two measures of empathy in children ages 6-8 (Chapman et al., 2006). A recent study found that fetal steroidogenic activity (including $T$ and hormones along the $\Delta 4$ biosynthetic pathway to $\mathrm{T}$ ) is elevated (above that of controls) in utero for boys and girls later diagnosed on the autism spectrum (Baron-Cohen et al., 2014). Given that amniocentesis is primarily performed in high risk pregnancies, a longitudinal study using umbilical blood taken at birth in both low- and high-risk pregnancies was performed with results indicating no link between autistic behaviors or autism diagnosis and perinatal testosterone levels (Whitehouse et al., 2012). However, T levels generally peak during mid-pregnancy and are relatively low around the time of birth in humans (Bao \& Swaab, 2011; Swaab, 2004), so the window of vulnerability for developing autism may have been missed in this study. 


\subsection{Environmental mechanisms}

Many modern diseases that significantly affect public health are a result of the interaction of multiple genes with the environment. For example, heart disease, diabetes, cancer, spina bifida/anencephaly, pyloric stenosis, cleft palate, COPD, mood disorders, autism and others are thought to be complex conditions (Saito, 2011; Stolk et al., 2008). Results from the largest population-based twin autism study to-date suggests that environmental factors common to twins, not genetic factors, are responsible for explaining the majority (about 55\%) of the liability to autism (Hallmayer et al., 2011). Many environmental mechanisms have been implicated in the development of ASD, and some present potential mechanisms for the sex bias.

Human studies, as well as rodent models, demonstrate that prenatal stress impacts the hypothalamic-pituitary-adrenal (HPA) axis, which can increase glucocorticoid secretion even in adulthood and cause structural changes in the hippocampus, frontal cortex, amygdala, and nucleus accumbens (Weinstock, 2008). Stress during pregnancy is also shown to produce poorer cognitive outcomes in infants as measured by the Bayley Mental Developmental Index scores (Markham, Taylor, Taylor, Bell, \& Koenig, 2010) and is linked to increased susceptibility to cognitive and emotional disorders, such as depression, anxiety, schizophrenia, and autism (Mueller \& Bale, 2007, 2008). Many of the diseases mentioned above that are associated with prenatal or maternal stress show a sex bias in incidence (Mueller \& Bale, 2008).

Although the precise mechanism of immune dysfunction in those with ASD remains unclear, research points to a link between the immune system and the pathogenesis of autism (Cohly \& Panja, 2005). A review by Rossignol and Frye (2012) of 
physiological abnormalities in autism found that $95 \%$ of publications implicated an association between ASD and immune dysregulation or inflammation (416 out of 437 publications) (Rossignol \& Frye, 2012). Further evidence suggests that microglial and astrocyte activation, along with cytokine up-regulation, are present in the brain and cerebrospinal fluid of autistic patients (P. H. Patterson, 2009). Maternal immune activation (MIA) has been explored as a leading environmental factor that could increase the risk of ASD by activating microglia and stimulating secretion of pro-inflammatory cytokines (P. H. Patterson, 2009). In a nation-wide study of children born in Denmark between 1980 and 2005, admission to the hospital due to maternal viral infection in the first trimester and maternal bacterial infection in the second trimester were found to be associated with diagnosis of ASD in the offspring (Atladottir et al., 2010). In addition, maternal rubella infection is also associated with neurological and behavioral problems including autism (Chess, Fernandez, \& Korn, 1978); data from a rubella epidemic found the incidence of autism diagnosis increased over 200-fold above normal for offspring exposed prenatally (Chess, 1977).

Associations of maternal infections, inflammation, and ASD diagnosis lead to questions regarding possible mechanisms leading to the sex bias in ASD. Interestingly, immune cells in the brain are implicated in the development of sexually dimorphic brain regions. To date, there are no clinical studies in this area given that most of the research into the impact of sex steroid hormones on brain development is carried out in animals (primarily rodents) (Lenz et al., 2012; McCarthy, Arnold, Ball, Blaustein, \& De Vries, 2012; Park, Baum, Paredes, \& Tobet, 1996; Tobet, Basham, \& Baum, 1993). However, connections between immune activation and mechanisms leading to increased 
vulnerability to ASD in males can be informed by research using rodent models. For example, the pre-optic area (POA) is known to be a highly sexually dimorphic region with male rats displaying more densely populated dendritic spines (Amateau \& McCarthy, 2002). Lenz et al. (2013) recently demonstrated that male rats had double the number of ameboid microglia in their POA, and these microglia also displayed a more activated morphological appearance compared to females (Lenz, Nugent, Haliyur, \& McCarthy, 2013). In addition, estradiol treatment of females masculinized microglial number and morphology, resulting in masculinization of dendritic spine density and male-typical adult copulatory behavior (note: in rodents testosterone from the male gonad is converted to estradiol, a masculinizing agent, in the brain) (Lenz et al., 2013). Thus, testosterone (via estradiol in rodents) increases the number and active morphologic profile of microglia in the male neonatal brain. Human studies in those with sex chromosome aneuploidies or conditions that alter hormonal milieu (i.e., $\mathrm{CAH}$ ) have identified similar mechanisms by which sex steroid hormones affect brain development with testosterone as the main masculinizing agent (Bao \& Swaab, 2011; Swaab, 2004). This evidence offers a link between fetal immune response pathways and masculinization, providing mechanisms whereby maternal immune activation of fetal microglia could have sex-specific effects on brain development, including susceptibility to neurodevelopmental disorders like ASD (Schaafsma \& Pfaff, 2014).

Another environmental factor that has been associated with ASD development is advanced parental age ( $\geq 35$ for mothers and $\geq 40$ for fathers) (Durkin et al., 2008). Furthermore, some studies indicate that the sex ratio of children with an ASD diagnosis can be influenced by parental age (Schaafsma \& Pfaff, 2014). In a historical birth cohort 
study, risk of ASD increased significantly with each 10-year increase in both maternal and paternal age; associations with parental age was slightly stronger for girls, but the sex difference failed to reach statistical significance (Croen, Najjar, Fireman, \& Grether, 2007). Another historical population-based cohort found a significant association between advancing paternal, but not maternal, age and risk of ASD, where children of men $\geq 40$ were 5.75 times more likely to have ASD than children of men $<30$. Interestingly, the M:F ratio in children with ASD whose fathers were 40 years or older (2.3:1) was lower than sex ratio of fathers younger than 40 (5.6:1); however, the sample was too small to test for statistical significance (Reichenberg et al., 2006). Finally, in a study of families with ASD cases, the M:F ratio decreased significantly with increasing paternal age even after adjusting for maternal age (6.2:1 from fathers $<30$ to 1.2:1 from fathers aged $>45$ ); however, no significant relationship was found between maternal age and M:F ratio (Anello et al., 2009). This implies that younger parental age is associated with the typical male predominated sex bias in ASD; however, as parental age increases, especially of fathers, this results in a more even sex distribution, or more cases of ASD in females.

Numerous studies have examined potential genetic, hormonal, and environmental mechanisms for the development of sex differences in ASD. While some X chromosomelinked cases have been explored, their infrequency limits their influence on the overall sex difference in prevalence. However, more can be learned from sex chromosome aneuploidies on the impact of sex chromosomes on this bias, specifically, if the $\mathrm{Y}$ chromosome is a risk factor for ASD (i.e., beyond the SRY-directed formation of the testis) and if the paternal X chromosome offers protective effects. A significant body of 
evidence supports elevated levels of both pre- and post-natal testosterone as increasing one's risk for ASD, but the mechanism for this occurrence and its level of influence on the overall sex bias has yet to be determined. Finally, multiple environmental causes of ASD development are being considered due to their ability to influence sexes differently. As with the nature of multifactorial conditions, it is likely that many, if not all, of these mechanisms contribute to the development of ASD and have the possibility to impact males more directly than females. As with most areas of ASD research, more studies are needed to better understand this phenomenon.

\section{Animal models - sex differences in phenotypes}

Animal research in the field of neuroscience has predominately used male animals due to the assumption that cyclic female sex hormones may confound results (Cahill, 2012). A survey by Beery and Zucker (2009) reported a male bias in research across disciplines, with the ratio of male-only to female-only studies in neuroscience around 5:1 and no mention of subject sex in $22-42 \%$ of articles (Beery \& Zucker, 2011). New emphasis has recently been given for researchers to recognize the significance of considering sex and sex differences in the design and interpretation of studies ("Putting gender on the agenda," 2010), which provides much needed information about sex differences in animal models of diseases, like autism, that can be instrumental in advancing our understanding of the impact of sex on the human condition.

\subsection{Genetic}

The complexity of ASD etiology and known genetic component has led to the development of mouse models with targeted mutations of candidate genes (Oddi, Crusio, D'Amato, \& Pietropaolo, 2013). Recent advances in transgenic and recombinant 
technologies and the availability of the entire genome sequence allow genetic mouse models of autism to provide better opportunities to understand the genotype-phenotype relationship in autism (Bolivar, Walters, \& Phoenix, 2007). With targeted mutations in the mouse genome, researchers can test hypotheses about autism susceptibility genes and apply relevant assays to identify potential aspects of the autism behavioral, histological, or molecular phenotype (Crawley, 2007). Autism researchers are still developing model systems for evaluating hypotheses about the genetic causes of ASD (Roullet \& Crawley, 2011), and the work using these models to investigate potential sex differences is limited. Nevertheless, just as in the human condition, sex differences in phenotype have been described in genetic mouse models of autism.

Mouse models in autism research using mutations in the Fmrl gene are common because of the high proportion of individuals with FXS that also meet the diagnostic criteria for autism (25-40\%) (Roullet \& Crawley, 2011; Wassink et al., 2001) (see section 3.1 for further information on FXS and autism). Fmrl knockout mice display behaviors relevant to ASD, including hyperactivity, high anxiety-like behaviors, low prepulse inhibition (PPI) of acoustic startle, and slight impairment in maze learning (Roullet \& Crawley, 2011). Due to the sex differences in prevalence and phenotype in ASD, researchers have begun using animal models of FXS to investigate any sex differences in behavioral and physiological phenotype.

A study by Baker et al. (2010) explored spatial learning and memory in Fmrl knockout (KO) mice with C57 albino background (Baker et al., 2010). Fmrl KO mice displayed behavioral differences when compared to wildtype (Wt) littermates, including enhanced PPI and increased anxiety-like behaviors; however, there was no effect of sex 
on these behaviors (Baker et al., 2010). In other tests, such as platform test, trace fear conditioning task, and cued Morris water maze, both sexes of KO mice displayed normal performance (Baker et al., 2010). Another study evaluating sex differences in Fmrl KO mice produced similar findings using $\mathrm{C} 57 \mathrm{Bl} / 6$ as the background strain. Overall, Fmr I KO mice of both sexes exhibited audiogenic seizures, increased locomotor activity in the open field test, less freezing in contextual fear conditioning, impaired memory in passive avoidance examination, and increased PPI with low stimulus intensity. However, similar effects were seen in both sexes and there was no effect of sex on the Fmrl KO behavior (Ding, Sethna, \& Wang, 2014).

Behavioral testing has produced similar results in males and females of the Fmrl KO mouse model of FXS, yet other assays have yielded sex differences. In a study of expression of calcium-binding proteins in the dorsal thalamus of Fmrl KO mice and $\mathrm{Wt}$ littermates, males had a reduction in calbindin-immunoreactive cells in some dorsal thalamic nuclei compared to $\mathrm{Wt}$, while female $\mathrm{KO}$ mice did not show significant differences (Giraldez-Perez et al., 2013). No sex differences in calbindin immunoreactivity were observed in other areas of the brain, including the olfactory bulb, cerebral cortex, claustroamygdaloid region, striatum, or hypothalamus (Giraldez-Perez et al., 2013). As the stress response is often elevated in FXS, researchers sought to evaluate serum glucocorticoid levels in the mouse model of FXS (Fmrl KO). As is expected in rodents, female animals had higher serum corticosterone levels than males in unstressed conditions, and after an acute restraint, corticosterone levels increased in both sexes of KO and Wt mice (Markham et al., 2010). The increase was similar for females of both genotypes, but $\mathrm{KO}$ males showed an inhibited rise compared to $\mathrm{Wt}$; however, both sexes 
of Fmrl KO mice displayed a delayed return to baseline corticosterone levels following stress, which is similar to human FXS patients (Markham, Beckel-Mitchener, Estrada, \& Greenough, 2006).

Phosphatase and tensin homolog on chromosome ten (PTEN) is a known tumor suppressor gene with mutations associated with macrocephaly and autism; mice with Pten null mutations express macroencephaly, neuronal hypertrophy, problems with spatial learning, increased anxiety-like behaviors and activity, elevated acoustic startle and diminished social interests (Roullet \& Crawley, 2011). A study by Tilot et al. (2014) induced a Pten mutation in mice and found that only male Pten ${ }^{m 3 m 4 / m 3 m 4}$ mice exhibited decreased body size/weight compared to Wt littermates (Tilot et al., 2014). In a previous study the same research group demonstrated that brain weight of Pten ${ }^{m 3 m 4 / m 3 m 4}$ mice is double the brain weight of Wt animals, but there were not sex differences in brain growth of the mice with the Pten mutation (Mester, Tilot, Rybicki, Frazier, \& Eng, 2011; Tilot et al., 2014). Male Pten mutant animals displayed increased social preference compared to $\mathrm{Wt}$, while female mutant mice behaved the same as Wt (Tilot et al., 2014). There were no sex differences identified for other behavioral measures, such as novel object recognition, tail flick, open field, or rotarod measures (Tilot et al., 2014).

Another group evaluated brain size and social behavior in mice with haploinsufficiency for genes associated with increased susceptibility to ASD, PTEN and/or Serotonin transporter (SLC6A4) (Page, Kuti, Prestia, \& Sur, 2009). Pten functions to regulate the PI3-kinase (PI3K) pathway, and SLC6A4 codes for a membrane-bound protien that transports serotonin from the synaptic cleft to the presynaptic neuron; there is biochemical evidence that these pathways interact (Page et al., 2009). In animals with 
haploinsufficiency for Pten or Slc6a4, both sexes displayed a macrocephaly phenotype,

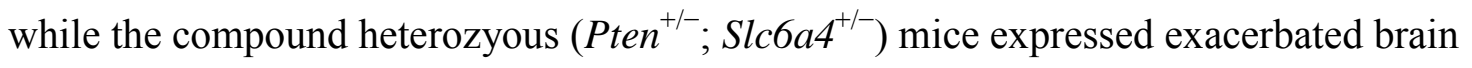
overgrowth more severe than either single haploinsufficiency (Page et al., 2009). Pten and Slc6a4 haploinsufficient female mice displayed social deficits compared with $\mathrm{Wt}$ with the compound heterozyous mice displaying the most impairment; however, no differences in social interaction were observed for any genotypes in the male groups (Pten haploinsufficient, Slc6a4 haploinsufficient, and $\operatorname{Pten}^{+/}$; Slc6a4 ${ }^{+/}$) (Page et al., 2009). Both sexes of Pten haploinsufficient and $\mathrm{Pten}^{+/-}$; Slc $6 a 4^{+/-}$mice had deficiencies in prepulse inhibition of the acoustic startle response as compared to Wt but not as compared to one another (Page et al., 2009).

Genetic mouse models of autism (or related conditions) are useful tools to better elucidate the genotype-phenotype relationship. To date, limited sex differences in behavior have been found in Fmrl KO mice; however, sexual dimorphisms in morphological and physiological traits have been identified in this model. Interestingly, the Pten mutation resulted in increased brain weight in both sexes, but smaller body weight in only males. In addition Pten mutated males displayed increased preference for social target (opposite of that seen in ASD), while only the females with Pten mutations displayed social deficits. More research is needed to further evaluate sex differences in these and other genetic models of autism.

\subsection{Valproic acid}

Valproic acid (aka 2-propylpentanoic acid, valproate, or VPA), used both to treat epilepsy and as a mood stabilizer in those with bipolar disorder (Roullet, Lai, \& Foster, 2013), is a known teratogen, with multiple pregnancy registries confirming an increased 
risk of major congenital malformations and correlations to reduced cognitive abilities following fetal exposure (Meador \& Loring, 2013). Recently, connections have been made between prenatal VPA exposure and ASD. In a population-based study of 655,615 children born in Denmark from 1996 to 2006, Christensen et al. (2013) found an increased risk of ASD (absolute risk, 4.42\%; adjusted hazard ratio, 2.9 [95\% CI, 1.7-4.9]) and childhood autism (absolute risk, 2.50\%; adjusted hazard ratio, 5.2 [95\% CI, 2.710.0]) among the 508 children exposed to VPA (Christensen et al., 2013). Given the connection between ASD and epilepsy (reported in approximately $1 / 3$ of autism cases) (Muhle et al., 2004; Tuchman, Cuccaro, \& Alessandri, 2010), it is noteworthy that the increased risk remained significant after adjusting for parental psychiatric disease and epilepsy (Christensen et al., 2013; Meador \& Loring, 2013).

Evidence from clinical studies indicating in utero exposure to VPA increases ASD risk motivated researchers to develop an animal model of ASD (Roullet et al., 2013). There is often uncertainty about the application of animal models to social/cognitive disorders and mental illness. However, numerous independent studies have successfully reported that the VPA animal model can be used to study the core symptoms of ASD; the literature supports both high face and construct validity for prenatal VPA treatment as an animal model of ASD (Roullet et al., 2013). Studies in both rats and mice confirm that prenatal VPA exposure leads to autistic-like behaviors in offspring, including social deficits, increased repetitive behaviors, and deficits in communication (Roullet et al., 2013). In addition, VPA has been shown to cause delayed physical development, reduced olfactory discrimination, and abnormal pre-weaning social behavior in mice (Roullet, Wollaston, Decatanzaro, \& Foster, 2010). VPA 
treatment in rodent models is also linked to reductions in Purkinje cell number and cerebellar volume (Ingram, Peckham, Tisdale, \& Rodier, 2000), decreased neuroligin 3 mRNA expression (Kolozsi, Mackenzie, Roullet, deCatanzaro, \& Foster, 2009), increased corticosterone and decreased immunological activity (Schneider et al., 2008), and lower cortical expression of brain-derived neurotrophic factor (BDNF) mRNA (Roullet et al., 2010), all of which are relevant to ASD.

As with ASD, there is evidence of sex differences in VPA exposure effects. Prenatal VPA treatment in rats caused long-term, sex-specific effects on postnatal behavior, including lower nociception reactivity, increased anxiety, and longer latency to social behaviors in VPA males compared to VPA females; however, differences in repetitive/stereotypic activity were similar in both sexes (Schneider et al., 2008). Mice also display sex-dependent morphological changes, with both sexes showing decreased Nissl-positive cell numbers in the prefrontal cortex, but only males displaying cell loss in the somatosensory cortex following VPA exposure (Hara et al., 2012). In a series of neurodevelopmental tests, mice exposed to VPA displayed timing differences in development compared to controls, with sex differences observed in mid-air righting, hanging wire grip strength, water maze, and motor activity (Wagner, Reuhl, Cheh, McRae, \& Halladay, 2006). In addition, mice exhibited social interaction deficits, anxiety-like behavior and memory deficits; however, the social interaction deficits were limited to male mice (Kataoka et al., 2013).

\subsection{Maternal stress}

Rodent models of maternal stress reveal how prenatal stress impacts the HPA axis by increasing glucocorticoid secretion leading to structural changes in the brain 
(Weinstock, 2008). We know from clinical studies that prenatal maternal stress is correlated with increased susceptibility to conditions such as ASD (Mueller \& Bale, 2007, 2008), see section 3.3 for more detail. One mechanism by which perinatal stress can cause sexually dimorphic responses is by altering the timing of HPG axis activation, which would affect male gonad development and subsequent testosterone production. Furthermore, numerous studies using rodent models also indicate increased male vulnerability to cognitive and behavioral deficits, many of which are relevant to autism.

In a study of maternal stress in rats, repeated variable prenatal stress during the third week of gestation produced deficits in object recognition, spatial reference, conditioned fear, and object discrimination memory (Markham et al., 2010). In addition, exposed male offspring were unable to gain competence on the novel object recognition task and their ability to extinguish conditioned responses to tone in the fear conditioning task was impaired, while prenatally stressed female rats were unaffected on these tasks (Markham et al., 2010). Chronic maternal stress during the second half of gestation and nursing in guinea pigs resulted in lower body weight and decreased activity in open field for male offspring compared to controls, but there was no effect on body weight or activity in female offspring; both males and females from stressed mothers, however, displayed elevated basal salivary cortisol postnatally (Emack, Kostaki, Walker, \& Matthews, 2008). Mice dams were exposed to chronic variable stress in early, mid, or late gestation; male offspring from dams exposed to early gestational stress showed significant impairments in learning performance, while females exposed during the same time period showed enhanced performance (Mueller \& Bale, 2007). Early postnatal stress resulting from chronic and unpredictable maternal separation (PD1-14) also produces sex 
differences; males reared under maternal stress displayed depressive-like behaviors in forced swim and tail suspension tasks (Franklin et al., 2010). Moreover, the separated male (but not female) pups had DNA methylation changes in the promotor regions of several germline genes, which were transmitted across several generations resulting in depressive behavior in offspring that were themselves reared normally (Franklin et al., 2010).

Studies evaluating sex differences in genetic models of autism have primarily failed to show sex differences in behavior, although differences in brain morphology and physiology between the sexes have been found. Further research in additional models involving targeted mutations of candidate genes is warranted. VPA and maternal stress rodent models have been evaluated more thoroughly for sex differences, and both have displayed more robust behavioral variations between the sexes, with VPA resulting in increased nociceptor reactivity, anxiety, and decreased social behavior in males only, and maternal stress inducing impairments in novel object recognition, fear conditioning, learning performance, and increased depressive behaviors in males (but not females). Both of these models strongly indicate increased male vulnerability to environmental insults, producing cognitive and behavioral deficits such as increased anxiety, decreased sociability, and increased depressive behaviors, which are relevant to ASD. Mechanisms that increase male susceptibility in these areas appear to be conserved from rodents to humans. These models provide a promising area of research to better understand the mechanisms that contribute to this sex bias in vulnerability and its relevance to ASD. 


\section{Conclusions}

This review has compiled data from both clinical studies and animal models of ASD with a focus on the sex bias and underscores the importance of ending the overrepresentation of males in autism research. While it is possible that current diagnostic criteria fail to detect ASD in some high-functioning girls or that ascertainment bias slightly overestimates the bias, the available evidence strongly indicates a sex difference in ASD prevalence. In addition, clinical and basic research has found morphological, physiological, and behavioral differences in the manifestation of the ASD phenotype between the sexes. This body of work highlights the lingering uncertainty regarding the mechanism responsible for this sex bias. Just as ASD is proposed to result from multiple, variable factors, the sex bias in autism is likely the result of more than one factor that either increases the vulnerability of males or protects females. Sex chromosomes, parental imprinting, hormone exposure (gonadal or neurosteroidogenic), and environmental mechanisms, such as increased parental age or maternal stress or immune response, are current targets for further research. While animal models have limitations in regards to human ASD pathology, they present valuable means to better understand the relationship between genotype and phenotype and to evaluate aspects of environmental exposures. Although recent work has shed light onto the exploration of the sex differences in ASD, the collection of research presented here emphasizes the need to better understand the mechanism(s) responsible for the sex differences.

\section{Organization of Dissertation}

This dissertation is focused on understanding the interaction of biological sex, perinatal testosterone exposure, and behavioral and morphological pathologies associated 
with autism using a mouse model. A common theme in autism research is the multiple hit hypothesis. Casanova (2007) proposed that ASD etiology includes (1) inheritance of genetic susceptibility and (2) an environmental agent or endogenous stressor that (3) occurs during a critical time period in brain development (Casanova, 2007), also known as the triple hit hypothesis. Another 3-hit theory for contributions to ASD, developed by Davis and Pfaff (2014), includes androgenic hormone effects as one of the hits (Davis \& Pfaff, 2014). As ASD is proposed to result from multiple, variable factors, the sex bias in autism is likely the result of more than one factor that either increases the vulnerability of males or protects females. In chapter two of this dissertation I establish baseline data regarding sex differences in a wheel running assay designed to measure autism-like behaviors, such as habit formation, cognitive rigidity, stereotypical behaviors, and social interaction in 3 commonly used strains of developmentally normal mice. Chapter three focuses on the effects of perinatal testosterone on behavior and brain morphology in a mouse model of autism. Specifically, my work used a mouse model of autism to mimic differential susceptibility of males and females to a prenatal insult, VPA, and to determine whether masculinizing females perinatally with testosterone (T) following VPA treatment will also masculinize their risk for abnormalities in brain development and behavior relevant to ASD. Chapter four is a summary of the findings from my dissertation research with possible future directions presented. 


\section{CHAPTER 2}

\section{INVESTIGATION OF PRE-PUBERTAL SEX DIFFERENCES IN WHEEL RUNNING}

\section{AND SOCIAL BEHAVIOR IN THREE MOUSE STRAINS}

\section{Introduction}

To date, neuroscience research remains biased towards male animals, following the assumption that cyclic sex hormones in females may confound results (Cahill, 2012). A 2009 survey showed a male bias across research disciplines, with the ratio of maleonly to female-only studies in neuroscience at 5:1 and subject sex omitted in $22-42 \%$ of articles (Beery \& Zucker, 2011). More disturbingly, investigators often assert that findings from male-only studies lead to conclusions that are applied to both sexes (Cahill, 2012). Researchers across disciplines now recognize the importance of considering sex and sex differences in the design and interpretation of studies ("Putting gender on the agenda," 2010). Recent suggestions include incorporating females and males in equal numbers with a clear comparison of the two sexes (Prendergast, Onishi, \& Zucker, 2014).

The classic Organizational-Activational Hypothesis proposed by Phoenix et al.(1959), which states that gonadal hormones activate brain regions in adulthood previously organized by those hormones early in development, has been expanded to more accurately represent the complexities of sexual development. Sex differences in the brain and behavior result from intricate interactions among steroid hormones synthesized in both the gonads and the brain; sex chromosomes acting at the genetic, cellular, and biochemical level; and the environment from early development throughout the lifespan 
(Lenz et al., 2012; Prendergast et al., 2014). Sex differences in the brain occur at many levels, including differences in regional volume and/or cell number, morphology, physiology, molecular signaling, and gene expression (Lenz et al., 2012), leading to differences in learning and memory, fear, anxiety, and nociception (McCarthy et al., 2012; Prendergast et al., 2014).

Mammalian species tend to exhibit sex differences in social behavior as adults, especially those relating to mating and courtship; however, differences often extend beyond obvious variations in mating behavior (Meaney, Stewart, \& Beatty, 1985). Previous studies have reported no apparent sex-related differences in general activity in the mouse (Lamberty \& Gower, 1988), nor an effect of estrous cycle on activity in adult mice (Dowse, Umemori, \& Koide, 2010; Meziane, Ouagazzal, Aubert, Wietrzych, \& Krezel, 2007). However, other evidence has linked sex steroids to wheel running distance, speed, and duration in both sexes of adult mice (Bowen et al., 2012) and shown that in the Hsd:ICR strain (outbred descendants of the Swiss-Webster strain), females spend more time wheel running and at a faster speed than males (Swallow, Carter, \& Garland, 1998). These measures did not differ by sex in lines of mice selectively bred from the Hsd:ICR strain for high wheel running activity (Garland et al., 2011).

In addition, many mammalian species display sex differences in social play of juveniles. Generally, male rats are more likely to initiate and engage in play-fighting behaviors than females, whereas females are more likely to withdraw from a play initiation (Meaney et al., 1985). Although mice do not generally display 'rough and tumble play,' other measures to assess social reciprocity and play include nose-to-nose sniffing, anogenital sniffing, following, crawling over/under, and social grooming 
(Crawley, 2007). Unlike the mating/courtship behavioral differences seen in adult animals, these social behaviors are seen pre-pubertally, i.e., before sexual maturity. Any disparities seen in behavior at this stage are more likely due to the perinatal environment, sex chromosomes or other prenatal effects than to differences in gonadal hormones circulating at the time of the behavioral trial.

In order to effectively model behavioral disorders using animals of both sexes, it is important to assess baseline sex-related behavioral differences, especially when studying disorders for which sex-related behavioral effects are expected, such as autism. The sex bias of autism has a male to female ratio of 4:1 and even higher in cases without dysmorphic features and Asperger syndrome (Moldin \& Rubenstein, 2006). Recent research has stimulated the generation of animal models to better elucidate the role of specific genes and environmental influences in the pathogenesis of autism (Oddi et al., 2013).

Current neuroscience literature supports a variety of mouse behavioral assays designed to maximize relevance to social deficits specific to autism (Silverman, Yang, Lord, \& Crawley, 2010). The results from previous studies using mouse models of autism have underscored the importance of including age and sex as relevant factors (Oddi et al., 2013). The strains chosen for this study were inbred (C57BL/6) and outbred (CFW and CF1) mice, commonly used as general multipurpose models. On postnatal days 27-33, we investigated the effect of sex on distinct behaviors. Using a series of running wheel assays developed to evaluate autism-like behaviors (Karvat \& Kimchi, 2012), we measured the animals' ability to gain and maintain a routine, assessed repetitive behavior, evaluated cognitive rigidity and examined social interactions. This behavioral array was 
chosen because of its relevance to the core symptoms of autism and in preparation for future work assessing autism-like behaviors following developmental insults. Examining the animals before puberty should reduce behavioral variations due to circulating sex hormones. By assessing three mouse strains, we attempted to find information that applies more generally to the species, rather than to only one strain. Most importantly, these assays provide needed insight into pre-pubertal sex-related differences in behavior.

\section{Materials and Methods}

\subsection{Animals}

The animals used in this study were 3 strains of mice: C57BL/6 ( $n=16 ; 8$ males and 8 females), CFW ( $n=24 ; 12$ males and 12 females), and CF1 ( $n=20 ; 10$ males and 10 females). The mice were received from the vendor (C57BL/6, Jackson Laboratory; CFW and CF1, Charles River) at approximately 3 weeks of age (P20-P21). Initial body weights were taken on the day of arrival, which was P20 for C57BL/6 and P21 for CFW and CF1. All animals were maintained in our animal facility on a 12:12 light/dark cycle and were provided with TekLad 2016 rodent chow and tap water ad lib. The C57BL/6 mice remained on their previous light schedule (lights off 6pm), while the CFW and CF1 mice underwent a phase shift to correspond with the researchers' schedule. On day one lights went off at $6 \mathrm{pm}$. On day 2 the mice underwent a 3 hour phase shift, with lights off at $3 \mathrm{pm}$. The CFW mice underwent another 3 hour shift on day 3 resulting in lights off at $12 \mathrm{pm}$ (note that daylight savings time started immediately after this shift so that the lights actually went off at $1 \mathrm{pm}$ during trials), whereas the CF1 mice underwent a 2 hour shift on day 3 resulting in lights off at $1 \mathrm{pm}$. The light schedule on subsequent days was maintained. Within each strain, two mice of each sex were housed separately to be used 
for social testing purposes only; in CFW and CF1 strains these mice were also shipped separately. After a week-long acclimation period, the experimental animals were tested with a series of wheel-running assays (see section 2.2). Euthanasia was performed following a standard protocol of intracardial perfusion with $4 \%$ paraformaldehyde; final body weights, testis and uterine weights were taken at this time to assess growth and sexual maturity. The C57BL/6 mice of both sexes were perfused in two groups on postnatal days 34 and 35. All CFW and CF1 females were perfused on postnatal day 33, while all males were perfused on postnatal day 34 . Animal care provided was in accordance with the Guide for the Care and Use of Laboratory Animals (Guide for the Care and Use of Laboratory Animals, 2011) and procedures were approved by our Institutional Animal Care and Use Committee. Note: The trials for each strain were completed independently so that only one strain of mice was housed and tested at a time.

\subsection{Test Procedure}

Animals were tested during the dark phase of the light cycle to ensure they were in their most active period when tested. Video was collected using a camera with nightvision capability under red lights. The wheel-running assays used a plexiglass cage (34.5 $\mathrm{cm} \times 23 \mathrm{~cm}$ x $19 \mathrm{~cm}$, covered) lined with corncob bedding and fitted with a standard plastic mouse running wheel (14 cm diameter) that either freely turned or was jammed by a metal pin. To remove olfactory cues, the layer of bedding was changed and the entire cage was cleaned with $70 \%$ ethanol between trials. We followed the protocol of Karvat \& Kimchi (2012) to assess wheel running behaviors. Modifications were made to assess sex differences in behavior, as specified subsequently. Stage 1: gaining and maintaining a routine. For the $\mathrm{C} 57 \mathrm{BL} / 6$ strain each mouse was placed in the cage for 20 minutes and 
permitted to run on the wheel freely for 4 consecutive days; the trial time was reduced to 15 minutes and period reduced to 3 consecutive days for CFW and CF 1 strains, as analysis of C57BL/6 data revealed this timing should be sufficient for routine wheel running to be established. Stage 2: repetitive behavior. Each mouse was recorded for 15 minutes in the cage with the wheel jammed on days 5-6 (for C57BL/6) or days 4-5 (CFW and CF1). For each sex, time spent grooming and burrowing in the bedding on days the wheel was free (1-4 or 1-3) and on days the wheel was jammed (5-6 or 4-5) was assessed. Stage 3: cognitive rigidity. Cognitive rigidity is defined as the inability to forgo a habit/routine. Time spent interacting with the wheel when it was jammed was measured and evaluated for sex-related differences in cognitive rigidity. Stage 4: social interaction. On day 7 (C57BL/6) or day 6 (CFW and CF1) each study mouse spent 10 minutes in the cage with the jammed wheel and an age and sex-matched novel mouse. The novel mouse was placed into the cage first, followed by the study mouse to allow the observer to identify the mice and score behaviors accordingly. We tested for social interaction by assessing the time spent by the study mouse engaging in social investigation with a novel mouse. Contact and following behaviors were assessed for differences in social behavior between the sexes.

\subsection{Scoring}

Behavior scores were recorded using the Noldus Observer software version 4.1. For all days, the following items were measured: latency to begin wheel running; directionality and duration of wheel running; interaction with wheel without movement (i.e., sitting on wheel), and duration of burrowing, grooming, and interaction with the cage's water nozzle. For durations, percentage of total trial was used instead of time, 
because the total time differed among strains. Burrowing was scored when pieces of the bedding were intentionally moved by the mouse. On the days the wheel was jammed, "wheel running" was scored as interaction of the mouse with the wheel that would have made the wheel move if it were free. Time the mouse spent on top of the jammed wheel was excluded from the wheel interaction score. On the final day, interaction of the study mouse and a novel, age and sex-matched mouse provided information on sociability. Any physical engagement between the mice, whether initiated by the study mouse or by the novel mouse, was scored as social interaction, divided into contact and following behaviors.

\subsection{Statistics}

Running wheel assays (i.e., latency and duration of wheel running and stereotypical behaviors) were analyzed for sex, day and sex $\mathrm{x}$ day interactions in SAS (9.3, SAS Institute Inc.) using a mixed linear model with the restricted/residual maximum likelihood (REML) estimation method. The Kenward-Roger approach was used to approximate inference about fixed effects to estimate denominator degrees of freedom in tests for fixed effects. Day and sex x day were specified as random effects. Data were transformed as appropriate to reach normality: for C57BL/6 data the square root was taken for the duration of wheel running and interaction with the water nozzle, and for the latency to wheel run; for CFW the duration of wheel running data was squared, the square root was taken for duration grooming data and the natural log was taken for duration of interaction with the water nozzle; for CF1 the duration of wheel running data was squared, while the square root was taken for latency to wheel run. To adjust for the number of simultaneous tests to assess the effect of day, the sequential Bonferroni test 
was used (Rice, 1988). The latency and duration of contact and following behaviors of the study mouse with a novel mouse were analyzed by t-test with sex as an independent variable. Strain differences in wheel running duration was analyzed by one-way ANOVA with pairwise multiple comparisons made using the Holm-Sidak method. Because we found no significant sex differences within each strain, this analysis combined data for all mice of a given strain.

\section{Results}

In all 3 strains (C57BL/6, CFW and CF1), pre-pubertal mice follow patterns of wheel running behavior characteristic of normal adult mice, with some differences among strains; however, there are not significant differences in wheel running behaviors between the sexes at this age.

\subsection{Latency of Behavior}

In a typical animal gaining a routine, latency to run should decrease following the initial introduction to the novel wheel. The mixed model analysis for latency to begin wheel running revealed a significant effect of day for analyses of days 1-6 for C57BL/6 $(\mathrm{p}<0.0001)$ and days $1-5$ for $\mathrm{CFW}(\mathrm{p}<0.0001)$ and $\mathrm{CF} 1(\mathrm{p}=0.0002)($ Fig $1, A-\mathrm{C})$. For days the wheel was free, latency to run was significantly less on day 2 compared to day 1 across all strains $(\mathrm{p}<0.0001)$, displaying appropriate routine gaining. Cognitive rigidity is evaluated by the inability to forgo a routine. Latency to run increased on the second day the wheel was jammed as compared to the first $(\mathrm{p}<0.001$ for all strains), suggesting that the mice in our study remembered the wheel was jammed the previous day and could adjust their routine. We found no significant difference in latency to run on the wheel between males and females across days for C57BL/6, CFW, or CF1 $(p=0.3304$, 
$\mathrm{p}=0.9399, \mathrm{p}=0.5988$, respectively). The observer noted that $\mathrm{C} 57 \mathrm{BL} / 6$ males were more inclined to explore the cage on day one and appeared to initiate wheel running later than C57BL/6 females. The mean latency to run on the wheel for females was $159.8 \pm 11.2$ seconds and for males was $238.8 \pm 40.0$ seconds; however, the latency data across all days failed to reach statistical significance for $\operatorname{sex}(\mathrm{p}=0.3304)$ and there was no significant sex $\mathrm{x}$ day interaction $(\mathrm{p}=0.4062)$. Social behavior was demonstrated by the latency of the study mouse to initiate contact or begin following the novel mouse. For all strains, t-tests indicated there was no difference between sexes in latency to contact or follow the novel mouse (Fig 2, A-B) ( $\mathrm{p}=0.134$ to $\mathrm{p}=0.942)$.

\subsection{Duration of Behavior}

The percentage of trial duration spent wheel running was also analyzed using a mixed effects model in SAS (Fig 1, D-F). Duration analysis again revealed a significant day effect during days 1-6 for C57BL/6 $(\mathrm{p}<0.0001)$ and days $1-5$ for CFW $(\mathrm{p}<0.0001)$ and CF1 ( $\mathrm{p}<0.0001)$. Total duration of wheel running increased across days the wheel was free; however, interaction with the wheel dropped for days when the wheel was jammed. In a comparison of wheel running duration on day 1 (first day free) vs. day $4 / 3$ (last day free) and also day 5/4 (first day jammed) vs. day 6/5 (second day jammed), all $\mathrm{p}$-values reached significance ( $\mathrm{p}<0.0003$ for all comparisons) using the Bonferroni adjustment, except CFW day 1 vs. day 3. This follows the expected pattern of behavior for animals lacking rigidity to change habits, i.e., a normal mouse should decrease its interaction time with the wheel on the second day that the wheel is jammed, which was apparent on day $6 / 5$. We found no significant difference in percentage duration of wheel running between the sexes across days for C57BL/6, CFW, or CF1 ( $\mathrm{p}=0.6146, \mathrm{p}=0.1674$, 
$\mathrm{p}=0.4881$, respectively). However, there were strain differences in percentage duration of wheel running for the last day the wheel was free $(\mathrm{p}<0.001)$, first day the wheel was jammed $(\mathrm{p}=0.001)$, and second day the wheel was jammed $(\mathrm{p}<0.001)$ (Table 1$)$. Total duration of contact and following behaviors with the novel mouse were examined from the social interaction test (Fig 2, C-D). The t-tests indicated no significant differences between sexes in contact or following behaviors of the study mouse with a novel mouse $(\mathrm{p}=0.114$ to $\mathrm{p}=0.866)$

We also evaluated the total duration percentage of stereotypical behaviors, such as burrowing, grooming, and interaction with the water nozzle. The ability to freely run on a wheel reduces stereotypical behaviors, e.g., burrowing in bedding (Karvat \& Kimchi, 2012). Therefore, a typical animal should spend more time performing stereotypical behaviors when the wheel is jammed vs. free. We compared these behaviors for the last day the wheel was free (day $4 / 3$ ) vs. the first day it was jammed (day $5 / 4$ ), and found that grooming and water nozzle interaction were displayed more often when the wheel was jammed ( $\mathrm{p}<0.0003$ for all strains). The inability to run on a wheel increased these stereotypical behaviors in both sexes. A day effect was noted for grooming behaviors in both CFW and CF1 strains (CFW p <0.0001, CF1 p=0.0042) (Fig 3, E-F). Statistical analysis of grooming in C57BL/6 mice was hindered by its infrequency in this strain, yet these animals displayed the same pattern of behavior as seen in other strains (Fig 3, D). A day effect of interaction with the water nozzle was displayed across all days ( $<<0.0001$ for all strains) (Fig 3, G-I). Burrowing was exceedingly rare while the wheel was free and represented a small percentage of time spent on days the wheel was jammed (Fig 3, A-C; Fig 4). A sex effect was seen for water nozzle interaction in CF1 mice across all days, in 
that males were more likely to interact with the nozzle than females $(p=0.0263)$, but not in any other strain $(p=0.3143$ and $p=0.5966)$. In addition, brief mutual grooming was displayed in both sexes during the social interaction trial. Both sexes displayed similar patterns of behavior in that they were more likely to interact with the wheel when it was free and performed other behaviors when it was jammed. See Figure 4 for a representation of total duration of all behaviors by sex and across days. Three days are shown for each sex from all strains: the last day the wheel was free (left column) and the subsequent two days when the wheel was jammed. This figure clearly displays our main findings: within each strain there was a lack of sex differences in behavior; however, strain differences in wheel running behavior were apparent (analysis in Table 1).

\subsection{Morphology}

Mouse morphology data, including body and gonad/uterine weight, can be found in Fig 5. The weights of both CFW and CF1 mice matched expected growth curves (Charles River Laboratories International Inc., 2014a, 2014b); C57BL/6 mice were above the expected weight at 3 weeks, but matched published growth curve weights by 5 weeks (The Jackson Laboratory, 2014).

\section{Discussion}

Wheel running is widely studied in rodents (Novak, 2012), as it appears to be a highly rewarding activity (Werme et al., 2002). Karvat and Kimchi (2012) used this instinctive wheel running propensity in mice to develop a behavioral test relevant to autism-like behaviors. It assesses key features including rigidity to change habits, repetitive stereotypical behaviors and impaired social interactions. We used this technique to evaluate sex differences in these behaviors in pre-pubertal mice. Overall, we 
found no significant sex differences in wheel running behaviors in 3 strains of prepubertal mice.

In adult rats, assessments of wheel running have repeatedly shown higher levels of activity in females (Beatty, 1992), and also shown that wheel running activity is variable along the estrous cycle (Kent, Hurd, \& Satinoff, 1991). Some studies of general activity and wheel running in adult mice have reported sex differences (Broida $\&$ Svare, 1984; Garland et al., 2011; Swallow et al., 1998), while others have not (Lamberty \& Gower, 1988). Sex steroids do appear to influence wheel running in adult C57BL/6 mice, in which females exhibit greater activity (i.e., distance and duration) than males at baseline; gonadectomy significantly reduced levels of wheel running (distance, duration, and velocity) in both males and females, which was at least partially recovered upon steroid replacement (Bowen et al., 2012). In addition, control Hsd:ICR mice showed sex differences in wheel running measures, including minutes per day and revolutions per day, and sex differences approached significance in average velocity (Garland et al., 2011). However, another study using C57BL/6 found no effect of estrous cycle on general activity in the open field assay (Meziane et al, 2007). With no previous data on pre-pubertal wheel running in mice, we explored the possibility of sex differences, which could result from differences in neural structure/circuitry of the sexes established perinatally. The similarity in wheel running behavior provides further evidence that wheel running behaviors may be linked to circulating sex steroids and differences only emerge after hormone levels increase following puberty. On the other hand, it is also plausible that a pre-pubertal sex difference in this behavior exists, but the sensitivity of the behavioral test may be inadequate to detect a difference between males and females 
(Kelly, Ostrowski, \& Wilson, 1999). It is worth noting that two of the strains underwent photoperiod phase shifting in the week before testing; however, this did not have noticeable effects on behavior in this assay, which was done in the dark phase for all strains.

We also found a lack of variability in social behaviors between the sexes. There were no significant sex differences in contact or following behaviors of the study mice with the novel mice. While it should be noted the study and novel mice used in the C57BL/6 strain were shipped in the same container prior to being separated for 13 days before the social interaction test, the study and novel mice in the other strains were shipped and housed separately to maintain novelty. Similar results were found across all strains for both contact and following behaviors. Social grooming, self-grooming, noseto-nose sniffing, ano-genital sniffing, following and exploratory activity were exhibited by both sexes.

Another factor that was inherent to our study is the timing of puberty. Since the mice were not reared onsite, they were received following weaning around 3 weeks of age. Mice were given a week-long acclimation period, followed by the 6-7 day wheelrunning assay. Perfusions were then performed between postnatal days 33 and 35 . Puberty is the transitional period prior to adulthood that terminates with the production of mature gametes and the start of reproductive activity (Mayer et al., 2010). Sexual maturity is usually reached in females with the onset of estrus between 40-51 days, depending on weight and other environmental conditions (Pinter, 2007). Uterine weight and morphology are standard measures of estrogen action in immature female rodents (Evans, 1941). The timing of puberty in males is suggested to begin around day 40 
(Albert, 1984). Considering the age of the animals, we can be relatively confident that our study was conducted before either sex reached sexual maturity. The variability in uterus weights suggests that some females might have been further along in puberty than others (Fig 5, C). Puberty occurs over time with the onset of neuroendocrine events that stimulate the pulsatile release of $\mathrm{GnRH}$, which activates the pituitary/gonadal axis and directs gonadal steroid hormone production and the maturation of gametes (Colledge, Mei, \& d'Anglemont de Tassigny, 2010; Mayer et al., 2010). Considering that increased gonadal hormone activity begins prior to sexual maturity at the conclusion of puberty, we cannot be certain that all animals were tested before the initiation of increased gonadotropic activity. However, with behavioral trials completed prior to P33 the influence of circulating gonadal hormones should have been minimal.

Both sexes showed characteristic wheel running behavior, spending the majority of each trial interacting with the wheel when it was free and more time performing other activities (e.g., stereotypical behaviors, general locomotion) when it was jammed. Given that sex differences were not detected in this assay, the results show that baseline prepubertal sex-related differences are not strong enough to influence behavior in runningwheel studies. Furthermore, the inclusion of three strains provides evidence that these results apply more generally to the species, rather than to only one strain. We found differences among strains, including duration of time spent wheel running (or attempting to run) when it is both free and jammed. Interestingly, sex differences in wheel running have been found in adult mice related to two of our strains (C57BL/6: Bowen et al, 1992; CFW and Hsd:ICR both descend from same Swiss-Webster stock: Swallow, Carter, \& Garland, 1998), but we found no sex differences within any of our tested strains before 
sexual maturity. We conclude that sex-related behavioral differences can be discounted when designing future experiments to measure the effects of developmental insults on wheel running behavior in juvenile mice. Therefore, if sex differences in the runningwheel assay emerge after a developmental insult, one can make inferences regarding relative susceptibility of males vs. females to that treatment. 
Table 1: Strain differences in duration of wheel running (mean $\% \pm$ SEM) on last day free (Last Free), first day jammed (Jammed 1), and second day jammed (Jammed 2).

\begin{tabular}{lccc}
\hline Strain & Last Free & Jammed 1 & Jammed 2 \\
\hline C57BL/6 & $69.9 \pm 2.96^{\mathbf{a}}$ & $16.6 \pm 0.73^{\mathbf{b}}$ & $4.9 \pm 0.48^{\mathbf{a}}$ \\
CFW & $55.0 \pm 1.86^{\mathbf{b}}$ & $13.6 \pm 0.63^{\mathbf{a}}$ & $2.3 \pm 0.52^{\mathrm{b}}$ \\
$\mathrm{CF} 1$ & $69.0 \pm 2.71^{\mathrm{a}}$ & $12.1 \pm 0.84^{\mathbf{a}}$ & $7.7 \pm 0.50^{\mathbf{c}}$ \\
\hline
\end{tabular}

${ }^{\text {abc }}$ Differing superscripts in a given column indicate statistically significant difference ( $p$ $<0.05$ ) across strains in $\%$ duration wheel running (or attempt to run in wheel when jammed) for that column. Males and females did not differ within strains for this measure (Fig 1, D-F), so are both included in this analysis. 

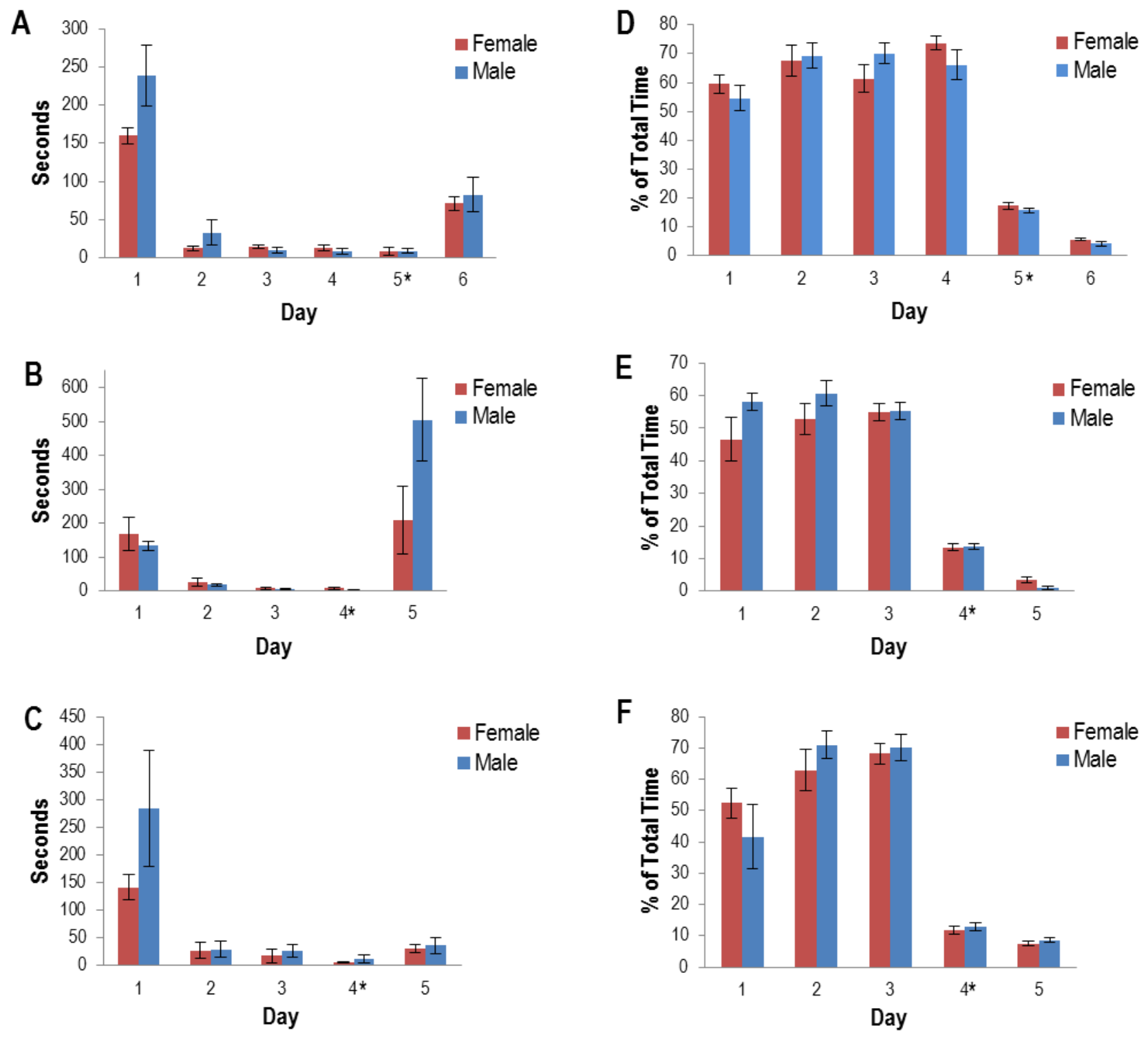

Fig. 1. Wheel running behavior. (A-C) Latency of mice to run on wheel (mean $\sec \pm$ SEM). A) C57BL/6, B) CFW, C) CF1; (D-F) Duration of wheel running (mean $\%$ total trial $\pm \mathrm{SEM}$ ). D) C57BL/6, E) CFW, F) CF1 * = first day the wheel was jammed. When the wheel is jammed, "wheel running" is defined as any activity that would make the wheel move if it were free. 

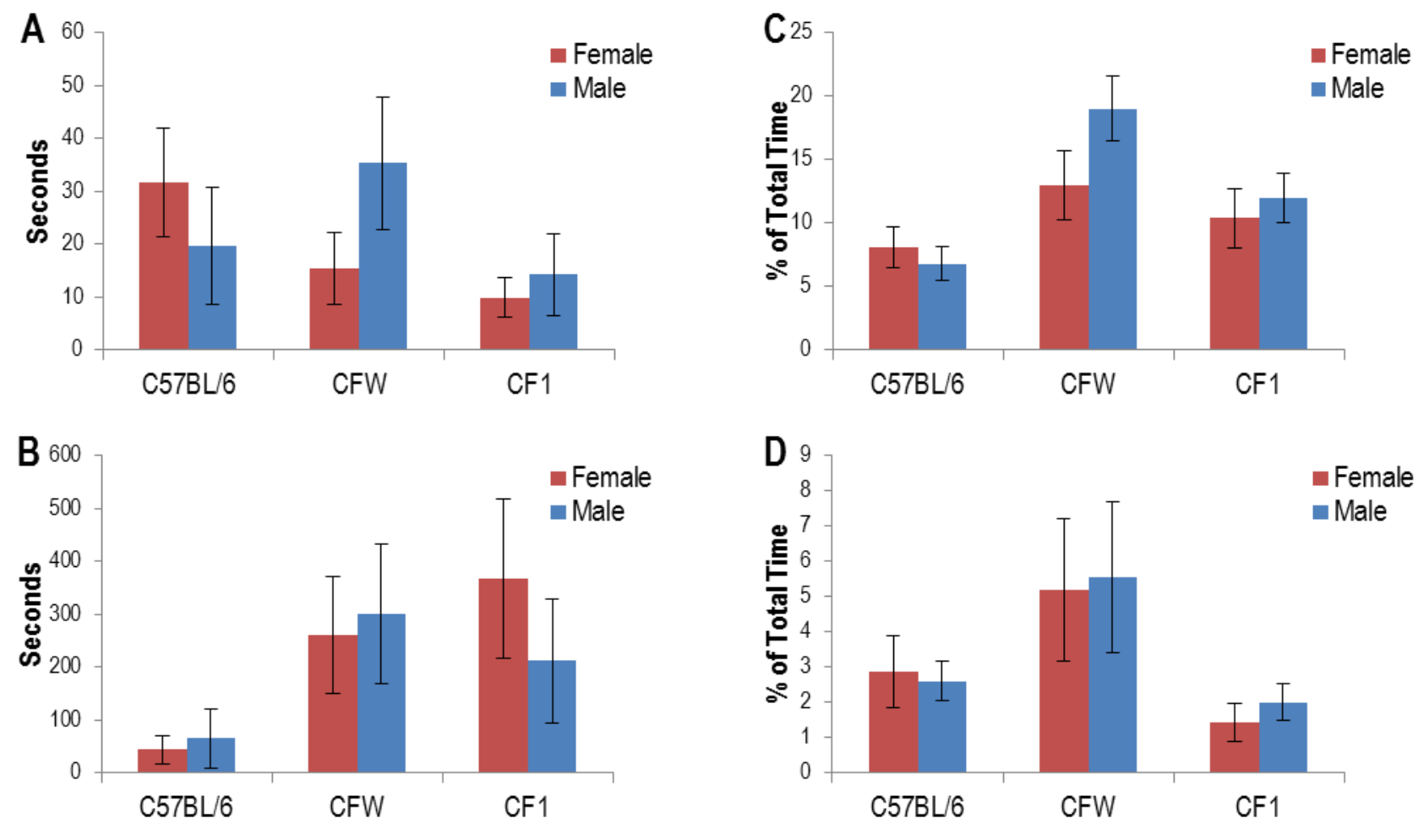

Fig. 2. Social behaviors. (A-B) Latency of social interaction with novel mouse (mean sec \pm SEM), analyzed for sex differences within strains. A) contact, C57BL/6, $p=0.215$; $\mathrm{CFW}, \mathrm{p}=0.134 ; \mathrm{CF} 1, \mathrm{p}=0.447 . \mathrm{B})$ follow, $\mathrm{C} 57 \mathrm{BL} / 6, \mathrm{p}=0.765 ; \mathrm{CFW}, \mathrm{p}=0.942 ; \mathrm{CF} 1$, $\mathrm{p}=0.595$. (C-D) duration of social interaction with novel mouse (mean $\%$ total trial \pm SEM). C) contact, C57BL/6, $\mathrm{p}=0.548 ; \mathrm{CFW}, \mathrm{p}=0.114$; $\mathrm{CF} 1, \mathrm{p}=0.495$. D) follow, C57BL/6, $\mathrm{p}=0.817 ; \mathrm{CFW}, \mathrm{p}=0.770 ; \mathrm{CF} 1, \mathrm{p}=0.866$. 
A

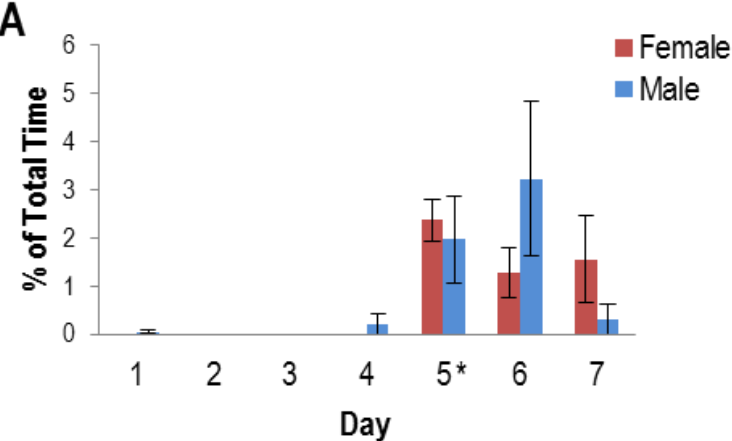

C

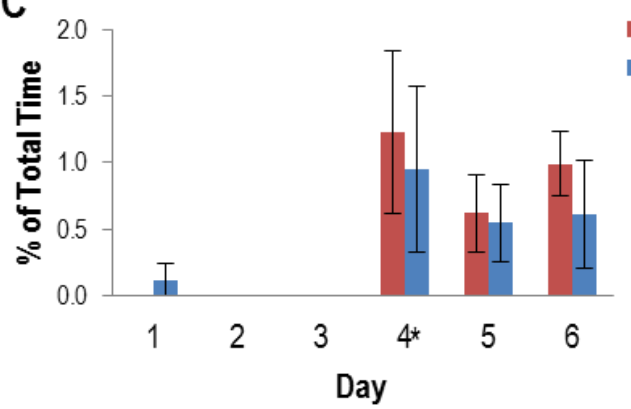

E
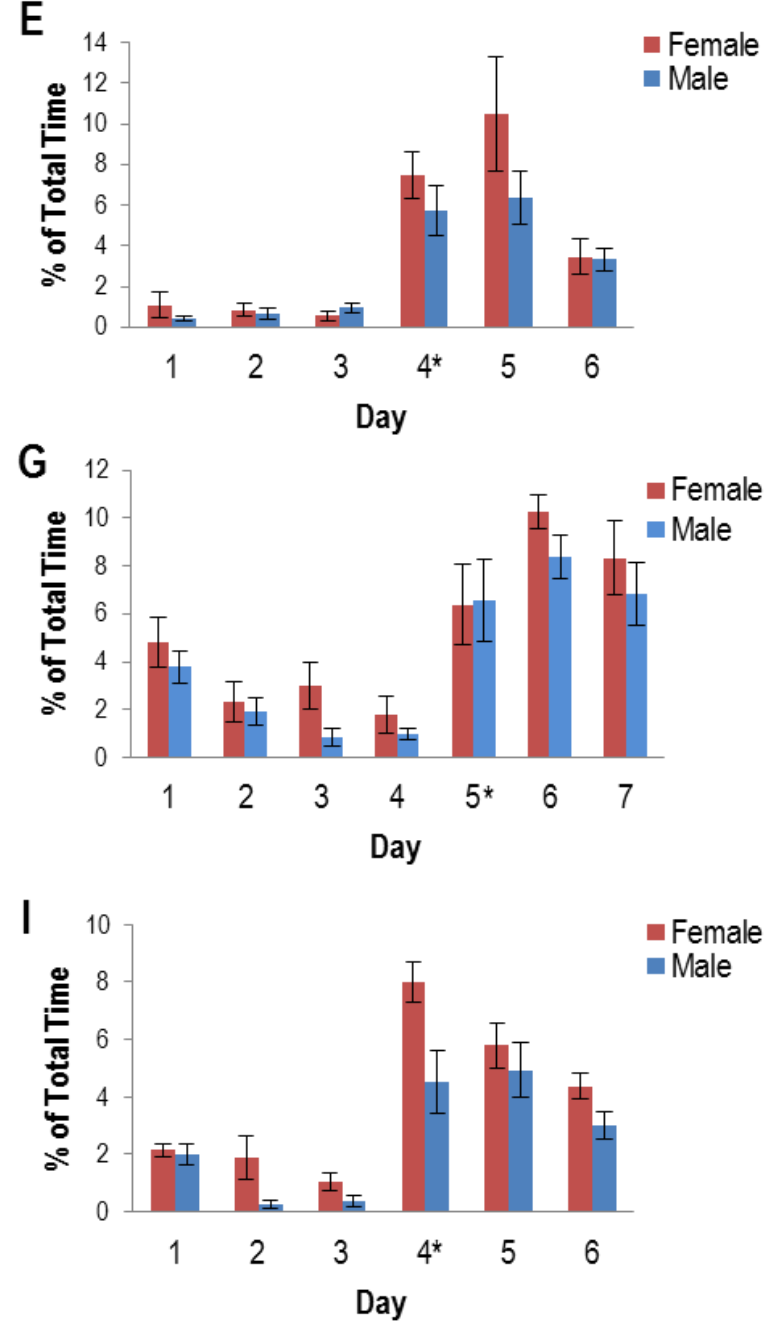

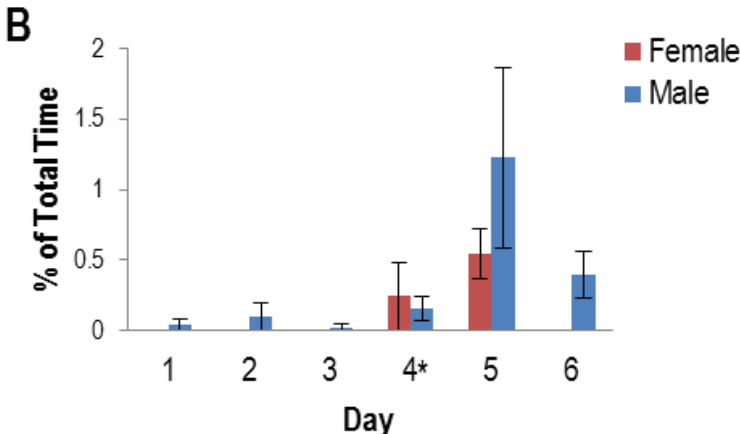

D

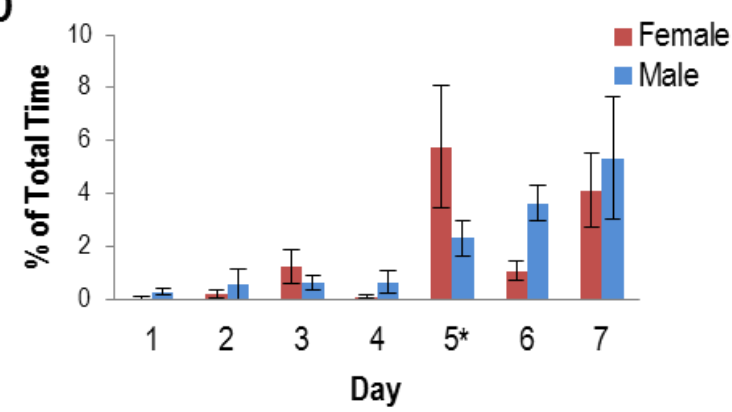

F
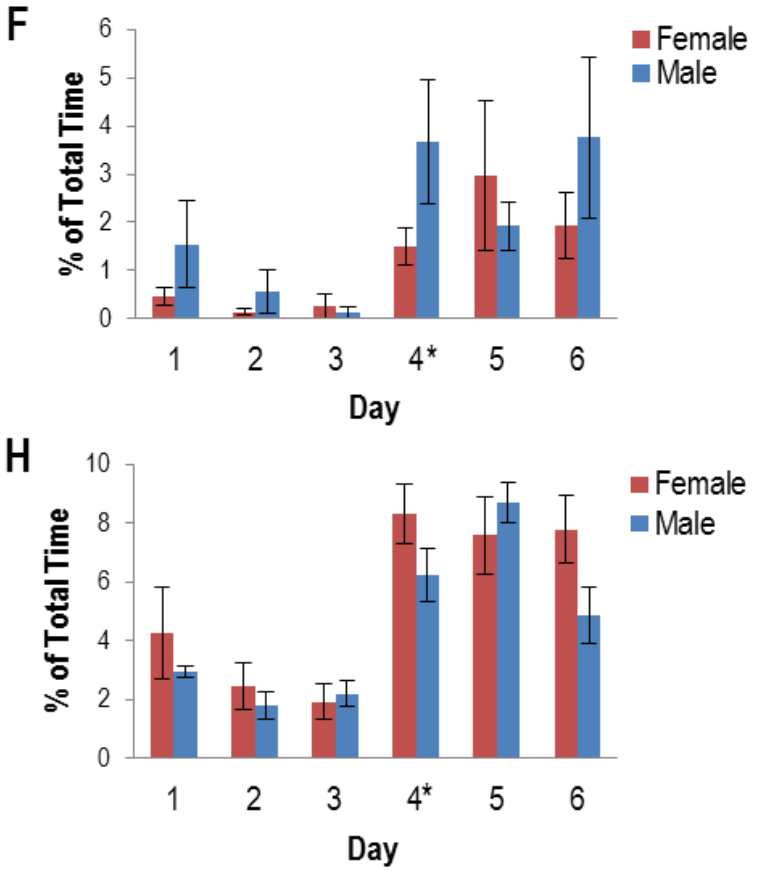
Fig. 3. Duration of sterotypical behaviors (mean $\% \pm$ SEM). (A-C) burrowing: A) C57BL/6, B) CFW, C) CF1; (D-F) grooming: D) C57BL/6, E) CFW, F) CF1; (G-I) water nozzle interaction: G) C57BL/6, H) CFW, I) CF1. * = the first day the wheel was jammed. 
Wheel free

$\mathrm{C} 57 \mathrm{BL} / 6$ female

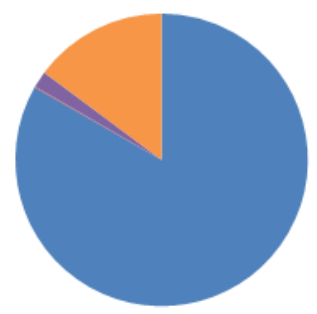

C57BL/6 male

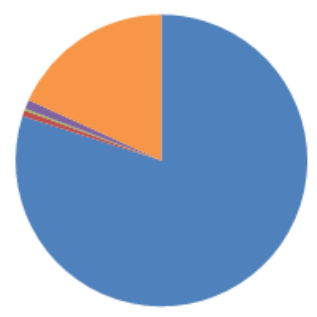

CFW female

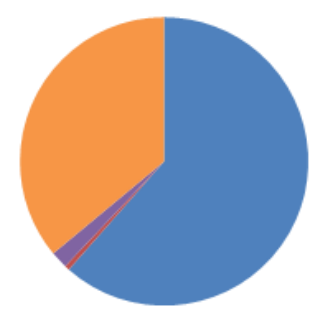

CFW male

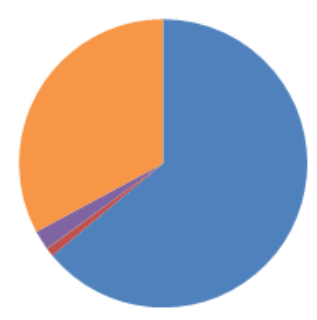

CF1 female

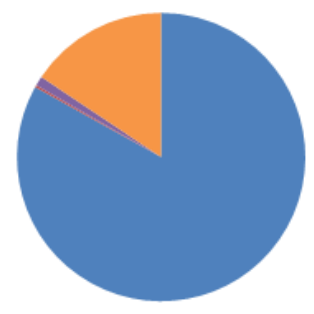

CF1 male
Wheel jammed ( $1^{\text {st }}$ day)
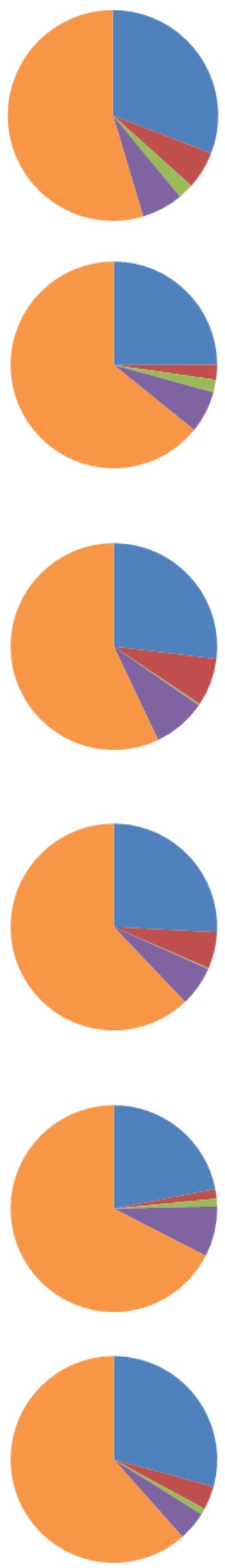

Wheel jammed ( $2^{\text {nd }}$ day $)$
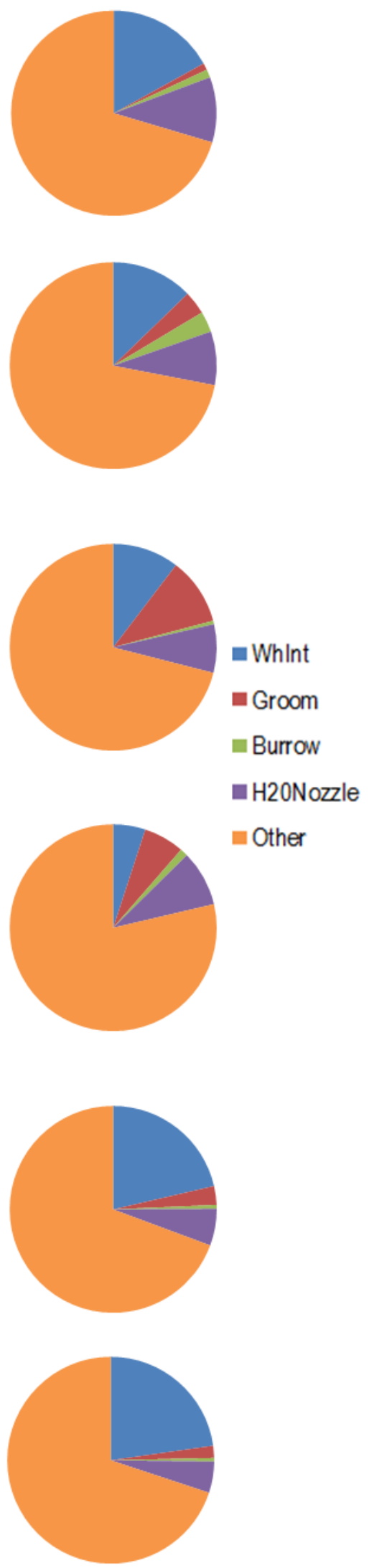
Fig. 4. Duration (mean \%) of all behaviors by sex and day. Note: WhInt, running or sitting on wheel; H2ONozzle, interact with water nozzle; Other, includes cage exploration and climbing on top of jammed wheel. 
A

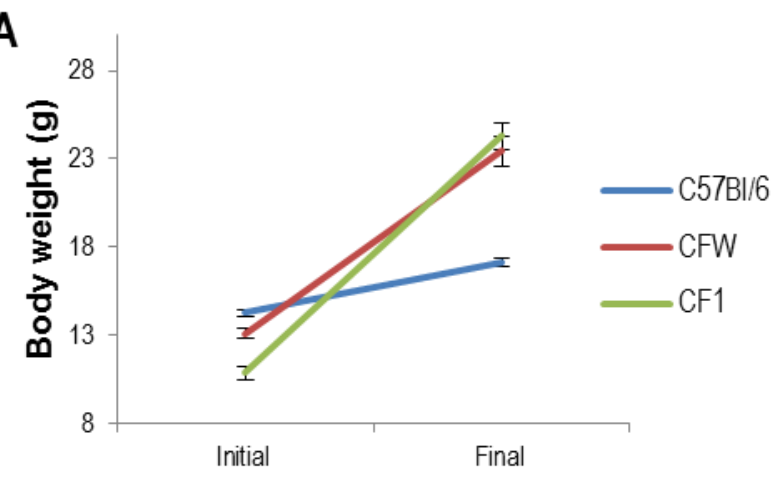

B

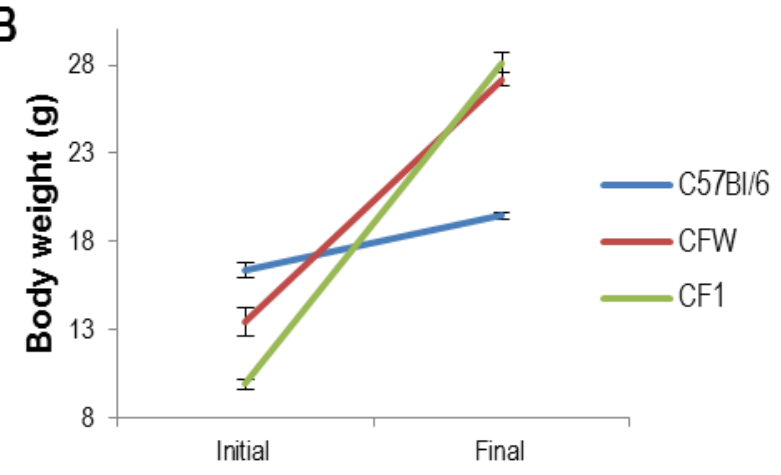

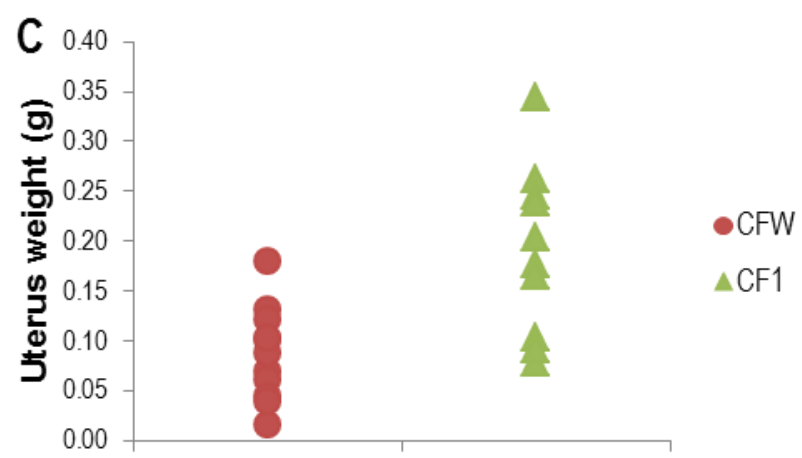

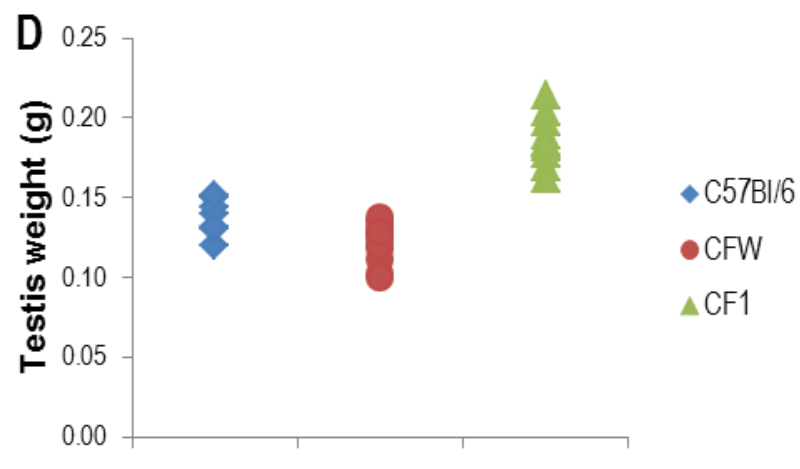

Fig. 5. Mouse morphology. A) Female initial and final body weight (g) by strain (mean \pm SEM), B) Male initial and final body weight (g) by strain (mean \pm SEM), C) Scatter plot of uterine weights (g), D) Scatter plot of paired testes weights (g). Note: Uterine weights were not collected for C57BL/6. 


\section{CHAPTER 3}

\section{EVALUATING SEX DIFFERENCES AND THE EFFECT OF PERINATAL TESTOSTERONE IN A MOUSE MODEL OF AUTISM}

\section{Introduction}

Autism Spectrum Disorders (ASD) are heterogeneous, behaviorally defined conditions characterized by impairments in social interaction, communication, and limited range of interests and activities (Muhle et al., 2004). The prevalence of this disease has been on the rise over the last decade (Center for Disease Control and Prevention, 2016). In many cases the cause of ASD is unknown, with numerous causal mechanisms under investigation, but most researchers agree that autism is a multifactorial condition (Casanova, 2007; Rutter, 2005). ASD is heavily biased towards males (Auyeung et al., 2010), with ratios ranging from 4:1 for classic autism to 11:1 in individuals with Asperger Syndrome (Baron-Cohen et al., 2011). Research into the reasons for this disparity has been limited, but tends to focus on factors that could increase vulnerability in males or, conversely, are protective in females (Alycia K. Halladay, 2015; Baron-Cohen et al., 2011). A common theme in autism research is the 3hit or triple hit hypothesis (Casanova, 2007; Davis \& Pfaff, 2014). As ASD is proposed to result from multiple, variable factors, the sex bias in autism is likely the result of more than one factor that either increases the vulnerability of males or protects females. Using a modified triple hit design, we aimed to determine if prenatal exposure to an ASDinducing teratogen during a vulnerable developmental window, maleness, and additional 
testosterone produced a more severe ASD-like phenotype in mice than those with fewer hits.

Baron-Cohen and colleagues developed the Extreme Male Brain (EMB) Theory, suggesting that ASD is the result of an over-expression of psychological/physiological properties of the male brain, implying males are more likely to display the ASD phenotype (Baron-Cohen et al., 2011). Recent evidence suggests the EMB phenotype could result from elevated levels of steroidogenic activity, including fetal testosterone (fT) (Baron-Cohen et al., 2014), rather than an androgen receptor abnormality or sexually dimorphic gene expression unrelated to fT (Baron-Cohen et al., 2005). Other conditions resulting in abnormal prenatal hormone levels suggest elevated androgens during fetal development could play a role in ASD etiology. Congenital adrenal hyperplasia $(\mathrm{CAH})$, which causes elevated prenatal adrenal androgen production, results in higher scores on the autism-spectrum quotient $(\mathrm{AQ})$ and lower vocabulary scores in females with the condition compared to unaffected female siblings (Knickmeyer et al., 2006). Mothers with polycystic ovary syndrome (PCOS) resulting in abnormally high blood testosterone during pregnancy, produced children with higher total AQ scores compared to controls, with some measures only reaching significance in daughters (Palomba et al., 2012). Multiple studies have correlated fT levels from amniotic fluid samples with ASD indicators, such as autistic traits, restricted interests, poorer quality social relationships, and reduced empathy (Auyeung et al., 2010; Chapman et al., 2006; Knickmeyer et al., 2005). Despite formulation of several testable hypotheses, the underlying cause for the sex bias remains undetermined (Auyeung et al., 2012). 
Fetal exposure to valproic acid (VPA, 2-propyl-pentanoic acid), a drug used in the treatment of epilepsy and bipolar disorder (Roullet et al., 2013), was found to increase risk of major congenital malformations and reduce cognitive abilities (Meador \& Loring, 2013), as well as increase risk of ASD (Christensen et al., 2013). VPA acts to increase gamma-aminobutyric acid synthesis and availability (Dufour-Rainfray et al., 2010; Loscher, 1999) and as a histone deacetylase inhibitor, providing a mechanism to influence gene expression (Dufour-Rainfray et al., 2010). The association between VPA and ASD prompted researchers to develop exposure to VPA as an animal model of ASD (Roullet et al., 2013). Multiple studies in rodents have found that prenatal VPA exposure leads to ASD-like behaviors, including increased repetitive behaviors, delayed physical development, reduced olfactory discrimination, poorer social interaction, increased anxiety, and deficits in ultrasonic vocalization (Kataoka et al., 2013; Roullet et al., 2013; Roullet et al., 2010). In addition, VPA treatment in rodents is associated with decreased cerebellar volume and Purkinje cell number (Ingram et al., 2000), reductions in neuroligin 3 mRNA (Kolozsi et al., 2009) and brain-derived neurotrophic factor (BDNF) mRNA expression (Roullet et al., 2010), and increased corticosterone levels and diminished immunological activity (Schneider et al., 2008). Sex differences in postnatal behavior and neural development have been reported in VPA treated rodents, wherein males display reduced nociception reactivity, heightened anxiety, social interaction deficits (Kataoka et al., 2013; Schneider et al., 2008), delayed motor development (Wagner et al., 2006), and cell loss in the somatosensory cortex (Hara et al., 2012). With numerous studies providing evidence of correlations among elevated fT, brain development, and the ASD phenotype, further investigation into this link was 
warranted (Gore et al., 2014). A better understanding of the relationship among testosterone, ASD risk, and sex can be gained from a direct manipulation of perinatal testosterone (pT). Because prenatal VPA causes sex-specific differences in both behavior and morphology, it was utilized as the autism-inducing insult in this study. Using a modified triple hit design, we sought to determine if prenatal exposure to VPA during a vulnerable developmental window, maleness, and increased testosterone produced a more severe ASD-like phenotype in mice than those with fewer hits. This will allow us to better understand sex differences in VPA effects on brain morphology and behavior and to determine if $\mathrm{pT}$ 's masculinization/de-feminization properties increase female vulnerability to the ASD-inducing insult.

\section{Materials and Methods}

\subsection{Animals}

Animal care was in accordance with the Guide for the Care and Use of Laboratory Animals: Eighth Edition (NRC 2011) and procedures were approved by our Institutional Animal Care and Use Committee. Swiss Webster (CFW) mice were purchased from Charles River Laboratories across 3 separate cohorts in late 2015 and early 2016. This strain was selected because it is commonly used as a general multipurpose model and was used in our previous work investigating sex differences in an autism-relevant wheel running assay (Gordon \& Corbitt, 2015). The mice were received from the vendor at approximately 8-10 weeks of age and housed separately by sex during a week-long acclimation period. All animals were maintained in our animal facility on a 12:12 light/dark cycle and were provided with TekLad 2016 rodent chow and tap water ad lib. Following the acclimation period, we paired two dams with each sire and housed them 
together for breeding. The first day of gestation (E0) was determined based on the presence of a mating plug, at which point dams were separated from sires and housed singly for the remainder of gestation.

We used a between-litter treatment (VPA) and a within-litter treatment (testosterone propionate, TP), see Table 1. Pregnant dams were injected with either 500 mg/kg VPA in saline (i.p.) or vehicle alone (Veh1) on day E12.5 (Hara et al., 2012; Kataoka et al., 2013). Fourteen pregnant dams were separated across 3 cohorts; cohort 1 had only 3 pregnant dams due to VPA litter resorption, cohort 2 had 6 dams, and cohort 3 had 5 dams. Day of birth was noted as postnatal day 0 (P0), at which point we counted pups and checked for stillbirths. On postnatal day 1 (P1), ano-genital (AG) distance was measured and a $2 \mathrm{~mm}$ tail clip was taken after analgesia for Sry detection via PCR, protocol modified from Meeker et al. (2007), in order to identify the sex of each pup. In addition, the animals were weighed and marked with permanent marker as a temporary identifier. On P2, each pup was given a unique tattoo mark using Ketchum animal tattoo paste and a 25 gauge needle for identification throughout the remainder of the project. Following an unusually large litter of 15 pups in cohort1 that had to be excluded, all litters were culled to 10 pups on P2 for cohorts 2 and 3. Approximately half of the offspring (divided equally by sex) from each litter received $100 \mu \mathrm{g}$ TP in $25 \mu$ l peanut oil (s.c.), while the others received peanut oil vehicle only (Veh2). Note that TP was administered on postnatal day 2 because the critical period when male rodents produce testosterone occurs immediately after birth, rather than prenatally as in humans (Hines, 2006). The within-litter and between-litter treatments can also be thought of in accordance with previous "3-hit" or "multiple-hit" hypotheses of autism etiology 
(Casanova, 2007; Pfaff, Rapin, \& Goldman, 2011; Schaafsma \& Pfaff, 2014), wherein in our study the hits are 1)VPA treatment, 2) TP treatment, and 3) "maleness." Hence, a VPA-TP treated male would be considered to have 3 hits, while a Veh1-Veh2 treated female would have 0 hits. All offspring underwent behavioral testing (see section 2.2) and were used for brain measures (see section 2.3). Some males and females from the Veh1 litters that also received Veh2 were used as novel mice in sociability testing; since these novel mice underwent multiple rounds of social exposure, they were not used to measure sociability.

Following the behavioral testing (P31), 80 mice from all cohorts underwent intracardial paraformaldehyde perfusion following standard protocols for morphological endpoints. A small sample of 16 mice from cohort 3 was decapitated under full anesthesia and their brains collected for a separate study.

\subsection{Behavioral Testing}

We selected our behavioral tasks to be relevant to cerebellum $(\mathrm{Cb})$ impairment and the core behavioral deficits associated with ASD. The motor development tests assess delays resulting from VPA's effect on $\mathrm{Cb}$ morphology; other studies have shown deficits in motor development following prenatal VPA treatment in mice (Wagner et al., 2006). The open field and elevated plus maze measure general locomotion, which may be influenced by $\mathrm{Cb}$ development, along with anxiety-like behavior, which has shown to be increased following VPA treatment (Kataoka et al., 2013; Mehta, Gandal, \& Siegel, 2011; Schneider et al., 2008) and is common in ASD (White, Oswald, Ollendick, \& Scahill, 2009). Karvat and Kimchi (2012) used the instinctive wheel running propensity in mice to develop a wheel running assay (WRA) relevant to autism-like behaviors 
(Karvat \& Kimchi, 2012). It assesses key features of ASD including rigidity to change habits, repetitive stereotypical behaviors, and impaired social interactions. In our previous work we showed no baseline sex differences on measures of the WRA in prepubertal mice (Gordon \& Corbitt, 2015), but differential vulnerability to VPA could induce sex differences. Using the VPA rodent model, others have shown increased repetitive behaviors and social interaction deficits (Gandal et al., 2010; Mehta et al., 2011; Schneider et al., 2008), which are assessed using the WRA.

Motor development tests. The mice underwent a series of tests to assess motor development, including surface righting, mid-air righting, negative geotaxis, and hanging grip strength (Wagner et al., 2006). For the surface righting test on P3-8, pups were placed on their backs and the time taken to turn over to all four paws was recorded. If animal did not right itself in $30 \mathrm{~s}$, this time was recorded. For the mid-air righting on days P13-19, pups were held with ventral side up then released $30 \mathrm{~cm}$ above a foam pad. Ability to right was scored if the pup landed on all four paws. Three attempts were given each day to produce possible scores ranging $0-3$ each day. The hanging grip strength task was also performed on P13-19; mice were suspended by all paws grasping a wire mesh placed horizontally $30 \mathrm{~cm}$ above a foam pad. The time spent hanging before dropping to the pad was recorded for the grip strength test with a maximum of $60 \mathrm{~s}$ for each trial. Tests were modified from Wagner et al. (2006). Mice were weaned on P21 and separated by sex, at which point they were housed with littermates, up to 5 per cage. They were given one day to acclimate to new housing before continuing with behavioral trials on P23. 
Open field test/Elevated-plus maze. These tests were used to measure general locomotion, activity, and anxiety-like behavior (Gandal et al., 2010; Kataoka et al., 2013; Schneider et al., 2008). For the open field test (P23), mice were placed in a well-lit novel black plexiglass cage $(37 \mathrm{~cm} \times 37 \mathrm{~cm} \times 37 \mathrm{~cm})$ with overhead camera for $5 \mathrm{~min}$. The parameters measured using EthoVision ${ }^{\circledR} 3$ Pro software (Noldus Information Technology) included: distance traveled, velocity, and time spent in central vs edge zones. The cage was cleaned with ethanol between mice to remove odor cues. The elevated plus maze has four arms $(10 \mathrm{~cm}$ x $50 \mathrm{~cm})$ placed at $90^{\circ}$ angles and mounted $\sim 50$ $\mathrm{cm}$ off the ground. Two of the opposite arms are enclosed by walls, while the other 2 arms remain open to light. Each mouse was placed in the center of the maze and then recorded with an overhead camera for 5 min. The amount of time spent in closed vs. open arms and the number of times entering open arms were the primary measures, but distance traveled and velocity were also measured using EthoVision ${ }^{\circledR} 3$ Pro software as general locomotor indicators. The maze was cleaned with ethanol between mice to remove odor cues.

Wheel running assays. The wheel running assays, modified from Karvat \& Kimchi (2012), assess routine gaining and maintenance, cognitive rigidity, repetitive behaviors, and social interaction with a novel age and sex-matched mouse (Karvat \& Kimchi, 2012). These assays were performed over 6 days (P25-P30) and used a plexiglass cage ( $34.5 \mathrm{~cm} \times 23 \mathrm{~cm} \times 19 \mathrm{~cm}$, covered) lined with corncob bedding fitted with a standard plastic mouse running wheel $(14 \mathrm{~cm}$ diameter) that either freely turned or was jammed by a metal pin; the layer of bedding was changed and the entire cage cleaned with ethanol between mice. An observer blind to treatment group assessed the following 
using Noldus Observer software version 4.1. Stage 1: gaining and maintaining a routine. Each mouse spent 15 minutes in the cage; latency to begin and duration of wheel running were measured each day for 3 days. Stage 2: repetitive behavior. Time spent digging in the bedding/grooming was compared for days the wheel was free vs. jammed. Stage 3: cognitive rigidity. Time spent interacting with the wheel when it was free (days 1-3) and when it was jammed (days 4-5) was measured. Stage 4: social deficit. Lastly, we tested for social deficits by comparing the time spent by the study mouse with the jammed wheel versus engaging in social investigation with a novel mouse (same cohort and sex as focal mouse, but from different litter) on day 6 . The novel mice were place into the arena first; the study mice were then introduced. Only mice from Veh1 litters that also received Veh2 were used as social mice; because they had been exposed to the social test for other litters, these mice were not included in social trials for their own litters.

\subsection{Brain Measures}

Measures of brain development focused on the $\mathrm{Cb}$ because it is the brain area most often affected in ASD (Wang, Kloth, \& Badura, 2014). Cerebellar volume differences are common in autistic patients, frequently with reduced Purkinge cell numbers (Casanova, 2007; Palmen, van Engeland, Hof, \& Schmitz, 2004). VPA rodent models also show reductions in Purkinje cell number and $\mathrm{Cb}$ volume (Ingram et al., 2000), but there are no published data regarding sex differences in the Cb following VPA treatment. Brain development studies focused on regional volumes of the $\mathrm{Cb}$, as this is affected in both ASD (Casanova, 2007; Palmen et al., 2004) and VPA rodent models (Ingram et al., 2000). Following perfusion, brains were removed and placed in fixative (4\% paraformaldehyde) then transferred through graded sucrose solutions prior to rapid 
freezing using dry ice. The samples were stored separately at $-80^{\circ} \mathrm{C}$ until further processing. Each brain was sectioned sagitally at a thickness of $50 \mu \mathrm{m}$ using a cryostat, mounted onto SuperFrost Plus slides, followed by Nissl staining using thionin. Stereological techniques were used to calculate regional volumes (Gundersen, Jensen, Kieu, \& Nielsen, 1999) using ImageJ software. Cerebellar volume was determined using the Cavalieri principle, in which cross sectional area $\left(\mathrm{mm}^{2}\right)$ was multiplied by distance between sections to determine volume $\left(\mathrm{mm}^{3}\right)$ and all sections were added (Gundersen et al., 1999). The $\mathrm{Cb}$ hemisphere and the vermis were measured separately, as Ingram et al (2000) previously found that prenatal VPA had a larger effect on vermis than on $\mathrm{Cb}$ hemisphere in rats.

\subsection{Statistics}

We analyzed results of the study by number of hits and treatment group. Results of all behavioral tests and brain measures were analyzed with a generalized, randomized, block design (GRBD) using a mixed linear model in SAS (9.3, SAS Institute Inc.) where each cohort was designated a 'block' or whole plot factor and the number of hits and litter represented subplot factors. In an attempt to more clearly identify which treatments were contributing to effects, a mixed linear model analysis was also run using the GRBD with cohort as the whole plot factor and the treatments represented as subplot factors. Between-litter treatment was one factor with two variables: VPA or Veh1, and withinlitter treatment was one factor with four variables: TP-male, TP-female, Veh2-male, Veh2-female. Appropriate corrections were made for multiple comparisons (Tukey). We used preliminary analyses to determine effects of interaction terms; if no significant interaction was present, these terms were excluded from further analysis. For the WRA 
the effect of day on behaviors was determined using a two-way ANOVA for treatment group and day. Note: we excluded one litter (cohort 1) from our analyses due to an abnormally large litter size (15 pups), which resulted in the failure to thrive of 2 pups and low body weight across the litter; this outcome prompted us to cull litters to 10 pups at P2 thereafter.

\section{Results}

\subsection{Motor Development Tests}

Surface righting. In the hits analysis, there was a significant litter effect on days P3 and P5 ( $p=0.03, p=0.04$, respectively); this effect appears to be driven by VPA/Veh1 treatment differences across litters. However, the number of hits was unrelated to surface righting time for all days $(\mathrm{p}>0.29)$. Offspring of dams receiving VPA treatment appeared to display longer latencies to surface right compared to offspring of Veh1 treated dams (Fig 1-A). On P3 the average surface righting time for the VPA treatment group was 16.7 $\mathrm{s}$, while the Veh1 treatment group average was $11.8 \mathrm{~s}$. However, the mixed linear model analysis showed these latency values failed to reach statistical significance on all days measured ( $p>0.16)$. There was also no significant effect of sex or TP treatment $(p>0.09)$ and no interaction effects ( $\mathrm{p}>0.11)$ across all days.

Mid-air righting. The mixed linear model analysis of mid-air righting scores by number of hits uncovered a significant effect of hits $(\mathrm{p}=0.04$, Fig 1-B) on P19 with average number of successful righting attempts decreasing from 2.75 for both 0 and 1 hits, to 2.38 for 2 hits, and to 2.0 for 3 hits. There was also a significant effect of litter on $\mathrm{P} 13(\mathrm{p}=0.02)$ and $\mathrm{P} 19(\mathrm{p}=0.001)$, and an interaction effect between hits and litter 
$(\mathrm{p}=0.04)$ on P19. However, analysis of scores revealed no significant effect of VPA treatment, TP treatment, or $\operatorname{sex}(\mathrm{p}>0.11)$.

Hanging Grip Strength. Hanging grip times analyzed by number of hits showed that the effect of hits approached significance for P17 and P18 (p<0.08, Fig 1-C), but no other days ( $>0.27)$. On P17 the longest average hanging grip time was recorded for Veh1-Veh2 females (0 hits, $33.4 \mathrm{~s}$ ) and the shortest average time for VPA-TP males (3 hits, $16.7 \mathrm{~s})$, with pups designated as 1 and 2 hits being intermediate $(22.6 \mathrm{~s}$ and $19.2 \mathrm{~s}$, respectively). On P18 the average hanging grip time ranged from $27.4 \mathrm{~s}$ for 1 hit to $24.5 \mathrm{~s}$ for 2 hits to $16.5 \mathrm{~s}$ for 3 hits; however, the average time for 0 hits was intermediate (22.6 s). The negative geotaxis results are not presented due to infrequency of task completion across all cohorts. There was also a significant effect of litter on all days ( $<<0.03)$, except P13 and P15. Pups receiving VPA treatment in utero appeared to regress in their hanging grip strength ability, implying VPA/Veh1 treatment differences are driving the litter effects. VPA pups displayed similar hanging grip times to Veh1 pups on early trial days P13, P14, and P16 ( $>>0.18)$; however, VPA pups underperformed Veh1 pups with shorter duration for hanging grip time on days $\mathrm{P} 15, \mathrm{P} 17$, and $\mathrm{P} 19(\mathrm{p}=0.004, \mathrm{p}=0.03$, $\mathrm{p}=0.04$, respectively) (Fig 1-D). This effect was not influenced by TP treatment or sex $(\mathrm{p}>0.33)$.

\subsection{Open Field/Elevated Plus Maze}

Open Field. The hits analysis showed a significant effect of litter and an interaction effect between litter and hits on velocity in the center zone $(\mathrm{p}<0.0001$, $\mathrm{p}=0.0006$, respectively). There was also a significant effect of litter on frequency of entry into the center $(\mathrm{p}=0.0006)$ and edge zones $(\mathrm{p}=0.0024)$, but it does not appear to be driven 
by treatment group, as there was no significant effect of VPA treatment, TP treatment, or sex on frequency, duration, or latency of zone entries in the center or edge zones (all pvalues $>0.12)$; there was also no effect on velocity or total distance moved ( $p>0.28)$. Overall, neither VPA nor TP treatment appeared to affect general locomotion or anxiety (as measured by frequency and duration in center vs. edge zone) in the open field trials.

Elevated Plus Maze. The mixed linear model analysis revealed a significant effect of litter and hits for frequency of entry into open arms ( $\mathrm{p}<.0001, \mathrm{p}=0.02$, respectively) (Fig 2-A); however, there was no interaction effect ( $\mathrm{p}>0.41$ ). VPA-TP males (3 hits) had the highest number of entries (16.0), while Veh1-Veh2 females had the lowest frequency of entry into open arms (11.1). There was also an effect of litter on frequency of closed arm entries $(\mathrm{p}<0.0001)$. The number of hits approached, but failed to reach, significance for open arm duration ( $\mathrm{p}=0.08$, Fig 2-B). Both litter and hits approached significance for duration spent in the closed arms ( $p>0.08$ for both). Animal velocity in the entire arena displayed a litter effect $(\mathrm{p}<0.0001)$, with number of hits approaching significance $(\mathrm{p}=0.07$, Fig 2-C). The analysis found no significant effect of VPA treatment, TP treatment, or sex on frequency or duration of zone entries in the open or closed arms of the maze ( $p>0.42$, Fig 2-D); there was also no effect on velocity measured for the entire maze $(\mathrm{p}>0.53)$.

\subsection{Wheel Running Assays}

Latency of Behavior. The mixed linear model analysis revealed a significant effect of day for latency to initiate wheel running ( $\mathrm{p}<0.001)$ (Fig 3-A). Across all treatment groups, latency to run was significantly less on day 2 compared to day 1 $(\mathrm{p}<0.001)$. The expected increase in latency to run on day 5 when compared to day 4 was 
only significantly different for Veh1-TP males $(\mathrm{p}=0.006)$, but was not different for all other treatments $(\mathrm{p}>0.08)$. Our analysis showed no significant effect of VPA treatment, TP treatment, or sex on latency of behavior for any behaviors measured (i.e., wheel running, wheel interaction, grooming, burrowing, $p>0.20)$. On day 1 there was no difference between any treatment group, sex, litter, or number of hits for latency to run on the wheel $(p>0.20)$ or in latency of stereotypical behaviors, including burrowing $(p>0.31)$ and grooming $(\mathrm{p}>0.70)$. Social interaction initiation was assessed by the latency of the mouse to contact or begin following a sex-matched novel mouse (Veh1-Veh2). Again, we found no difference between any treatment groups or number of hits in latency to contact $(\mathrm{p}>0.11)$ or follow $(\mathrm{p}>0.18)$ the novel mouse (Fig 3-B,C).

Duration of Behavior. Our analysis of the duration of behaviors used the percentage of time the mouse spent performing each behavior. The results of our mixed linear model analysis showed a significant effect of day on wheel running for all treatment groups $(\mathrm{p}<0.0001$, Fig $4-\mathrm{A})$. In a comparison of both wheel running (time spent actively running on the wheel) and wheel interaction (time spent in the wheel, whether running or stationary) duration times for the last day the wheel was free (day 3) vs. the first day the wheel was jammed (day 4), and the first day the wheel was jammed vs. the second day the wheel was jammed (day5), all p-values reached significance $(\mathrm{p}<0.0001)$.

We also compared wheel running and wheel interaction duration (\%) for our treatment groups across all days for days the wheel was free vs. jammed. In general, we found no significant effect of VPA treatment, TP treatment, sex, litter, or hits on duration of wheel running $(\mathrm{p}>0.34)$ or wheel interaction $(\mathrm{p}>0.63)$ across all days. When we looked at these behaviors by day, we found a significant effect of hits on duration of wheel 
interaction for day 5 , the second day the wheel was jammed $(\mathrm{p}=0.04)$. The average percentage of time spent interacting with the wheel ranged from 14.2\% for Veh1-Veh2 females ( 0 hits) to $22.0 \%$ for VPA-TP males ( 3 hits), with mice designated as receiving 1 and 2 hits being intermediate $(20.1 \%, 17.5 \%$ respectively). However, there was no apparent effect of VPA treatment, TP treatment, sex, or litter on wheel running $(\mathrm{p}>0.10)$ or wheel interaction ( $>0.22)$ for any other comparison by day.

Our analysis revealed that duration (\%) of stereotypical behaviors, including burrowing $(\mathrm{p}=0.035)$ and grooming $(\mathrm{p}<0.0001)$, was higher when the wheel was jammed (day 4) when compared to a day the wheel would freely turn (day 3), see Fig 4-B for grooming. Our duration analysis of stereotypical behaviors displayed an effect of litter $(\mathrm{p}=0.03)$ and hits $(\mathrm{p}=0.02)$ on duration of grooming on day 1 . VPA-TP males ( 3 hits) spent the most time grooming (4.4\%), while those designated as 1 hit groomed the least (1.5\%). We also found an effect of litter on grooming for day $5(\mathrm{p}=0.003)$ with the interaction of litter by hits approaching significance $(\mathrm{p}=0.06)$. Litter had a significant effect on burrowing behaviors for day $4(\mathrm{p}=0.009)$ and there was a litter by hits interaction effect on day 1 burrowing behaviors $(\mathrm{p}=0.03)$.

As part of our social interaction test we measured duration of contact and following behaviors initiated by the subject mouse. Although the effect of VPA treatment appeared to cause a nominal increase in the duration of contact, this effect failed to reach significance ( $\mathrm{p}=0.19$, Fig 4-C). There was also no effect of sex by TP or hits ( $\mathrm{p}>.56)$ on duration of contact with the novel mouse; however, there was a significant effect of litter $(\mathrm{p}<0.001)$. Following behavior was also affected by litter $(\mathrm{p}<0.0001)$, but not hits ( $>0.43$, Fig 4-D). Duration of following did not vary by VPA treatment $(\mathrm{p}=0.18$, Fig 4- 
E), but there was a significant effect of $\operatorname{sex}(\mathrm{p}=0.03)$ with females, regardless of TP treatment, spending more time following the novel mouse (1.7\%) than males did $(0.6 \%)$. Lastly, we evaluated the duration of social interaction (contact + following), with only litter $(\mathrm{p}<0.0001)$ reaching significance.

\subsection{Brain Measures}

We calculated volume for the entire $\mathrm{Cb}$, the cerebellar vermis, and cerebellar hemispheres, along with the volume of the granular layer of the cerebellar vermis and hemispheres. There was no effect of number of hits on volume measures $(\mathrm{p}>0.65)$. However, there was a significant litter effect on all measures: $\mathrm{Cb}$ volume $(\mathrm{p}<0.0001)$, vermis volume $(\mathrm{p}=0.0005)$, hemisphere volume $(0.0004)$, vermis granular layer $(0.0005)$, and hemisphere granular layer (0.0010). We did not find any effect of VPA treatment ( $>0.81)$, sex by TP treatment ( $p>0.52)$, or an interaction effect between these treatments ( $>0.45$ ) on measures of volume from any region of interest.

\section{Discussion}

Valproic acid is commonly used to treat epilepsy and bipolar disorder, but is associated with congenital birth defects and impaired cognition with fetal exposure (Meador \& Loring, 2013; Roullet et al., 2013). Previous studies have assessed the effect of prenatal VPA exposure on a battery of behaviors (Kataoka et al., 2013; Roullet et al., 2013; Roullet et al., 2010; Schneider et al., 2008), brain morphology (Hara et al., 2012; Ingram et al., 2000), and gene expression (Kolozsi et al., 2009; Roullet et al., 2010) in rodents with some studies finding sex differences in postnatal behavior (Kataoka et al., 2013; Schneider et al., 2008; Wagner et al., 2006) and sex-dependent changes in brain morphology (Hara et al., 2012). To our knowledge, there have been no previous reports 
studying the effects of VPA in combination with exogenous $\mathrm{pT}$ aimed at understanding how pT influences vulnerability to an ASD-inducing insult. We anticipated that the VPAtreated mice, as a result of altered neural development, would exhibit behaviors similar to those seen in ASD, such as motor impairment or delay, repetitive behaviors, decreased social interaction, heightened anxiety, and increased cognitive rigidity (inability to forgo a routine). Secondly, due to higher levels of endogenous fT, we proposed that males treated with VPA would have more significant behavioral abnormalities than VPAtreated females. Finally, our study was designed to assess the effect of exogenous pT, and whether masculinizing females would increase the effect of VPA, resulting in behaviors similar to those of VPA-treated males. We also hypothesized that males receiving both TP and VPA (3 hits) may display increased abnormalities in behavior due to the enhanced masculinization effect of exogenous testosterone.

Cerebellar injury is the largest single, non-heritable risk for ASD with a risk ratio calculated at 36x the general population (Wang et al., 2014). Early disruption of cerebellar growth is positively correlated with ASD (Courchesne et al., 2001; Hashimoto et al., 1995) and subsequent impairment of regional volumetric growth in the contralateral cerebrum (Limperopoulos et al., 2014). Furthermore, the $\mathrm{Cb}$ is one of the brain regions most often disrupted in those with $\mathrm{ASD}$, at the microscopic and gross levels (Casanova, 2007; Palmen et al., 2004; Wang et al., 2014). The Cb is well known for its role in balance, posture, and motor function; however, evidence now suggests the $\mathrm{Cb}$ also supports higher function (Wang et al., 2014). Human functional imaging measurements have shown that almost every part of neocortex has an associated region in the $\mathrm{Cb}$ as displayed by measurements of covariation between neocortex and cerebellum, thus $\mathrm{Cb}$ 
abnormality potentially affects other regions implicated in ASD (Wang et al., 2014). A meta-analysis of motor dysfunction also found a large, positive effect that indicates significant motor coordination deficits in ASD participants occurring in approximately $80 \%$ of ASD cases (Fournier, Hass, Naik, Lodha, \& Cauraugh, 2010). In addition, regional differences in $\mathrm{Cb}$ structure are present in five ASD rodent models (Wang et al., 2014), including VPA treatment (Ingram et al., 2000). Accordingly, we selected our behavioral tasks to be relevant to $\mathrm{Cb}$ impairment, along with the core behavioral deficits associated with ASD, and focused on brain morphology measures of the $\mathrm{Cb}$.

We demonstrate that VPA exposure in utero negatively affects motor development, with some measures being most affected in the triple hit group (VPA-TPmale). However, the combined effect of VPA-TP treatment in males appears to reduce anxiety in the EPM, while also inducing a nominal increase in velocity or activity in the maze. In the WRA, females, regardless of treatment group, displayed higher sociability as measured by time spent following a novel mouse. The impact of prenatal VPA on motor development suggests that this treatment has some bearing on cerebellar development; however, this effect did not result in significant volumetric differences in the $\mathrm{Cb}$. The results of the hits analyses suggest that $\mathrm{pT}$ could act through some mechanism to increase vulnerability of males to our autism-inducing insult, but further study is needed to better understand this mechanism.

Dufour-Rainfray et al. (2010) assessed motor development in rats exposed to 600 mg/kg VPA on E9 (Dufour-Rainfray et al., 2010). They found no significant difference in behavior between the VPA and control group across all tests, including righting reflex, gait reflex, negative geotaxis, response to tactile stimulation, and rope suspension 
(Dufour-Rainfray et al., 2010). On the other hand, Wagner et al. (2006) evaluated motor development in BALB/c mice following a $600 \mathrm{mg} / \mathrm{kg}$ dose of VPA on E13 (Wagner et al., 2006). Their findings revealed VPA treatment impaired surface righting and mid-air righting abilities independent of sex and that hanging grip time was reduced in VPA exposed mice, with VPA males outperforming VPA females; however, there was no difference between treatment groups in negative geotaxis or balance beam tasks (Wagner et al., 2006). While our results indicated that surface righting and mid-air righting were unaffected by VPA treatment, sex, and TP treatment, we did find a significant effect of number of hits on the last day of the mid-air righting test with the average number of successful attempts decreasing as number of hits increased. This could indicate that the combined effect of VPA-TP treatment in males caused a regression in mid-air righting ability on P19. In our hanging grip test, both VPA and Veh1 animals displayed similar hang times on the first day of the test. VPA treatment, independent of sex and TP treatment, appeared to reduce hanging grip time for subsequent days of the test with this difference reaching significance on days P15, P17, and P19, consistent with the findings by Wagner et al. (2006) that VPA treatment results in a delay to maturation in this skill (Wagner et al., 2006). The results of the hanging grip task also suggest that increasing number of hits resulted in worse performance on later days of this task; these values approached significance on P18 and P19.

Mehta et al. (2011) treated pregnant C57BL/6Hsd (B6) mice with $600 \mathrm{mg} / \mathrm{kg}$ VPA on E13 and found that mice exposed to VPA exhibited a reduction in the frequency of center zone entries during open field testing from P56-P64 when compared to saline exposed mice; however, duration in the center zone and overall locomotor activity were 
not difference between the two groups (Mehta et al., 2011). Another study using the same mouse strain and VPA treatment regimen found no differences in open field behavior or locomotor activity between treatment groups (Gandal et al., 2010). Our results suggest that VPA treatment, sex, TP treatment, and number of hits did not affect frequency of zone entry or zone duration in the open field trial, indicating no difference between groups in general locomotion or anxiety during this trial.

Schneider et al. (2008) evaluated sexual dimorphic effects of VPA on a battery of tests in Wistar rats treated in utero with $600 \mathrm{mg} / \mathrm{kg}$ VPA on E12.5 (Schneider et al., 2008). For EPM they found similar levels of locomotor activity and frequency of entries into the closed arms when comparing the VPA treatment group to controls, while females were more active than males (Schneider et al., 2008). The male VPA treated rats spent less time and had fewer entries into the open arms of the maze, indicating higher anxiety; VPA females' behavior was similar to that of control males, while control females displayed the lowest measures of anxiety on this task (Schneider et al., 2008). While we found no significant effect of VPA treatment, TP treatment, or sex on frequency or duration of arm entries or an effect on overall velocity, we did find some effects of the number of hits. Opposing the expected pattern of behavior, the Veh1-Veh2 females (0 hits) had the fewest entries into the open arms, and the VPA-TP males (3 hits) had the most entries into the open arms, indicating VPA-TP males (3 hits) displayed the lowest levels of anxiety in this task. The VPA-TP males also had the longest duration in the open arms, also indicating low anxiety; this measure approached significance. Animal velocity also nearly reached significance, with the VPA-TP males displaying the highest average velocity. Hyperlocomotor activity could be one factor leading to an increased 
frequency of entries into the open arms for the VPA-TP males; the study by DufourRainfray (2010) also found hyperlocomotor activity in male rats treated with VPA (Dufour-Rainfray et al., 2010). Increased activity, however, fails to justify the VPA-TP males' longer duration in the open arms of the maze.

Wheel running behaviors have been explored in rodents in many contexts, e.g., locomotor activity, energy balance, obesity, and circadian rhythm (Novak, 2012), and are consistently appraised as rewarding activities (Werme et al., 2002). Karvat and Kimchi (2012) used the wheel running proclivity in mice to develop a behavioral test applicable to autism-like behaviors (Karvat \& Kimchi, 2012). They designed this assay to assess features of autism including rigidity to change habits, repetitive stereotypical behaviors and impaired social interactions; their results showed that BTBR mice, a strain known to exhibit a robust autism phenotype, displayed higher levels of burrowing and grooming, heightened cognitive rigidity, and reduced social interaction (Karvat \& Kimchi, 2012). Our prior work exploring sex differences in pre-pubertal mice found that 3 commonly used strains displayed no impairments in behavior as measured by this assay, nor were any baseline sex differences in behavior evident (Gordon \& Corbitt, 2015).

As shown in our previous study using the WRA to assess sex differences in prepubertal mice, latency to run on the wheel should decrease following the initial introduction of the mice to the novel wheel (Gordon \& Corbitt, 2015). In this study we found that latency to wheel run was shorter on the $2^{\text {nd }}$ day of the assay compared to the $1^{\text {st }}$, indicating all treatment groups displayed normal routine gaining. The WRA is designed to measure cognitive rigidity, which is assessed by indicators of ability to break a routine. We measured cognitive rigidity by comparing latency to run on the wheel on 
day $4\left(1^{\text {st }}\right.$ day jammed $)$ with day 5 ( $2^{\text {nd }}$ day jammed $)$; while latency appeared to increase for the Veh1 groups, this increase was significant only in the Veh1-TP males. This indicates that all treatment groups in our study, except for Veh1-TP males, demonstrated impaired cognitive rigidity or memory, i.e., the mice failed to break routine of wheel access or did not remember the wheel was jammed. We expected indications of cognitive rigidity impairment in VPA treatment groups, but not in our Veh1-treated mice. The behavior of control mice in this study was different than in our previous work where we saw increased latency of hundreds of seconds (Gordon \& Corbitt, 2015). One possible explanation could be increased stress levels, both prenatal and postnatal. All dams, regardless of treatment group received injections on E12.5. While this procedure was performed quickly, it undoubtedly activated the dam's stress response. In addition, pups were separated from dams for brief periods early in development (P2-P8 and P13-P19) to perform tail clip, tattooing, and motor development testing. This separation can lead to increased stress in both the dam and the pups. Many studies have shown cognitive and behavioral deficits in rodents exposed to pre- and post-natal stress (Emack et al., 2008; Franklin et al., 2010; Markham et al., 2010; Mueller \& Bale, 2007).

All mice in this study followed the expected pattern of wheel running duration, with heightened wheel running duration on days the wheel was free compared to those when the wheel was jammed. The mice in our study, regardless of treatment group, also maintained their ability to forfeit the running habit when the wheel was jammed, as displayed by reduced wheel interaction on days 4 and 5 of the assay. While latency measures indicated some increase in cognitive rigidity, reduced wheel interaction on days 4-5 shows that the mice were capable of changing habits. However, on day 5 of the assay 
there was an effect of hits on duration (\%) of wheel interaction (but not wheel running), with Veh1-Veh2 females (0 hits) spending the least amount of time and VPA-TP males (3 hits) the most time interacting with the wheel (data not shown), demonstrating that although VPA-TP males spent less time interacting with the wheel on day 5 compared to day 4 , they spent relatively more time interacting with the wheel on day 5 compared to the other treatment groups. Therefore, the combined effect of VPA, TP and male sex appeared to increase cognitive rigidity on this day.

Previous work by Gandal et al. (2010) using C57BL/6Hsd (B6) mice treated prenatally with $600 \mathrm{mg} / \mathrm{kg}$ VPA on E13 demonstrated that VPA-treated mice had increased repetitive self-grooming behaviors. The aforementioned study by Mehta et al. (2011) also found that rodents exposed to VPA displayed higher levels of self-grooming compared to controls. Our study revealed an effect of hits on self-grooming duration on day 1 with VPA-TP males (3 hits) spending the most time in this activity and the 1-hit group grooming the least. This increase in repetitive behaviors provides evidence that exogenous TP treatment increases vulnerability in males to the effects of VPA. However, this support is limited in scope, as the increase in self-grooming in VPA-TP males is limited to day 1 of the WRA. Our analysis of VPA treatment, TP treatment, and sex failed to provide more information regarding which treatment resulted in increased grooming, as no factor reached statistical significance for latency to initiate stereotypical behavior, i.e., self-grooming or burrowing. We also saw that overall, self-grooming and burrowing behaviors were diminished when the wheel was free when compared to days the wheel was jammed, indicating all treatment groups preferred wheel running to performing these stereotypical behaviors. 
Given that ASD is characterized by impairments in social interaction, numerous studies have measured social behaviors in VPA rodent models. The studies by Gandal et al. (2010) and Roulett (2010) found mice treated with VPA displayed a decreased social preference in comparison to the control mice (Gandal et al., 2010; Roullet et al., 2010). Kim et al. (2011) demonstrated that Sprague-Dawley rats exposed at E12 to $400 \mathrm{mg} / \mathrm{kg}$ VPA had lower levels of sociability and social preference (K. C. Kim et al., 2011). The work by Dufour-Rainfray (2010) indicated VPA rats initiated fewer social approaches than controls, but had no other signs of social deficits (Dufour-Rainfray et al., 2010). In Kataoka's (2013) study, researchers found a sexually dimorphic response to VPA treatment, with only male mice exhibiting reduced social behavior (shorter duration of sniffing); however, there was no effect on allogrooming or aggression in either sex (Kataoka et al., 2013). Conversely, Narita et al. (2010) found no differences in social behaviors (sniffing, dominant status, allogrooming, pinning, chasing, or aggressive behaviors) between rats exposed to VPA $(800 \mathrm{mg} / \mathrm{kg})$ on E9 and control rats (Narita et al., 2010).

Our previous work using this WRA to assess wheel running and social behaviors in 3 strains of mice revealed no sex difference in social behaviors (Gordon \& Corbitt, 2015). In the current study we found no difference in latency to initiate social behaviors, e.g., contact or following. However, females in this study spent more time following the novel mouse than males; this effect was consistent across all treatment groups. The sex difference in following behaviors could indicate that males in our study were more influenced by the pre- and post-natal stress that was inherent to the design of our study. Other studies have also reported a sexually dimorphic response to maternal stress (Emack 
et al., 2008; Franklin et al., 2010; Markham et al., 2010; Mueller \& Bale, 2007). Previous studies suggest that VPA treatment has a negative effect on social behavior (DufourRainfray et al., 2010; Gandal et al., 2010; K. C. Kim et al., 2011; Roullet et al., 2010), especially in males (Kataoka et al., 2013). Therefore, the VPA treated mice in our study, males in particular, should be expected to spend less time in social contact. Our results revealed that Veh1-Veh2 females (0 hits) spent the shortest duration (\%) and the VPA-TP males (3 hits) had the longest duration (\%) of contact with the novel mouse. Conversely, VPA-TP males spent the least amount of time participating in following behaviors, while the 1-hit group spent the most time pursuing the novel mouse. The effect of hits on contact and following behaviors, however, failed to reach significance. Since our analysis used percentage of time spent performing behaviors, we analyzed the combined duration of all social behaviors (contact + following), but found social behavior was only influenced by litter. The overall trend of social behaviors was opposite our expectations with the Veh1-Veh2 females spending the least amount of time interacting socially, while the VPA-TP males spent the most time. One possible explanation is that we used a novel method to assess social interaction in the VPA rodent model by choosing the WRA. Many of the prior studies have used social preference tasks.

Our study produced varied results across the battery of behavioral tasks. Assessment of motor development tests provided support for our hypotheses. We found both delayed development and regression of skill in motor development tasks in VPAtreated pups, which supports previous work in this field. We also found some indication that the combined effect of VPA, TP, and maleness (triple hit) resulted in worse motor development outcomes compared to other treatment combinations with fewer hits. 
Demonstrating a lack of support for our hypotheses, we found no anxiety impairments in the open field task, and the EPM results revealed that VPA-TP males showed decreased, rather than the expected increased, anxiety. The WRA assay results were mixed. We found no major effects on cognitive rigidity in our WRA assay, aside from VPA-TP males spending more time with the wheel compared to other groups on day 5. Increased stereotypical behaviors were limited to day 1 of the WRA when VPA-TP males displayed increased self-grooming behaviors. Surprisingly, the social interaction component of the WRA trended towards VPA-TP males spending the most time in social interaction (18.8\%), which represents $27.6 \mathrm{~s}$ more social contact than the least social group (Veh1Veh2 females). Finally, we found no reduction of $\mathrm{Cb}$ volume, either regional or total cerebellar, as predicted based on previous findings. On the other hand, we found impairments in motor development, which are thought to be associated with cerebellar maturation. Therefore, we cannot rule out an effect of VPA on $\mathrm{Cb}$ function even though we did not find significant differences in our gross volume measures.

One weakness of our study is the use of a drug as the autism-inducing insult. With the variability seen across human and animal studies resulting from different VPA doses, the timing of administration, and delivery method, it is important to consider the pharmacokinetics of VPA and how that relates to our murine model. In clinical studies, VPA administration during pregnancy is associated with adverse outcomes, such as congenital malformations, cognitive disability, and risk of ASD with risk of such negative outcomes increasing with higher daily doses of VPA (Roullet et al., 2013). In rodent studies, all VPA doses tested (200-800 $\mathrm{mg} / \mathrm{kg}$ ) alter neurodevelopment; yet, a precise dose threshold for this ASD model has not been clearly identified (Roullet et al., 
2013). While the half-life in humans is $9-18$ hours, the rat displays dose-dependent nonlinear elimination kinetics and a lower half-life of 2-5 hours and the elimination halflife in the mouse is even faster, at 0.8 hours (Loscher, 1999). The brain/plasma ratios of VPA are similar in all species investigated, but the other pharmacokinetic differences lead to the higher VPA doses needed in rodents to obtain equivalent plasma levels as in humans (Loscher, 1999). The rodent VPA model has been studied for many years; however, the timing, dose, and delivery method of VPA is inconsistent across studies, resulting in variability of autism-like behavior and brain development outcomes in these models (Gandal et al., 2010; Hara et al., 2012; Kataoka et al., 2013; Mehta et al., 2011; Roullet et al., 2010; Wagner et al., 2006). Due to the fact that the endogenous testosterone surge in rodents occurs immediately after birth, we chose to give our TP treatment at this time. However, this resulted in the VPA and TP treatments being separated by $8-10$ days. It is unknown how this lag between treatments affects the practicality of this design. Another weakness of this study is the possible confounding stress effect. While we could ensure that all treatment groups experienced the same procedures which were likely to induce stress (prenatal maternal stress from VPA or Veh1 injection and postnatal stress from handling and maternal separation), we cannot confirm that all reactions to stress or separation were the same across litters. Differences in reactions to stress may help explain litter effects seen in our hits analyses, which were not always due to treatment (VPA/Veh1) differences among litters.

While the results of this study indicated effects of VPA treatment and sex on multiple behavioral tasks, we did not find the expected associations between the combinations of VPA and TP treatments with sex. By also analyzing data by hits, we 
found indications that the combined effects of VPA and TP treatment in males increases the severity of behavioral impairment in some tasks. While we show that the triple hit of VPA-TP treatment in males appears to negatively impact the development of some skills (motor development, cognitive rigidity, and stereotypical behaviors) more severely in this group compared to other treatments, some outcomes were unaffected (social interaction) or even enhanced in this group (anxiety). The mechanisms underlying the previously reported sexual dimorphic effects of VPA remain unclear. Further studies in rodents are needed to more clearly identify the appropriate timing, dose, and delivery method of VPA and TP to achieve a more reliable model of ASD and to better understand the mechanism by which increasing $\mathrm{pT}$ intensifies vulnerability to autism-inducing insults, such as VPA, in males. 
Table 1. Treatment (Tx) Groups

\begin{tabular}{cccccc}
\hline Dam Tx (E12.5) & Pup Tx (P2) & Sex & Final Tx Groups & \# of Hits & n \\
\hline VPA & TP & M & VPA + TP & 3 & 10 \\
& TP & F & VPA + TP & 2 & 11 \\
& Veh2 & M & VPA + Veh2 & 2 & 11 \\
& Veh2 & F & VPA + Veh2 & 1 & 11 \\
& TP & M & Veh1 + TP & 2 & 14 \\
Veh1 & TP & F & Veh1 + TP & 1 & 14 \\
& Veh2 & M & Veh1 + Veh2 & 1 & 12 \\
& Veh2 & F & Veh1 + Veh2 & 0 & 13 \\
& & & & & \\
\hline
\end{tabular}



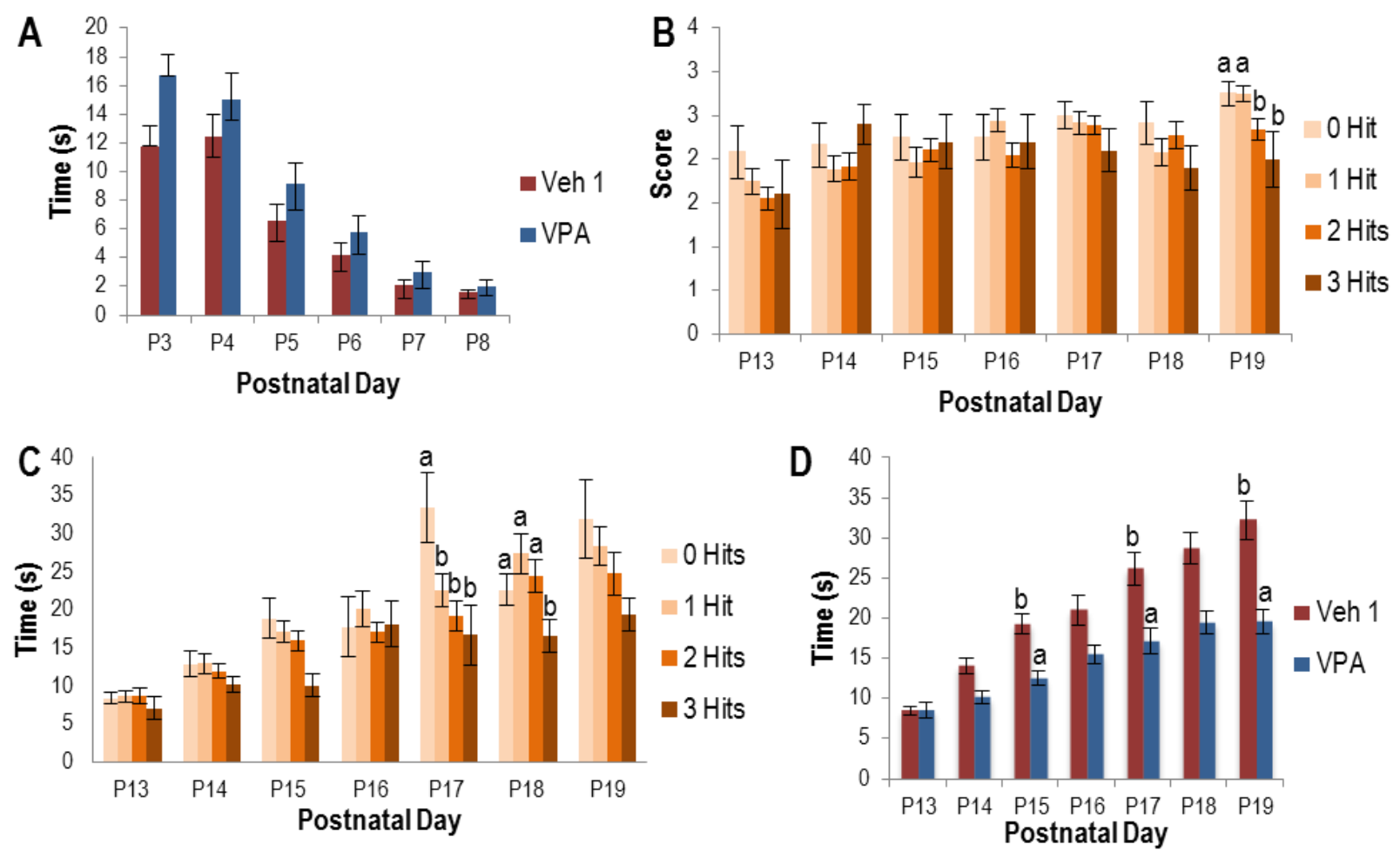

Figure 1: Duration or score of motor development behaviors by day (mean \pm SEM). A) surface righting time by VPA treatment, B) mid-air righting score by number of hits, C) hanging grip time by number of hits, D) hanging grip time VPA treatment. ${ }^{\text {ab }}$ Differing superscripts indicate statistically significant difference $(\mathrm{p}<0.05)$. 

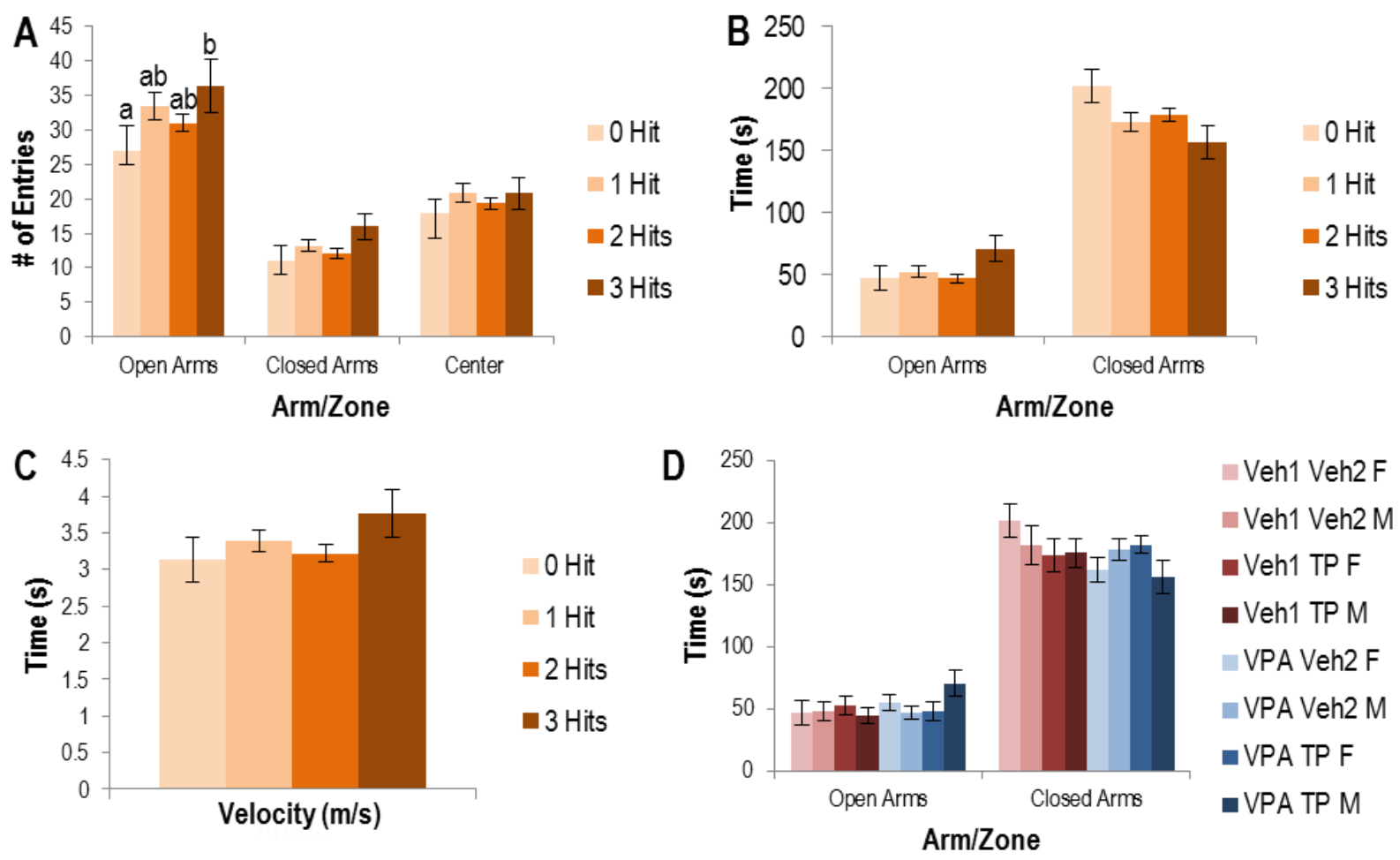

Figure 2: Frequency, duration, and velocity measures from EPM (mean $\pm \mathrm{SEM})$. A) frequency of zone entry by number of hits, B) duration in open vs. closed arms by number hits, C) velocity in arena by number of hits, D) duration in open vs. closed arms by treatment group. ${ }^{a b}$ Differing superscripts indicate statistically significant difference ( $p$ $<0.05)$. 

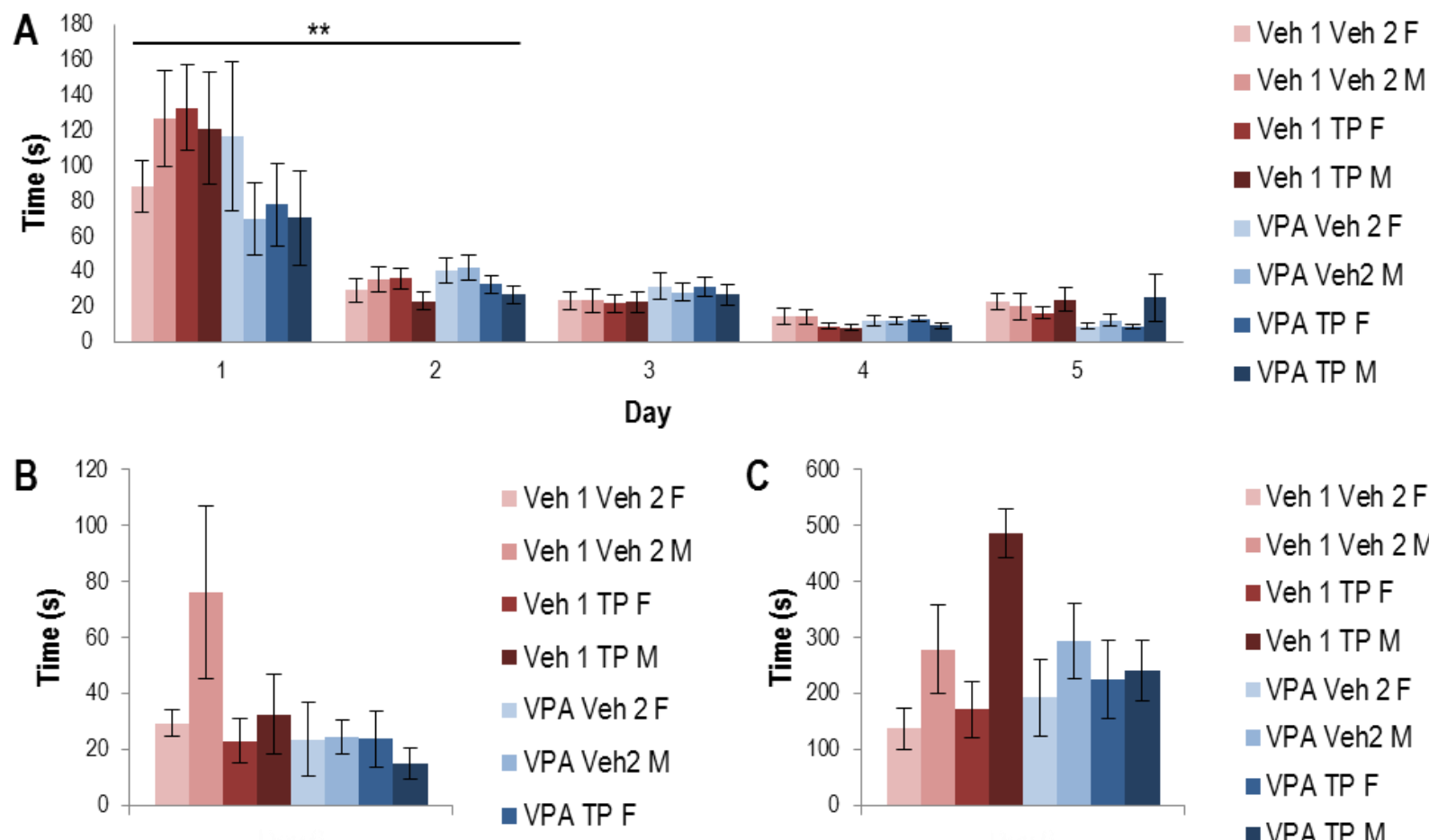

$\square$ Veh 1 Veh $2 \mathrm{~F}$

- Veh 1 Veh $2 \mathrm{M}$

-Veh 1 TP F

-Veh 1 TP M

$\square$ VPA Veh $2 \mathrm{~F}$

$\square$ VPA Veh2 M

$\square$ VPA TP F

-VPA TP M

Figure 3: Latency of behaviors from WRA (mean \pm SEM). A) wheel running by treatment group, B) contact with the novel mouse by treatment group, C) following novel mouse by treatment group. Note: * = first day the wheel was jammed. When the wheel is jammed, "wheel running" is defined as any activity that would make the wheel move if it were free. ${ }^{* *}$ Indicates statistically significant difference $(\mathrm{p}<0.05)$. 


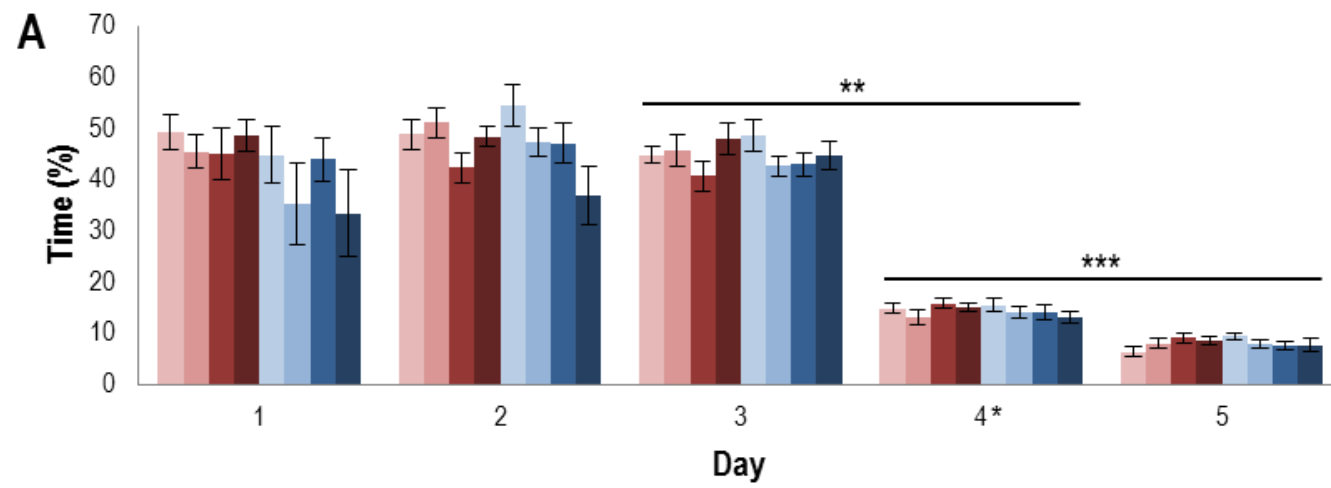

- Veh 1 Veh $2 \mathrm{~F}$

- Veh 1 Veh $2 \mathrm{M}$

-Veh 1 TP F

-Veh 1 TP M

$\square$ VPA Veh $2 \mathrm{~F}$

VPA Veh2 M

- VPA TP $F$

-VPA TP M

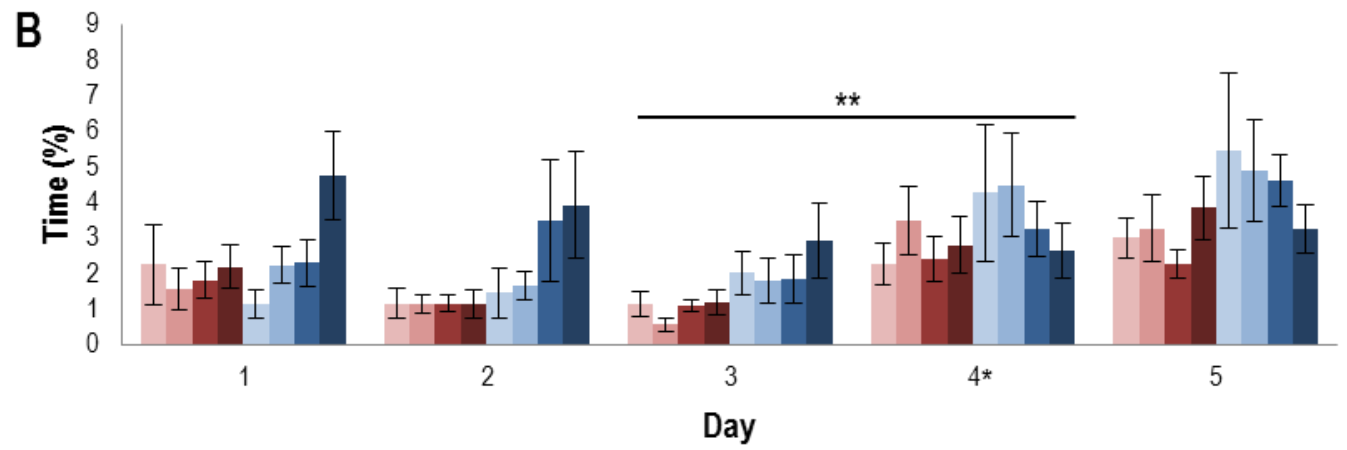

Veh 1 Veh $2 \mathrm{~F}$

- Veh 1 Veh $2 \mathrm{M}$

- Veh 1 TP F

- Veh 1 TP M

-VPA Veh $2 \mathrm{~F}$

- VPA Veh2 M

- VPA TP F

- VPA TP M
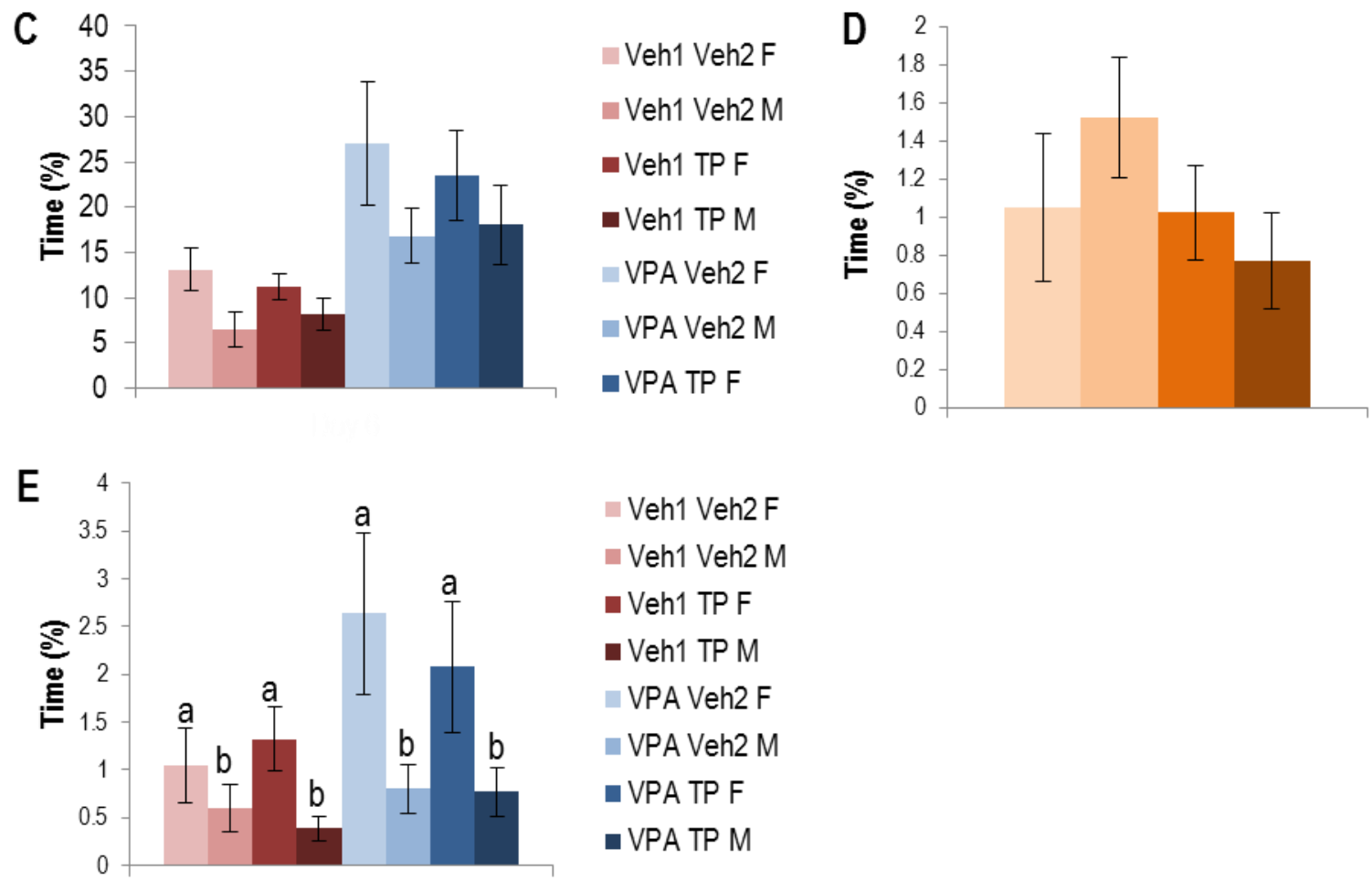

$$
\begin{aligned}
& \square \text { Veh1 Veh2 F } \\
& \text { Veh1 Veh2 M } \\
& \text { Veh1 TP F } \\
& \text { - Veh1 TP M } \\
& \square \text { VPA Veh2 F } \\
& \square \text { VPA Veh2 M } \\
& \square \text { VPA TP F } \\
& \square \text { VPA TP M }
\end{aligned}
$$

Figure 4: Duration of behaviors from WRA (mean $\% \pm$ SEM). A) wheel running by treatment group, B) grooming by treatment group, C) contact with the novel mouse by 
treatment group, D) following novel mouse by number of hits, E) following novel mouse by treatment group. Note: $*=$ first day the wheel was jammed. When the wheel is jammed, "wheel running" is defined as any activity that would make the wheel move if it were free. ${ }^{a b}$ Differing superscripts and ${ }^{* *}$ indicates statistically significant difference $(\mathrm{p}<$ $0.05)$. 


\section{CHAPTER 4}

\section{SUMMARY AND FUTURE DIRECTIONS}

\section{Summary}

This dissertation represents a compilation of work aimed at expanding our understanding of sex differences in autism incidence, using a mouse model to evaluate whether elevated levels of perinatal testosterone (pT) affect the vulnerability of each sex to an autism-inducing insult. In Chapter 1 , I discuss relevant literature on the sex bias in autism spectrum disorders, drawing on both clinical and animal models. Chapter 2 establishes baseline wheel running and social interaction behavior in pre-pubertal mice using an assay designed to measure autism-like behavioral deficits. These results suggest that there are no sex differences in wheel running behaviors or social interaction across 3 strains of mice. Findings from Chapter 3 support the premise that valproic acid (VPA) exposure impairs motor development abilities in mice and provides evidence that the combined effects of an autism-inducing insult (VPA), along with elevated pT (testosterone propionate, TP) and maleness (triple hit), results in worse performance on some motor development measures and increases cognitive rigidity and self-grooming behaviors compared to control females. This triple hit also led to some unexpected outcomes, such as reduced anxiety compared to other treatment groups. Finally, we found no associations between VPA treatment, $\mathrm{pT}$ treatment, or sex on cerebellar volume measures. Overall, these results offer limited support for the hypothesis that elevated 
levels of $\mathrm{pT}$ increases vulnerability to an autism-inducing insult. However, more research is needed in mice to

better validate this model and to elucidate the mechanism by which $\mathrm{pT}$ can increase susceptibility to autism-inducing insults, such as VPA.

\section{Future Directions}

As discussed in Chapter 3, prenatal treatment with VPA resulted in abnormal motor development, behaviors whose maturation are known to be associated with cerebellar development (Wagner et al., 2006). However, the effect of VPA on cerebellar development did not result in significant gross volumetric differences. In addition to associations with reduced Purkinje cell number and cerebellar volume (Ingram et al., 2000), VPA treatment in rodent models is linked to decreased neuroligin 3 mRNA expression (Kolozsi et al., 2009), increased corticosterone and decreased immunological activity (Schneider et al., 2008), and lower cortical expression of brain-derived neurotrophic factor (BDNF) mRNA (Roullet et al., 2010), all of which are relevant to autism spectrum disorders (ASD).

During the experiments outlined in Chapter 3, I reserved a small sample of 16 mice from cohort 3 for post-processing to allow for a gene expression pilot study. My lab is currently pursuing funding to extract total RNA from a hemi-cerebellum from each mouse for RNA-Seq assessment of differential gene expression among our treatment groups. As I have found no prior studies of gene expression in VPA rodent models which focus on the cerebellum $(\mathrm{Cb})$, RNA-Seq provides information on highly differentially expressed genes for targeted immunohistochemistry (IHC) follow up using our second series of sagittal brain sections from the 80 mice that underwent intracardial perfusion. 
Alternately, if the RNA-Seq pilot is not pursued, real-time PCR could be performed on cerebellar vermis and hemisphere samples. I propose five genes of interest (Bdnf, Dpp6, Galnt13, Astn2, and Ptchd1) because they are known to be associated with ASD and are expressed in the cerebellar cortex of both humans and mice (Menashe et al., 2013), and in the case of $B d n f$, because VPA decreases its expression in the mouse somatosensory cortex (Roullet et al., 2010) and steroid-induced BNDF is important for Purkinje cell development and connectivity in postnatal rodents (Tsutsui, 2008).

Given that the results from the hits analyses suggest that $\mathrm{pT}$ could be acting to increase vulnerability of males to our autism-inducing insult, further studies designed to understand the mechanisms of testosterone (T) action that increase susceptibility to VPA treatment may be warranted. Recent studies investigating postnatal $\mathrm{Cb}$ steroidogenesis and development, but without an autism-inducing component, point to possible next steps to generate hypotheses to investigate mechanisms. The $\mathrm{Cb}$ develops later than many other brain regions, making it particularly vulnerable to postnatal perturbations (Dean \& McCarthy, 2008). The rodent $\mathrm{Cb}$ has LH-dependent de novo neurosteroidogenesis and all pathway components to get from cholesterol to 17ß-estradiol (E2) (E1 Hajj Chehadeh et al., 2014); the $\mathrm{Cb}$ expresses both estrogen receptor subtypes throughout postnatal development, but androgen receptors appear later, at some point between P7 and P20 (Qin et al., 2007), suggesting that early $\mathrm{T}$ effects on $\mathrm{Cb}$ are mediated by its metabolite $\mathrm{E} 2$. Postnatal male rats produce a spike in mRNA for steroidogenic pathway components (StAR protein, $\mathrm{p} 450 \mathrm{scc}$, and aromatase) at P10, with females producing significantly less than males and their aromatase mRNA spike occurring earlier, at P5 (Lavaque, Mayen, Azcoitia, Tena-Sempere, \& Garcia-Segura, 2006). Injecting female rats at P1 with 
$12.5 \mathrm{mg} /$ rat $\mathrm{TP}$ to mimic the postnatal gonadal $\mathrm{T}$ surge in males did not alter their $\mathrm{Cb}$ steroid pathway mRNA levels at P10 compared to oil-injected females (Lavaque et al., 2006), but $\mathrm{Cb}$ steroid levels were not measured. This result indicates that effects of $\mathrm{pT}$ are probably not via its effects on de novo steroidogenesis in the $\mathrm{Cb}$, or if they are, then a previous insult (such as VPA) is also necessary. Allopregnanolone, progesterone, and E2 from Purkinje cells organize $\mathrm{Cb}$ maturation, in part by stimulating BDNF expression, which is necessary for postnatal $\mathrm{Cb}$ maturation and connectivity; aromatase knockout (ArKO) mice express decreased $B d n f$ in $\mathrm{Cb}$ and decreased Purkinje cell maturation compared to wild types or ArKO mice given supplemental estrogen neonatally (Tsutsui, 2008), pointing to a beneficial role of locally-produced $\mathrm{E} 2$. Therefore, $\mathrm{Cb}$ neurosteroidogenesis is a logical next step to pursue. A recent study in rats demonstrates that overstimulation with E2 may be detrimental; prostaglandin-stimulated E2 production in the $\mathrm{Cb}$ at P10-12 stunted Purkinje cell dendritic arbors and altered their electrophysiological properties in both males and females (Dean, Knutson, Krebs-Kraft, \& McCarthy, 2012). Perhaps extra-cerebellar T (gonadal or exogenous), acting as E2 on a VPA-disordered brain, overwhelms normal processes of $\mathrm{Cb}$ development, leading to increased autism-like effects in triple hit males compared to control females.

The proposed studies will provide information on gene expression and help further elucidate mechanisms of testosterone action that increase susceptibility to VPA treatment. The results of the current experiments, while not fully supporting our hypotheses, provide some evidence that elevated levels of $\mathrm{pT}$ increase vulnerability to an autism-inducing insult in our mouse model. This warrants further study using this VPATP model with the caveat that more data is needed to identify the appropriate timing, 
dose, and delivery method of VPA and TP to attain a reliable model of ASD. The sex bias in ASD remains one of the hallmark features of this condition, and undoubtedly a better understanding of this difference in incidence can provide much needed information about the underlying causes. 


\section{REFERENCES}

Albert, M., and Roussel, C. (1984). Strain differences in the concentration, motility and morphology of epididymal sperm in relation to puberty in mice. International Journal of Andrology, 7, 34-37.

Alycia K. Halladay, S. B., John N. Constantino, Amy M. Daniels, Katheen Koenig, Kate Palmer, Daniel Messinger, Kevin Pelphrey, Stephan J. Sanders, Alison Tepper Singer, Julie Lounds Taylor, and Peter Szatmari. (2015). Sex and gender differences in autism spectrum disorder: summarizing evidence gaps and identifying emerging areas of priority. Mol Autism, 6(36). doi:10.1186/s13229015-0019-y

Amaral, D. G., Schumann, C. M., \& Nordahl, C. W. (2008). Neuroanatomy of autism. Trends Neurosci, 31(3), 137-145. doi:10.1016/j.tins.2007.12.005

Amateau, S. K., \& McCarthy, M. M. (2002). A novel mechanism of dendritic spine plasticity involving estradiol induction of prostaglandin-E2. J Neurosci, 22(19), 8586-8596. Retrieved from http://www.ncbi.nlm.nih.gov/pubmed/12351732

Anello, A., Reichenberg, A., Luo, X., Schmeidler, J., Hollander, E., Smith, C. J., . . Silverman, J. M. (2009). Brief report: parental age and the sex ratio in autism. $J$ Autism Dev Disord, 39(10), 1487-1492. doi:10.1007/s10803-009-0755-y

Asperger, H. (1944). The "autistic psychopathy" in childhood. Archiv Fur Psychiatrie Und Nervenkrankheiten, 117(1), 76-136. 
Atladottir, H. O., Thorsen, P., Ostergaard, L., Schendel, D. E., Lemcke, S., Abdallah, M., \& Parner, E. T. (2010). Maternal infection requiring hospitalization during pregnancy and autism spectrum disorders. J Autism Dev Disord, 40(12), 14231430. doi:10.1007/s10803-010-1006-y

Auyeung, B., Ahluwalia, J., Thomson, L., Taylor, K., Hackett, G., O'Donnell, K. J., \& Baron-Cohen, S. (2012). Prenatal versus postnatal sex steroid hormone effects on autistic traits in children at 18 to 24 months of age. Mol Autism, 3(1), 17. doi:10.1186/2040-2392-3-17

Auyeung, B., Taylor, K., Hackett, G., \& Baron-Cohen, S. (2010). Foetal testosterone and autistic traits in 18 to 24-month-old children. Mol Autism, 1(1), 11. doi:10.1186/2040-2392-1-11

Baker, K. B., Wray, S. P., Ritter, R., Mason, S., Lanthorn, T. H., \& Savelieva, K. V. (2010). Male and female Fmr1 knockout mice on C57 albino background exhibit spatial learning and memory impairments. Genes Brain Behav, 9(6), 562-574. doi:10.1111/j.1601-183X.2010.00585.x

Bao, A. M., \& Swaab, D. F. (2011). Sexual differentiation of the human brain: relation to gender identity, sexual orientation and neuropsychiatric disorders. Front Neuroendocrinol, 32(2), 214-226. doi:10.1016/j.yfrne.2011.02.007

Baron-Cohen, S., Auyeung, B., Norgaard-Pedersen, B., Hougaard, D. M., Abdallah, M. W., Melgaard, L., . . Lombardo, M. V. (2014). Elevated fetal steroidogenic activity in autism. Mol Psychiatry. doi:10.1038/mp.2014.48 
Baron-Cohen, S., Knickmeyer, R. C., \& Belmonte, M. K. (2005). Sex differences in the brain: implications for explaining autism. Science, 310(5749), 819-823. doi: $10.1126 /$ science. 1115455

Baron-Cohen, S., Lombardo, M. V., Auyeung, B., Ashwin, E., Chakrabarti, B., \& Knickmeyer, R. (2011). Why are autism spectrum conditions more prevalent in males? PLoS Biol, 9(6), e1001081. doi:10.1371/journal.pbio.1001081

Beacher, F. D., Minati, L., Baron-Cohen, S., Lombardo, M. V., Lai, M. C., Gray, M. A., . . Critchley, H. D. (2012). Autism attenuates sex differences in brain structure: a combined voxel-based morphometry and diffusion tensor imaging study. AJNR Am J Neuroradiol, 33(1), 83-89. doi:10.3174/ajnr.A2880

Beatty, W. W. (1992). Gonadal hormones and sex differences in nonreporductive behaviors. In A. A. M. Gerall, Howard; and Ward, Ingeborg L. (Ed.), Sexual Differentiation (Vol. 11, pp. 85-128). New York: Plenum Press.

Beaudet, A. L. a. Z., Huda Y. (2006). A mixed epigenetic and genetic and mixed de novo and inherited model for autism. In S. O. a. R. Moldin, John. L.R. (Ed.), Understanding autism: from basic nuroscience to treatment (pp. 95-111). Boca Raton: Taylor \& Francis Group, LLC.

Becker, J. B., Arnold, A. P., Berkley, K. J., Blaustein, J. D., Eckel, L. A., Hampson, E., . . . Young, E. (2005). Strategies and methods for research on sex differences in brain and behavior. Endocrinology, 146(4), 1650-1673. doi:10.1210/en.20041142

Beery, A. K., \& Zucker, I. (2011). Sex bias in neuroscience and biomedical research. Neurosci Biobehav Rev, 35(3), 565-572. doi:10.1016/j.neubiorev.2010.07.002 
Ben-Itzchak, E., Ben-Shachar, S., \& Zachor, D. A. (2013). Specific neurological phenotypes in autism spectrum disorders are associated with sex representation. Autism Res, 6(6), 596-604. doi:10.1002/aur.1319

Bishop, D. V., Jacobs, P. A., Lachlan, K., Wellesley, D., Barnicoat, A., Boyd, P. A., . . . Scerif, G. (2011). Autism, language and communication in children with sex chromosome trisomies. Arch Dis Child, 96(10), 954-959. doi:10.1136/adc.2009.179747

Bloss, C. S., \& Courchesne, E. (2007). MRI neuroanatomy in young girls with autism: a preliminary study. J Am Acad Child Adolesc Psychiatry, 46(4), 515-523. doi:10.1097/chi.0b013e318030e28b

Bolivar, V. J., Walters, S. R., \& Phoenix, J. L. (2007). Assessing autism-like behavior in mice: variations in social interactions among inbred strains. Behav Brain Res, 176(1), 21-26. doi:10.1016/j.bbr.2006.09.007

Bowen, R. S., Knab, A. M., Hamilton, A. T., McCall, J. R., Moore-Harrison, T. L., \& Lightfoot, J. T. (2012). Effects of supraphysiological doses of sex steroids on wheel running activity in mice. J Steroids Horm Sci, 3(2), 110. doi:10.4172/21577536.1000110

Broida, J., \& Svare, B. (1984). Sex differences in the activity of mice: modulation by postnatal gonadal hormones. Horm Behav, 18(1), 65-78. Retrieved from http://www.ncbi.nlm.nih.gov/pubmed/6706320

Cahill, L. (2012). A half-truth is a whole lie: on the necessity of investigating sex influences on the brain. Endocrinology, 153(6), 2541-2543. doi:10.1210/en.20112167 
Carter, A. S., Black, D. O., Tewani, S., Connolly, C. E., Kadlec, M. B., \& TagerFlusberg, H. (2007). Sex differences in toddlers with autism spectrum disorders. $J$ Autism Dev Disord, 37(1), 86-97. doi:10.1007/s10803-006-0331-7

Casanova, M. F. (2007). The neuropathology of autism. Brain Pathol, 17(4), 422-433. doi:10.1111/j.1750-3639.2007.00100.x

Center for Disease Control and Prevention. (2016, February 26, 2015). Autism Spectrum Disorder Data and Statistics.

Chapman, E., Baron-Cohen, S., Auyeung, B., Knickmeyer, R., Taylor, K., \& Hackett, G. (2006). Fetal testosterone and empathy: evidence from the empathy quotient (EQ) and the "reading the mind in the eyes" test. Soc Neurosci, 1(2), 135-148. doi:10.1080/17470910600992239

Charles River Laboratories International Inc. (2014a). CF-1 ${ }^{\mathrm{TM}}$ Mouse Growth Chart. Retrieved from http://www.criver.com/products-services/basic-research/find-a$\underline{\text { model/cf-1-mouse }}$

Charles River Laboratories International Inc. (2014b). Swiss Webster (CFW®) Growth Chart Retrieved from http://www.criver.com/products-services/basicresearch/find-a-model/swiss-webster-\%28cfw\%29-mouse. Retrieved 08/14/2014 http://www.criver.com/products-services/basic-research/find-a-model/swisswebster-\%28cfw\%29-mouse

Chess, S. (1977). Follow-up report on autism in congenital rubella. J Autism Child Schizophr, 7(1), 69-81. Retrieved from http://www.ncbi.nlm.nih.gov/pubmed/576606 
Chess, S., Fernandez, P., \& Korn, S. (1978). Behavioral consequences of congenital rubella. J Pediatr, 93(4), 699-703. Retrieved from http://www.ncbi.nlm.nih.gov/pubmed/702254

Chih, B., Afridi, S. K., Clark, L., \& Scheiffele, P. (2004). Disorder-associated mutations lead to functional inactivation of neuroligins. Hum Mol Genet, 13(14), 1471-1477. doi:10.1093/hmg/ddh158

Christensen, J., Gronborg, T. K., Sorensen, M. J., Schendel, D., Parner, E. T., Pedersen, L. H., \& Vestergaard, M. (2013). Prenatal valproate exposure and risk of autism spectrum disorders and childhood autism. JAMA, 309(16), 1696-1703. doi:10.1001/jama.2013.2270

Cohly, H. H., \& Panja, A. (2005). Immunological findings in autism. Int Rev Neurobiol, 71,317-341. Retrieved from http://www.ncbi.nlm.nih.gov/pubmed/16512356

Colledge, W. H., Mei, H., \& d'Anglemont de Tassigny, X. (2010). Mouse models to study the central regulation of puberty. Mol Cell Endocrinol, 324(1-2), 12-20. doi:10.1016/j.mce.2010.01.015

Comoletti, D., De Jaco, A., Jennings, L. L., Flynn, R. E., Gaietta, G., Tsigelny, I., . . . Taylor, P. (2004). The Arg451Cys-neuroligin-3 mutation associated with autism reveals a defect in protein processing. J Neurosci, 24(20), 4889-4893. doi:10.1523/JNEUROSCI.0468-04.2004

Courchesne, E., Karns, C. M., Davis, H. R., Ziccardi, R., Carper, R. A., Tigue, Z. D., . . . Courchesne, R. Y. (2001). Unusual brain growth patterns in early life in patients with autistic disorder: an MRI study. Neurology, 57(2), 245-254. Retrieved from http://www.ncbi.nlm.nih.gov/pubmed/11468308 
Crawley, J. N. (2007). Mouse behavioral assays relevant to the symptoms of autism. Brain Pathol, 17(4), 448-459. doi:10.1111/j.1750-3639.2007.00096.x

Creswell, C. S., DH. (1999). Autism in association with Turner syndrome: Genetic implications for male vulnerability to pervasive developmental disorders. Neurocase, 5(6), 511-518.

Croen, L. A., Najjar, D. V., Fireman, B., \& Grether, J. K. (2007). Maternal and paternal age and risk of autism spectrum disorders. Arch Pediatr Adolesc Med, 161(4), 334-340. doi:10.1001/archpedi.161.4.334

Davis, E. P., \& Pfaff, D. (2014). Sexually dimorphic responses to early adversity: implications for affective problems and autism spectrum disorder. Psychoneuroendocrinology, 49, 11-25. doi:10.1016/j.psyneuen.2014.06.014

De Rubeis, S., \& Buxbaum, J. D. (2015). Genetics and genomics of autism spectrum disorder: embracing complexity. Hum Mol Genet. doi:10.1093/hmg/ddv273

Dean, S. L., Knutson, J. F., Krebs-Kraft, D. L., \& McCarthy, M. M. (2012). Prostaglandin E2 is an endogenous modulator of cerebellar development and complex behavior during a sensitive postnatal period. Eur J Neurosci, 35(8), 1218-1229. doi:10.1111/j.1460-9568.2012.08032.x

Dean, S. L., \& McCarthy, M. M. (2008). Steroids, sex and the cerebellar cortex: implications for human disease. Cerebellum, 7(1), 38-47. doi:10.1007/s12311008-0003-6

Diagnostic and Statistical Manual of Mental Disorders. (2013). Washington, D.C.: American Psychiatric Association. 
Ding, Q., Sethna, F., \& Wang, H. (2014). Behavioral analysis of male and female Fmr1 knockout mice on C57BL/6 background. Behav Brain Res, 271, 72-78. doi:10.1016/j.bbr.2014.05.046

Donnelly, S. L., Wolpert, C. M., Menold, M. M., Bass, M. P., Gilbert, J. R., Cuccaro, M. L., ... Pericak-Vance, M. A. (2000). Female with autistic disorder and monosomy X (Turner syndrome): parent-of-origin effect of the X chromosome. Am J Med Genet, 96(3), 312-316. Retrieved from http://www.ncbi.nlm.nih.gov/pubmed/10898907

Dowse, H., Umemori, J., \& Koide, T. (2010). Ultradian components in the locomotor activity rhythms of the genetically normal mouse, Mus musculus. $J$ Exp Biol, 213(Pt 10), 1788-1795. doi:10.1242/jeb.038877

Dufour-Rainfray, D., Vourc'h, P., Le Guisquet, A. M., Garreau, L., Ternant, D., Bodard, S., .. . Guilloteau, D. (2010). Behavior and serotonergic disorders in rats exposed prenatally to valproate: a model for autism. Neurosci Lett, 470(1), 55-59. doi:10.1016/j.neulet.2009.12.054

Durkin, M. S., Maenner, M. J., Newschaffer, C. J., Lee, L. C., Cunniff, C. M., Daniels, J. L., ... Schieve, L. A. (2008). Advanced parental age and the risk of autism spectrum disorder. Am J Epidemiol, 168(11), 1268-1276. doi:10.1093/aje/kwn250 Dworzynski, K., Ronald, A., Bolton, P., \& Happe, F. (2012). How different are girls and boys above and below the diagnostic threshold for autism spectrum disorders? $J$ Am Acad Child Adolesc Psychiatry, 51(8), 788-797. doi:10.1016/j.jaac.2012.05.018 
El Hajj Chehadeh, S., Dreumont, N., Willekens, J., Canabady-Rochelle, L., Jeannesson, E., Alberto, J. M., . . Leininger-Muller, B. (2014). Early methyl donor deficiency alters cAMP signaling pathway and neurosteroidogenesis in the cerebellum of female rat pups. Am J Physiol Endocrinol Metab, 307(11), E1009-1019. doi:10.1152/ajpendo.00364.2014

Emack, J., Kostaki, A., Walker, C. D., \& Matthews, S. G. (2008). Chronic maternal stress affects growth, behaviour and hypothalamo-pituitary-adrenal function in juvenile offspring. Horm Behav, 54(4), 514-520. doi:10.1016/j.yhbeh.2008.02.025

Evans, J. S., Varney, Roger F., and Koch, F. C. (1941). The mouse uterine weight method for the assay of estrogens. Endocrinology, 28(5), 747-752.

Fombonne, E. (2003). Epidemiological surveys of autism and other pervasive developmental disorders: an update. J Autism Dev Disord, 33(4), 365-382. Retrieved from http://www.ncbi.nlm.nih.gov/pubmed/12959416

Fombonne, E. (2005). The Changing Epidemiology of Autism. Journal of Applied Research in Intellectual Disabilities, 18, 281-294.

Fombonne, E. (2009). Epidemiology of pervasive developmental disorders. Pediatr Res, 65(6), 591-598. doi:10.1203/PDR.0b013e31819e7203

Fournier, K. A., Hass, C. J., Naik, S. K., Lodha, N., \& Cauraugh, J. H. (2010). Motor coordination in autism spectrum disorders: a synthesis and meta-analysis. $J$ Autism Dev Disord, 40(10), 1227-1240. doi:10.1007/s10803-010-0981-3

Franklin, T. B., Russig, H., Weiss, I. C., Graff, J., Linder, N., Michalon, A., . . Mansuy, I. M. (2010). Epigenetic transmission of the impact of early stress across generations. Biol Psychiatry, 68(5), 408-415. doi:10.1016/j.biopsych.2010.05.036 
Gandal, M. J., Edgar, J. C., Ehrlichman, R. S., Mehta, M., Roberts, T. P., \& Siegel, S. J. (2010). Validating gamma oscillations and delayed auditory responses as translational biomarkers of autism. Biol Psychiatry, 68(12), 1100-1106. doi:10.1016/j.biopsych.2010.09.031

Garland, T., Jr., Kelly, S. A., Malisch, J. L., Kolb, E. M., Hannon, R. M., Keeney, B. K., . . Middleton, K. M. (2011). How to run far: multiple solutions and sex-specific responses to selective breeding for high voluntary activity levels. Proc Biol Sci, 278(1705), 574-581. doi:10.1098/rspb.2010.1584

Geier, D. A., \& Geier, M. R. (2006). A clinical and laboratory evaluation of methionine cycle-transsulfuration and androgen pathway markers in children with autistic disorders. Horm Res, 66(4), 182-188. doi:10.1159/000094467

Geier, D. A., \& Geier, M. R. (2007). A prospective assessment of androgen levels in patients with autistic spectrum disorders: biochemical underpinnings and suggested therapies. Neuro Endocrinol Lett, 28(5), 565-573. Retrieved from http://www.ncbi.nlm.nih.gov/pubmed/17984958

Geschwind, D. H., Boone, K. B., Miller, B. L., \& Swerdloff, R. S. (2000). Neurobehavioral phenotype of Klinefelter syndrome. Ment Retard Dev Disabil Res Rev, 6(2), 107-116. doi:10.1002/1098-2779(2000)6:2<107::AIDMRDD4>3.0.CO;2-2

Giraldez-Perez, R. M., Avila, M. N., Feijoo-Cuaresma, M., Heredia, R., De Diego-Otero, Y., Real, M. A., \& Guirado, S. (2013). Males but not females show differences in calbindin immunoreactivity in the dorsal thalamus of the mouse model of fragile X syndrome. J Comp Neurol, 521(4), 894-911. doi:10.1002/cne.23209 
Glaze, D. G. (2004). Rett syndrome: of girls and mice--lessons for regression in autism. Ment Retard Dev Disabil Res Rev, 10(2), 154-158. doi:10.1002/mrdd.20030

Gordon, E., \& Corbitt, C. (2015). Investigation of prepubertal sex differences in wheel running and social behavior in three mouse strains. Journal of Ethology, 33(3), 177-187. doi:10.1007/s10164-015-0429-5

Gore, A. C., Martien, K. M., Gagnidze, K., \& Pfaff, D. (2014). Implications of prenatal steroid perturbations for neurodevelopment, behavior, and autism. Endocr Rev, 35(6), 961-991. doi:10.1210/er.2013-1122

Guide for the Care and Use of Laboratory Animals. (2011). Washington, D.C.: The National Academies Press

Gundersen, H. J., Jensen, E. B., Kieu, K., \& Nielsen, J. (1999). The efficiency of systematic sampling in stereology--reconsidered. J Microsc, 193(Pt 3), 199-211.

Hallmayer, J., Cleveland, S., Torres, A., Phillips, J., Cohen, B., Torigoe, T., . . Risch, N. (2011). Genetic heritability and shared environmental factors among twin pairs with autism. Arch Gen Psychiatry, 68(11), 1095-1102. doi:10.1001/archgenpsychiatry.2011.76

Hara, Y., Maeda, Y., Kataoka, S., Ago, Y., Takuma, K., \& Matsuda, T. (2012). Effect of prenatal valproic acid exposure on cortical morphology in female mice. $J$ Pharmacol Sci, 118(4), 543-546. Retrieved from http://www.ncbi.nlm.nih.gov/pubmed/22447305

Hartley, S. L., \& Sikora, D. M. (2009). Sex differences in autism spectrum disorder: an examination of developmental functioning, autistic symptoms, and coexisting 
behavior problems in toddlers. J Autism Dev Disord, 39(12), 1715-1722. doi:10.1007/s10803-009-0810-8

Hashimoto, T., Tayama, M., Murakawa, K., Yoshimoto, T., Miyazaki, M., Harada, M., \& Kuroda, Y. (1995). Development of the brainstem and cerebellum in autistic patients. J Autism Dev Disord, 25(1), 1-18. Retrieved from http://www.ncbi.nlm.nih.gov/pubmed/7608030

Hiller, R. M., Young, R. L., \& Weber, N. (2014). Sex differences in autism spectrum disorder based on DSM-5 criteria: evidence from clinician and teacher reporting. J Abnorm Child Psychol, 42(8), 1381-1393. doi:10.1007/s10802-014-9881-x

Hines, M. (2006). Prenatal testosterone and gender-related behaviour. Eur J Endocrinol, 155 Suppl 1, S115-121. doi:10.1530/eje.1.02236

Hines, M., Fane, B. A., Pasterski, V. L., Mathews, G. A., Conway, G. S., \& Brook, C. (2003). Spatial abilities following prenatal androgen abnormality: targeting and mental rotations performance in individuals with congenital adrenal hyperplasia. Psychoneuroendocrinology, 28(8), 1010-1026. Retrieved from http://www.ncbi.nlm.nih.gov/pubmed/14529705

Hines, M., \& Kaufman, F. R. (1994). Androgen and the development of human sextypical behavior: rough-and-tumble play and sex of preferred playmates in children with congenital adrenal hyperplasia (CAH). Child Dev, 65(4), 10421053. Retrieved from http://www.ncbi.nlm.nih.gov/pubmed/7956464

Holtmann, M., Bolte, S., \& Poustka, F. (2007). Autism spectrum disorders: sex differences in autistic behaviour domains and coexisting psychopathology. Dev Med Child Neurol, 49(5), 361-366. doi:10.1111/j.1469-8749.2007.00361.x 
Idring, S., Lundberg, M., Sturm, H., Dalman, C., Gumpert, C., Rai, D., Lee, B.K., and Magnusson, C. (2014). Changes in Prevalence of Autism Spectrum Disorders in 2001-2011: Findings from the Stockholm Youth Cohort. J Autism Dev Disord, 45(6), 1766-1773.

Ingram, J. L., Peckham, S. M., Tisdale, B., \& Rodier, P. M. (2000). Prenatal exposure of rats to valproic acid reproduces the cerebellar anomalies associated with autism. Neurotoxicol Teratol, 22(3), 319-324. Retrieved from http://www.ncbi.nlm.nih.gov/pubmed/10840175

Ingudomnukul, E., Baron-Cohen, S., Wheelwright, S., \& Knickmeyer, R. (2007). Elevated rates of testosterone-related disorders in women with autism spectrum conditions. Horm Behav, 51(5), 597-604. doi:10.1016/j.yhbeh.2007.02.001

Jamain, S., Quach, H., Betancur, C., Rastam, M., Colineaux, C., Gillberg, I. C., . . Paris Autism Research International Sibpair, S. (2003). Mutations of the X-linked genes encoding neuroligins NLGN3 and NLGN4 are associated with autism. Nat Genet, 34(1), 27-29. doi:10.1038/ng1136

Jamain, S., Quach, H., Quintana-Murci, L., Betancur, C., Philippe, A., Gillberg, C., . . . Bourgeron, T. (2002). Y chromosome haplogroups in autistic subjects. Mol Psychiatry, 7(2), 217-219. doi:10.1038/sj.mp.4000968

Kanner, L. (1943). Autistic disturbances of affective contact. Nervous Child, 2(3), 217250.

Karvat, G., \& Kimchi, T. (2012). Systematic autistic-like behavioral phenotyping of 4 mouse strains using a novel wheel-running assay. Behav Brain Res, 233(2), 405414. doi:10.1016/j.bbr.2012.05.028 
Kataoka, S., Takuma, K., Hara, Y., Maeda, Y., Ago, Y., \& Matsuda, T. (2013). Autismlike behaviours with transient histone hyperacetylation in mice treated prenatally with valproic acid. Int J Neuropsychopharmacol, 16(1), 91-103. doi:10.1017/S1461145711001714

Kelly, S. J., Ostrowski, N. L., \& Wilson, M. A. (1999). Gender differences in brain and behavior: hormonal and neural bases. Pharmacol Biochem Behav, 64(4), 655-664. Retrieved from http://www.ncbi.nlm.nih.gov/pubmed/10593187

Kent, S., Hurd, M., \& Satinoff, E. (1991). Interactions between body temperature and wheel running over the estrous cycle in rats. Physiol Behav, 49(6), 1079-1084. Retrieved from http://www.ncbi.nlm.nih.gov/pubmed/1896490

Kim, K. C., Kim, P., Go, H. S., Choi, C. S., Yang, S. I., Cheong, J. H., . . Ko, K. H. (2011). The critical period of valproate exposure to induce autistic symptoms in Sprague-Dawley rats. Toxicol Lett, 201(2), 137-142.

doi:10.1016/j.toxlet.2010.12.018

Kim, Y. S., Leventhal, B. L., Koh, Y. J., Fombonne, E., Laska, E., Lim, E. C., . . Grinker, R. R. (2011). Prevalence of autism spectrum disorders in a total population sample. Am J Psychiatry, 168(9), 904-912. doi:10.1176/appi.ajp.2011.10101532

Knickmeyer, R., Baron-Cohen, S., Fane, B. A., Wheelwright, S., Mathews, G. A., Conway, G. S., . . . Hines, M. (2006). Androgens and autistic traits: A study of individuals with congenital adrenal hyperplasia. Horm Behav, 50(1), 148-153. doi:10.1016/j.yhbeh.2006.02.006 
Knickmeyer, R., Baron-Cohen, S., Raggatt, P., \& Taylor, K. (2005). Foetal testosterone, social relationships, and restricted interests in children. J Child Psychol Psychiatry, 46(2), 198-210. doi:10.1111/j.1469-7610.2004.00349.x

Kohane, I. S., McMurry, A., Weber, G., MacFadden, D., Rappaport, L., Kunkel, L., . . . Churchill, S. (2012). The co-morbidity burden of children and young adults with autism spectrum disorders. PLoS One, 7(4), e33224. doi:10.1371/journal.pone.0033224

Kolozsi, E., Mackenzie, R. N., Roullet, F. I., deCatanzaro, D., \& Foster, J. A. (2009). Prenatal exposure to valproic acid leads to reduced expression of synaptic adhesion molecule neuroligin 3 in mice. Neuroscience, 163(4), 1201-1210. doi:10.1016/j.neuroscience.2009.07.021

Kreiser, N. L., \& White, S. W. (2014). ASD in females: are we overstating the gender difference in diagnosis? Clin Child Fam Psychol Rev, 17(1), 67-84. doi:10.1007/s10567-013-0148-9

Lai, D. C., Tseng, Y. C., Hou, Y. M., \& Guo, H. R. (2012). Gender and geographic differences in the prevalence of autism spectrum disorders in children: analysis of data from the national disability registry of Taiwan. Res Dev Disabil, 33(3), 909915. doi:10.1016/j.ridd.2011.12.015

Lai, M. C., Baron-Cohen, S., \& Buxbaum, J. D. (2015). Understanding autism in the light of sex/gender. Mol Autism, 6, 24. doi:10.1186/s13229-015-0021-4

Lai, M. C., Lombardo, M. V., Auyeung, B., Chakrabarti, B., \& Baron-Cohen, S. (2015). Sex/gender differences and autism: setting the scene for future research. $J \mathrm{Am}$ Acad Child Adolesc Psychiatry, 54(1), 11-24. doi:10.1016/j.jaac.2014.10.003 
Lai, M. C., Lombardo, M. V., Pasco, G., Ruigrok, A. N., Wheelwright, S. J., Sadek, S. A., ... Baron-Cohen, S. (2011). A behavioral comparison of male and female adults with high functioning autism spectrum conditions. PLoS One, 6(6), e20835. doi:10.1371/journal.pone.0020835

Lai, M. C., Lombardo, M. V., Suckling, J., Ruigrok, A. N., Chakrabarti, B., Ecker, C., . . . Baron-Cohen, S. (2013). Biological sex affects the neurobiology of autism. Brain, 136(Pt 9), 2799-2815. doi:10.1093/brain/awt216

Lamberty, Y., \& Gower, A. J. (1988). Investigation into sex-related differences in locomotor activity, place learning and passive avoidance responding in NMRI mice. Physiol Behav, 44(6), 787-790. Retrieved from http://www.ncbi.nlm.nih.gov/pubmed/3249753

Laumonnier, F., Bonnet-Brilhault, F., Gomot, M., Blanc, R., David, A., Moizard, M. P., . .. Briault, S. (2004). X-linked mental retardation and autism are associated with a mutation in the NLGN4 gene, a member of the neuroligin family. Am J Hum Genet, 74(3), 552-557. doi:10.1086/382137

Lavaque, E., Mayen, A., Azcoitia, I., Tena-Sempere, M., \& Garcia-Segura, L. M. (2006). Sex differences, developmental changes, response to injury and cAMP regulation of the mRNA levels of steroidogenic acute regulatory protein, cytochrome p450scc, and aromatase in the olivocerebellar system. J Neurobiol, 66(3), 308318. doi:10.1002/neu.20221

Lenz, K. M., Nugent, B. M., Haliyur, R., \& McCarthy, M. M. (2013). Microglia are essential to masculinization of brain and behavior. J Neurosci, 33(7), 2761-2772. doi:10.1523/JNEUROSCI.1268-12.2013 
Lenz, K. M., Nugent, B. M., \& McCarthy, M. M. (2012). Sexual differentiation of the rodent brain: dogma and beyond. Front Neurosci, 6, 26. doi:10.3389/fnins.2012.00026

Limperopoulos, C., Chilingaryan, G., Sullivan, N., Guizard, N., Robertson, R. L., \& du Plessis, A. J. (2014). Injury to the premature cerebellum: outcome is related to remote cortical development. Cereb Cortex, 24(3), 728-736. doi:10.1093/cercor/bhs354

Lindsay, K. A., \& Widiger, T. A. (1995). Sex and gender bias in self-report personality disorder inventories: item analysis of the MCMI-II, MMPI, and PDQ-R. J Pers Assess, 65(1), 1-20. doi:10.1207/s15327752jpa6501_1

Lord, C., \& Schopler, E. (1985). Differences in sex ratios in autism as a function of measured intelligence. J Autism Dev Disord, 15(2), 185-193. Retrieved from http://www.ncbi.nlm.nih.gov/pubmed/3997745

Lord, C., Schopler, E., \& Revicki, D. (1982). Sex differences in autism. J Autism Dev Disord, 12(4), 317-330. Retrieved from http://www.ncbi.nlm.nih.gov/pubmed/7161234

Loscher, W. (1999). Valproate: a reappraisal of its pharmacodynamic properties and mechanisms of action. Prog Neurobiol, 58(1), 31-59. Retrieved from http://www.ncbi.nlm.nih.gov/pubmed/10321796

Mandy, W., Chilvers, R., Chowdhury, U., Salter, G., Seigal, A., \& Skuse, D. (2012). Sex differences in autism spectrum disorder: evidence from a large sample of children and adolescents. J Autism Dev Disord, 42(7), 1304-1313. doi:10.1007/s10803011-1356-0 
Markham, J. A., Beckel-Mitchener, A. C., Estrada, C. M., \& Greenough, W. T. (2006). Corticosterone response to acute stress in a mouse model of Fragile X syndrome. Psychoneuroendocrinology, 31(6), 781-785. doi:10.1016/j.psyneuen.2006.02.008

Markham, J. A., Taylor, A. R., Taylor, S. B., Bell, D. B., \& Koenig, J. I. (2010). Characterization of the cognitive impairments induced by prenatal exposure to stress in the rat. Front Behav Neurosci, 4, 173. doi:10.3389/fnbeh.2010.00173

Mattila, M. L., Kielinen, M., Linna, S. L., Jussila, K., Ebeling, H., Bloigu, R., . . . Moilanen, I. (2011). Autism spectrum disorders according to DSM-IV-TR and comparison with DSM-5 draft criteria: an epidemiological study. J Am Acad Child Adolesc Psychiatry, 50(6), 583-592 e511. doi:10.1016/j.jaac.2011.04.001 Mayer, C., Acosta-Martinez, M., Dubois, S. L., Wolfe, A., Radovick, S., Boehm, U., \& Levine, J. E. (2010). Timing and completion of puberty in female mice depend on estrogen receptor alpha-signaling in kisspeptin neurons. Proc Natl Acad Sci U S A, 107(52), 22693-22698. doi:10.1073/pnas.1012406108

McCarthy, M. M., Arnold, A. P., Ball, G. F., Blaustein, J. D., \& De Vries, G. J. (2012). Sex differences in the brain: the not so inconvenient truth. J Neurosci, 32(7), 2241-2247. doi:10.1523/JNEUROSCI.5372-11.2012

McCauley, E., Ito, J., \& Kay, T. (1986). Psychosocial functioning in girls with Turner's syndrome and short stature: social skills, behavior problems, and self-concept. $J$ Am Acad Child Psychiatry, 25(1), 105-112. Retrieved from http://www.ncbi.nlm.nih.gov/pubmed/3950258 
McGuire, L. S., Ryan, K. O., \& Omenn, G. S. (1975). Congenital adrenal hyperplasia. II. Cognitive and behavioral studies. Behav Genet, 5(2), 175-188. Retrieved from http://www.ncbi.nlm.nih.gov/pubmed/1131146

McLennan, J. D., Lord, C., \& Schopler, E. (1993). Sex differences in higher functioning people with autism. J Autism Dev Disord, 23(2), 217-227. Retrieved from http://www.ncbi.nlm.nih.gov/pubmed/8331044

Meador, K. J., \& Loring, D. W. (2013). Risks of in utero exposure to valproate. JAMA, 309(16), 1730-1731. doi:10.1001/jama.2013.4001

Meaney, M. J., Stewart, J., \& Beatty, W. W. (1985). Sex-differences in social play - the socialization of sex-roles. Advances in the Study of Behavior, 15, 1-58. doi:Doi 10.1016/S0065-3454(08)60486-6

Meeker, N. D., Hutchinson, S. A., Ho, L., \& Trede, N. S. (2007). Method for isolation of PCR-ready genomic DNA from zebrafish tissues. Biotechniques, 43(5), 610, 612, 614. Retrieved from http://www.ncbi.nlm.nih.gov/pubmed/18072590

Mehta, M. V., Gandal, M. J., \& Siegel, S. J. (2011). mGluR5-antagonist mediated reversal of elevated stereotyped, repetitive behaviors in the VPA model of autism. PLoS One, 6(10), e26077. doi:10.1371/journal.pone.0026077

Mester, J. L., Tilot, A. K., Rybicki, L. A., Frazier, T. W., 2nd, \& Eng, C. (2011). Analysis of prevalence and degree of macrocephaly in patients with germline PTEN mutations and of brain weight in Pten knock-in murine model. Eur J Hum Genet, 19(7), 763-768. doi:10.1038/ejhg.2011.20

Meziane, H., Ouagazzal, A. M., Aubert, L., Wietrzych, M., \& Krezel, W. (2007). Estrous cycle effects on behavior of $\mathrm{C} 57 \mathrm{BL} / 6 \mathrm{~J}$ and $\mathrm{BALB} / \mathrm{cByJ}$ female mice: implications 
for phenotyping strategies. Genes Brain Behav, 6(2), 192-200.

doi:10.1111/j.1601-183X.2006.00249.x

Moldin, S. O., \& Rubenstein, J. L. R. (2006). Understanding Autism: From Basic

Neuroscience to Treatment. Boca Raton: Taylor \& Francis Group, LLC.

Mueller, B. R., \& Bale, T. L. (2007). Early prenatal stress impact on coping strategies

and learning performance is sex dependent. Physiol Behav, 91(1), 55-65.

doi:10.1016/j.physbeh.2007.01.017

Mueller, B. R., \& Bale, T. L. (2008). Sex-specific programming of offspring emotionality after stress early in pregnancy. J Neurosci, 28(36), 9055-9065.

doi:10.1523/JNEUROSCI.1424-08.2008

Muhle, R., Trentacoste, S. V., \& Rapin, I. (2004). The genetics of autism. Pediatrics, 113(5), e472-486. Retrieved from http://www.ncbi.nlm.nih.gov/pubmed/15121991

Narita, M., Oyabu, A., Imura, Y., Kamada, N., Yokoyama, T., Tano, K., . . . Narita, N. (2010). Nonexploratory movement and behavioral alterations in a thalidomide or valproic acid-induced autism model rat. Neurosci Res, 66(1), 2-6.

doi:10.1016/j.neures.2009.09.001

Neul, J. L., \& Zoghbi, H. Y. (2004). Rett syndrome: a prototypical neurodevelopmental disorder. Neuroscientist, 10(2), 118-128. doi:10.1177/1073858403260995

Nicholas, J. S., Charles, J. M., Carpenter, L. A., King, L. B., Jenner, W., \& Spratt, E. G. (2008). Prevalence and characteristics of children with autism-spectrum disorders. Ann Epidemiol, 18(2), 130-136. doi:10.1016/j.annepidem.2007.10.013 
Nordahl, C. W., Iosif, A. M., Young, G. S., Perry, L. M., Dougherty, R., Lee, A., . . Amaral, D. G. (2015). Sex differences in the corpus callosum in preschool-aged children with autism spectrum disorder. Mol Autism, 6, 26. doi:10.1186/s13229015-0005-4

Novak, C. M., Burghardt, Paul R., and Levine, James A. (2012). The use of a running wheel to measure activity in rodents: Relationship to energy balance, general activity, and reward. Neuroscience and Biobehavioral Reviews, 36, 1001-1014. doi:10.1016/j.neubiorev.2011.12.012

Oddi, D., Crusio, W. E., D'Amato, F. R., \& Pietropaolo, S. (2013). Monogenic mouse models of social dysfunction: implications for autism. Behav Brain Res, 251, 7584. doi:10.1016/j.bbr.2013.01.002

Page, D. T., Kuti, O. J., Prestia, C., \& Sur, M. (2009). Haploinsufficiency for Pten and Serotonin transporter cooperatively influences brain size and social behavior. Proc Natl Acad Sci U S A, 106(6), 1989-1994. doi:10.1073/pnas.0804428106

Palmen, S. J., van Engeland, H., Hof, P. R., \& Schmitz, C. (2004). Neuropathological findings in autism. Brain, 127(Pt 12), 2572-2583. doi:10.1093/brain/awh287

Palomba, S., Marotta, R., Di Cello, A., Russo, T., Falbo, A., Orio, F., . . La Sala, G. B. (2012). Pervasive developmental disorders in children of hyperandrogenic women with polycystic ovary syndrome: a longitudinal case-control study. Clin Endocrinol (Oxf), 77(6), 898-904. doi:10.1111/j.1365-2265.2012.04443.x

Park, J. J., Baum, M. J., Paredes, R. G., \& Tobet, S. A. (1996). Neurogenesis and cell migration into the sexually dimorphic preoptic area/anterior hypothalamus of the 
fetal ferret. J Neurobiol, 30(3), 315-328. doi:10.1002/(SICI)10974695(199607)30:3\&1t;315::AID-NEU1\&gt;3.0.CO;2-7

Patterson, P. H. (2006). Modeling features of autism in animals. In S. O. a. R. Moldin, John. L.R. (Ed.), Understanding autism: from basic neuroscience to treatment (pp. 277-302). Boca Raton, FL: Taylor \& Francis Group, LLC.

Patterson, P. H. (2009). Immune involvement in schizophrenia and autism: etiology, pathology and animal models. Behav Brain Res, 204(2), 313-321. doi:10.1016/j.bbr.2008.12.016

Pfaff, D. W., Rapin, I., \& Goldman, S. (2011). Male predominance in autism: neuroendocrine influences on arousal and social anxiety. Autism Res, 4(3), 163176. doi:10.1002/aur.191

Philip, R. C., Dauvermann, M. R., Whalley, H. C., Baynham, K., Lawrie, S. M., \& Stanfield, A. C. (2012). A systematic review and meta-analysis of the fMRI investigation of autism spectrum disorders. Neurosci Biobehav Rev, 36(2), 901942. doi:10.1016/j.neubiorev.2011.10.008

Phoenix, C. G., RW; Gerall, AA; and Young, WC. (1959). Organizing action of prenatally administered testosterone propionate on the tissues mediating mating behavior in the female guinea pig. Endocrinology, 65, 369-382.

Pilowsky, T., Yirmiya, N., Shulman, C., \& Dover, R. (1998). The Autism Diagnostic Interview-Revised and the Childhood Autism Rating Scale: differences between diagnostic systems and comparison between genders. J Autism Dev Disord, 28(2), 143-151. Retrieved from http://www.ncbi.nlm.nih.gov/pubmed/9586776 
Pinter, O., Beda, Z., Csaba, Z., and Gerendai, I. (2007). Differences in the onset of puberty in selected inbred mouse strains. Paper presented at the 9th European Congress of Endocrinology, Budapest, Hungary.

Prendergast, B. J., Onishi, K. G., \& Zucker, I. (2014). Female mice liberated for inclusion in neuroscience and biomedical research. Neurosci Biobehav Rev, 40, 1-5. doi:10.1016/j.neubiorev.2014.01.001

Putting gender on the agenda. (2010). Nature, 465(7299), 665. doi:10.1038/465665a

Qin, J., Suh, J. M., Kim, B. J., Yu, C. T., Tanaka, T., Kodama, T., . . Tsai, S. Y. (2007). The expression pattern of nuclear receptors during cerebellar development. Dev Dyn, 236(3), 810-820. doi:10.1002/dvdy.21060

Reichenberg, A., Gross, R., Weiser, M., Bresnahan, M., Silverman, J., Harlap, S., . . . Susser, E. (2006). Advancing paternal age and autism. Arch Gen Psychiatry, 63(9), 1026-1032. doi:10.1001/archpsyc.63.9.1026

Rice, W. R. (1988). Analyzing tables of statistical tests. Evolution, 43(1), 223-225.

Rossignol, D. A., \& Frye, R. E. (2012). A review of research trends in physiological abnormalities in autism spectrum disorders: immune dysregulation, inflammation, oxidative stress, mitochondrial dysfunction and environmental toxicant exposures. Mol Psychiatry, 17(4), 389-401. doi:10.1038/mp.2011.165

Roullet, F. I., \& Crawley, J. N. (2011). Mouse models of autism: testing hypotheses about molecular mechanisms. Curr Top Behav Neurosci, 7, 187-212. doi:10.1007/7854_2010_113 
Roullet, F. I., Lai, J. K., \& Foster, J. A. (2013). In utero exposure to valproic acid and autism--a current review of clinical and animal studies. Neurotoxicol Teratol, 36, 47-56. doi:10.1016/j.ntt.2013.01.004

Roullet, F. I., Wollaston, L., Decatanzaro, D., \& Foster, J. A. (2010). Behavioral and molecular changes in the mouse in response to prenatal exposure to the antiepileptic drug valproic acid. Neuroscience, 170(2), 514-522. doi:10.1016/j.neuroscience.2010.06.069

Russell, G., Steer, C., \& Golding, J. (2011). Social and demographic factors that influence the diagnosis of autistic spectrum disorders. Soc Psychiatry Psychiatr Epidemiol, 46(12), 1283-1293. doi:10.1007/s00127-010-0294-z

Ruta, L., Ingudomnukul, E., Taylor, K., Chakrabarti, B., \& Baron-Cohen, S. (2011). Increased serum androstenedione in adults with autism spectrum conditions. Psychoneuroendocrinology, 36(8), 1154-1163. doi:10.1016/j.psyneuen.2011.02.007

Rutter, M. (2005). Aetiology of autism: findings and questions. J Intellect Disabil Res, 49(Pt 4), 231-238. doi:10.1111/j.1365-2788.2005.00676.x

Rybakowski, F., Chojnicka, I., Dziechciarz, P., Horvath, A., Janas-Kozik, M., Jeziorek, A., ... Dunajska, A. (2016). The role of genetic factors and pre- and perinatal influences in the etiology of autism spectrum disorders - indications for genetic referral. Psychiatr Pol, 50(3), 543-554. doi:10.12740/PP/43234

Saito, Y. A. (2011). The role of genetics in IBS. Gastroenterol Clin North Am, 40(1), 4567. doi:10.1016/j.gtc.2010.12.011 
Schaafsma, S. M., \& Pfaff, D. W. (2014). Etiologies underlying sex differences in Autism Spectrum Disorders. Front Neuroendocrinol, 35(3), 255-271. doi:10.1016/j.yfrne.2014.03.006

Schmidtova, E. K., Silvia; Celec, Peter; Ficek, Andrej; and Ostatnikova, Daniela. (2010). Polymorphisms in Genes Involved in Testosterone Metabolism in Slovak Autistic Boys. Endocrinologist, 20(5), 245-249.

Schneider, T., Roman, A., Basta-Kaim, A., Kubera, M., Budziszewska, B., Schneider, K., \& Przewlocki, R. (2008). Gender-specific behavioral and immunological alterations in an animal model of autism induced by prenatal exposure to valproic acid. Psychoneuroendocrinology, 33(6), 728-740. doi:10.1016/j.psyneuen.2008.02.011

Schumann, C. M., Barnes, C. C., Lord, C., \& Courchesne, E. (2009). Amygdala enlargement in toddlers with autism related to severity of social and communication impairments. Biol Psychiatry, 66(10), 942-949. doi:10.1016/j.biopsych.2009.07.007

Schumann, C. M., Bloss, C. S., Barnes, C. C., Wideman, G. M., Carper, R. A., Akshoomoff, N., . . Courchesne, E. (2010). Longitudinal magnetic resonance imaging study of cortical development through early childhood in autism. $J$ Neurosci, 30(12), 4419-4427. doi:10.1523/jneurosci.5714-09.2010

Silverman, J. L., Yang, M., Lord, C., \& Crawley, J. N. (2010). Behavioural phenotyping assays for mouse models of autism. Nat Rev Neurosci, 11(7), 490-502. doi:10.1038/nrn2851 
Skuse, D. H. (2000). Imprinting, the X-chromosome, and the male brain: explaining sex differences in the liability to autism. Pediatr Res, 47(1), 9-16. Retrieved from http://www.ncbi.nlm.nih.gov/pubmed/10625077

Skuse, D. H., James, R. S., Bishop, D. V., Coppin, B., Dalton, P., Aamodt-Leeper, G., . . . Jacobs, P. A. (1997). Evidence from Turner's syndrome of an imprinted X-linked locus affecting cognitive function. Nature, 387(6634), 705-708. doi: $10.1038 / 42706$

Spring, S., Lerch, J. P., \& Henkelman, R. M. (2007). Sexual dimorphism revealed in the structure of the mouse brain using three-dimensional magnetic resonance imaging. Neuroimage, 35(4), 1424-1433. doi:10.1016/j.neuroimage.2007.02.023

Stolk, R. P., Rosmalen, J. G., Postma, D. S., de Boer, R. A., Navis, G., Slaets, J. P., . . . Wolffenbuttel, B. H. (2008). Universal risk factors for multifactorial diseases: LifeLines: a three-generation population-based study. Eur J Epidemiol, 23(1), 6774. doi:10.1007/s10654-007-9204-4

Swaab, D. F. (2004). Sexual differentiation of the human brain: relevance for gender identity, transsexualism and sexual orientation. Gynecol Endocrinol, 19(6), 301312. Retrieved from http://www.ncbi.nlm.nih.gov/pubmed/15724806

Swallow, J. G., Carter, P. A., \& Garland, T., Jr. (1998). Artificial selection for increased wheel-running behavior in house mice. Behav Genet, 28(3), 227-237. Retrieved from http://www.ncbi.nlm.nih.gov/pubmed/9670598

Tartaglia, N., Cordeiro, L., Howell, S., Wilson, R., \& Janusz, J. (2010). The spectrum of the behavioral phenotype in boys and adolescents 47,XXY (Klinefelter 
syndrome). Pediatr Endocrinol Rev, 8 Suppl 1, 151-159. Retrieved from http://www.ncbi.nlm.nih.gov/pubmed/21217607

The Jackson Laboratory. (2014). Body Weight Information - JAX® Mice Strain C57BL/6J (000664) Retrieved from http://jaxmice.jax.org/strain/000664.html

Tilot, A. K., Gaugler, M. K., Yu, Q., Romigh, T., Yu, W., Miller, R. H., .. . Eng, C. (2014). Germline disruption of Pten localization causes enhanced sex-dependent social motivation and increased glial production. Hum Mol Genet, 23(12), 32123227. doi:10.1093/hmg/ddu031

Tobet, S. A., Basham, M. E., \& Baum, M. J. (1993). Estrogen receptor immunoreactive neurons in the fetal ferret forebrain. Brain Res Dev Brain Res, 72(2), 167-180. Retrieved from http://www.ncbi.nlm.nih.gov/pubmed/8485841

Todd, R. D. a. H., J.J. (1993). Genetics of autism. CurrOpinPsychiatr 1993, 6, 486-488.

Tordjman, S., Somogyi, E., Coulon, N., Kermarrec, S., Cohen, D., Bronsard, G., . . . Xavier, J. (2014). Gene x Environment interactions in autism spectrum disorders: role of epigenetic mechanisms. Front Psychiatry, 5, 53. doi:10.3389/fpsyt.2014.00053

Tsutsui, K. (2008). Neurosteroids in the Purkinje cell: biosynthesis, mode of action and functional significance. Mol Neurobiol, 37(2-3), 116-125. doi:10.1007/s12035008-8024-1

Tuchman, R., Cuccaro, M., \& Alessandri, M. (2010). Autism and epilepsy: historical perspective. Brain Dev, 32(9), 709-718. doi:10.1016/j.braindev.2010.04.008 
van Rijn, S., \& Swaab, H. (2011). Vulnerability for psychopathology in Klinefelter syndrome: age-specific and cognitive-specific risk profiles. Acta Paediatr, 100(6), 908-916. doi:10.1111/j.1651-2227.2011.02289.x

Via, E., Radua, J., Cardoner, N., Happe, F., \& Mataix-Cols, D. (2011). Meta-analysis of gray matter abnormalities in autism spectrum disorder: should Asperger disorder be subsumed under a broader umbrella of autistic spectrum disorder? Arch Gen Psychiatry, 68(4), 409-418. doi:10.1001/archgenpsychiatry.2011.27

Volkmar, F. R., Szatmari, P., \& Sparrow, S. S. (1993). Sex differences in pervasive developmental disorders. J Autism Dev Disord, 23(4), 579-591. Retrieved from http://www.ncbi.nlm.nih.gov/pubmed/8106301

Wagner, G. C., Reuhl, K. R., Cheh, M., McRae, P., \& Halladay, A. K. (2006). A new neurobehavioral model of autism in mice: pre- and postnatal exposure to sodium valproate. J Autism Dev Disord, 36(6), 779-793. doi:10.1007/s10803-006-0117-y

Wang, S. S., Kloth, A. D., \& Badura, A. (2014). The cerebellum, sensitive periods, and autism. Neuron, 83(3), 518-532. doi:10.1016/j.neuron.2014.07.016

Wassink, T. H., Piven, J., \& Patil, S. R. (2001). Chromosomal abnormalities in a clinic sample of individuals with autistic disorder. Psychiatr Genet, 11(2), 57-63.

Retrieved from http://www.ncbi.nlm.nih.gov/pubmed/11525418

Weinstock, M. (2008). The long-term behavioural consequences of prenatal stress. Neurosci Biobehav Rev, 32(6), 1073-1086. doi:10.1016/j.neubiorev.2008.03.002 Werling, D. M., \& Geschwind, D. H. (2013). Understanding sex bias in autism spectrum disorder. Proc Natl Acad Sci U S A, 110(13), 4868-4869. doi:10.1073/pnas. 1301602110 
Werme, M., Messer, C., Olson, L., Gilden, L., Thoren, P., Nestler, E. J., \& Brene, S. (2002). Delta FosB regulates wheel running. J Neurosci, 22(18), 8133-8138. Retrieved from http://www.ncbi.nlm.nih.gov/pubmed/12223567

White, S. W., Oswald, D., Ollendick, T., \& Scahill, L. (2009). Anxiety in children and adolescents with autism spectrum disorders. Clin Psychol Rev, 29(3), 216-229. doi:10.1016/j.cpr.2009.01.003

Whitehouse, A. J., Mattes, E., Maybery, M. T., Dissanayake, C., Sawyer, M., Jones, R. M., .. . Hickey, M. (2012). Perinatal testosterone exposure and autistic-like traits in the general population: a longitudinal pregnancy-cohort study. J Neurodev Disord, 4(1), 25. doi:10.1186/1866-1955-4-25

Yeargin-Allsopp, M., Rice, C., Karapurkar, T., Doernberg, N., Boyle, C., \& Murphy, C. (2003). Prevalence of autism in a US metropolitan area. JAMA, 289(1), 49-55. Retrieved from http://www.ncbi.nlm.nih.gov/pubmed/12503976 


\section{APPENDIX}

Approval to copy and redistribute the contents of Chapter 2 (published in the Journal of Ethology) as per the publishers policy: "Author retains the right to use his/her article for his/her further scientific career by including the final published journal article in other publications such as dissertations and postdoctoral qualifications provided acknowledgement is given to the original source of publication." No changes were made to the paper in this dissertation. 


\section{CURRICULUM VITAE \\ Elizabeth A. Gordon \\ Department of Biology, Life Sciences Rm 139 \\ University of Louisville \\ Louisville KY 40292 \\ Phone: (270) 705-4948 \\ elizabethgordon_23@yahoo.com}

\section{EDUCATION}

UNIVERSITY OF LOUISVILLE

Louisville, $\mathrm{KY}$

Degree: Doctor of Philosophy

Concentration: Biology

Qualifying Exam Passed: July 2013

Expected Graduation Date: December 2016

Overall G.P.A. 4.00/4.00

UNIVERSITY OF LOUISVILLE

August 2008-May 2010

Louisville, KY

Degree: Master of Public Health (CEPH accredited)

Concentration: Health Promotion and Behavioral Sciences

Graduation Date: May 2010

August 2012-Present

MURRAY STATE UNIVERSITY

Overall G.P.A. 3.98/4.00

Murray, KY

Degree: Bachelor of Science

Major: Biology

Graduation Date: May 2008

August 2004-May 2008

Honor: Summa Cum Laude

Minor: Chemistry

Overall G.P.A. 3.86/4.00

\section{CERTIFICATION}

NATIONAL BOARD OF PUBLIC HEALTH EXAMINERS

Certification in Public Health

ID \#: 5887

August 2010

PROFESSIONAL WORK EXPERIENCE

VANDERBILT UNIVERSITY MEDICAL CENTER August 2016-Present

Nashville, TN

Health Policy/Services Analyst II

Vanderbilt Memory \& Alzheimer's Center

- Formulate grant applications and manuscript preparation for a large NIH clinical research portfolio

- Assist Center Director with all research dissemination

- Collaborate with biostatisticians to facilitate management and interpretation numerous data sets

- Serve as a resource to an interdisciplinary team of more than 20 to faculty, staff, and trainees 
Louisville, $\mathrm{KY}$

$\mathrm{PhD}$ Candidate

- Compose grant applications/research proposals to local and national organizations to fund research projects

- Manage short and long-term research priorities to meet deadlines

- Analyze large data sets to interpret results of studies

- Present research findings at local and national meetings to both scientific and lay audiences

- Draft scientific/technical articles for publication in peer-reviewed journals

\section{UNIVERSITY OF LOUISVILLE}

July 2013-June 2015

Louisville, KY

Graduate Teaching Assistant

- Teach multiple sections of Biology 104 laboratory to encourage an understanding of biological science; responsible for over 100 students

- Prepare and deliver lectures over basic concepts, such as the scientific method, genetics, physiology, nutrient cycling, and biological molecules

- Create, administer, and grade quizzes, lab reports, and exams

- Maintain a classroom dynamic that supports learning

NORTON CANCER INSTITUTE

August 2010-August 2012

Louisville, $\mathrm{KY}$

NCCCP Program Specialist

National Cancer Institute Community Cancer Centers Program

- Assist with formulating, implementing, and administering program goals and priorities

- Prepare quarterly reports and other statements, justification, and/or supporting documentation to the National Cancer Institute

- Lead proposal preparation for NCCCP 2 year extension RFP and National Cancer Institute Community Oncology Research Program (NCORP) RFP

- Arrange, promote and organize conferences, staff trainings, meetings

- Facilitate communication between all NCCCP departments

- Assemble/coordinate NCCCP programs in the areas of quality of care, clinical trials, survivorship \& palliative care, biospecimens, and communication

\section{CARDIOVASCULAR INNOVATIONS INSTITUTE}

Louisville, KY

May 2009-July 2010

Lab Assistant

- Assist supervisors in experiments and tissue culture work

- Make solutions and other items needed for experiments

- Follow protocols to complete assigned tasks

- Search companies and catalogs to order various supplies

- Organize and maintain ordering spreadsheets, company information folders, and other documents

- Perform numerous tasks in a timely and professional manner

YMCA OF GREATER LOUISVILLE Louisville, KY

June 2009-August 2010

Diabetes Prevention Program Facilitator

- Motivate pre-diabetics to change unhealthy behavioral habits

- Prepare materials and lessons for each session

- Facilitate group discussions towards positive lifestyle choices

- Analyze participants' progress and tailor method to meet their specific needs 
UNIVERSITY OF LOUISVILLE HOSPITAL

June 2009-April 2010

Employee Health \& Wellness Intern

- Assist with planning and implementation of wellness events

- Evaluate Smoking Cessation Program

- Compose health promotion articles for Employee Newsletter

- Compile and analyze data from new employee health survey

\section{RESEARCH INTERESTS}

Perinatal organization of the brain by gonadal hormones

Sex bias in autism spectrum disorder

\section{RESEARCH EXPERIENCE}

Sex differences in murine wheel running behavior July 2013-December 2014

Valproic acid dosing regimens and behavioral assessment March 2015-May 2015

Sex differences, perinatal testosterone in autism mouse model Sept. 2015-November 2016

\section{ORGANIZATIONALS \& AFFILIATIONS}

SIGS GRADUATE STUDENT AMBASSADOR

SOCIETY FOR NEUROSCIENCE

KENTUCKY ACADEMY OF SCIENCE

NASC HONORS SOCIETY

KENTUCKY PUBLIC HEALTH ASSOCIATION

SPARK P.E. RESEARCH DATA COLLECTOR

ALPHA LAMBDA DELTA HONORS SOCIETY

Secretary from January 2005-January 2006

TRI BETA BIOLOGICAL HONORS SOCIETY
August 2014-Present

August 2014-Present

November 2013-Present

January 2009-Present

September 2008-Present

September 2008-May 2009

January 2005-Present

August 2005-Present

\section{CONTINUING EDUCATION}

GRADUATE TEACHING ASSISTANT ACADEMY $\quad$ September 2013-April 2014

\section{GRANTS \& AWARDS}

SFN LOUISVILLE CHAPTER OUTSTANDING NEUROSCIENCE TRAINEE AWARD

April 2016

$\$ 500$

GRADUATE STUDENT COUNCIL RESEARCH AWARD

BIOLOGY DEPARTMENT FUNDING ALLOCATION

RESEARCH INITIATION GRANT

C. Corbitt and M. Casanova, PIs

RESEARCH AND CREATIVE ACTIVITIES GRANT

GRADUATE STUDENT COUNCIL TRAVEL AWARD

UNIVERSITY OF LOUISVILLE FELLOWSHIP

January 2016-June $2016 \quad \$ 300$

August 2015

$\$ 1000$

January 2014-June $2015 \quad \$ 3,085$

May 2013-April $2014 \quad \$ 500$

November 2013

$\$ 350$

July 2015-June 2016

$\$ 36,000$

July 2012-June $2013 \quad \$ 36,000$

TROVER RURAL SCHOLAR PROGRAM

July 2007

$\$ 4,000$

July 2006

\section{PUBLICATIONS \& PRESENTATIONS}

Gordon, E., Bryan, E., Collins, M.D., Dugan, S., Duvall, D., Hamilton, K., Jacob, A., Karim, T., Obrik-Uloho, O., Pena-Calderin, E., and Corbitt, C. Evaluating sex differences 
and the effect of perinatal testosterone in the VPA mouse model of autism. Society for Neuroscience Annual Conference, San Diego, California, November 15, 2016.

Gordon, E., Bamji, S., Bencker, K., Patel, D., and Corbitt, C. Investigation of sex differences in wheel running and social behavior in pre-pubertal mice. Neuroscience Day, University of Louisville, Louisville, Kentucky, April 23, 2015.

Gordon, E., Bamji, S., Bencker, K., Patel, D., and Corbitt, C. Investigation of sex differences in wheel running and social behavior in pre-pubertal mice. Society for Neuroscience Annual Conference, Washington, D.C., November 18, 2014.

Gordon, E. and Corbitt, C. Investigation of pre-pubertal sex differences in wheel running and social behavior in mice. Neuroscience Day, University of Louisville, Louisville, Kentucky, April 21, 2014.

Gordon, E. and Corbitt, C. Investigation of pre-pubertal sex differences in wheel running and social behavior in mice. Kentucky Academy of Science $99^{\text {th }}$ Annual Meeting, Morehead State University, Morehead, Kentucky, November 9, 2013.

Stallings, H., Gansauer, L., Castro, K., Clauser, S., Kadlubek, P., Siegel, R. and Gordon, E. Addressing Nursing Sensitive Patient Care: The National Cancer Institute (NCI) Community Cancer Centers Program (NCCCP) Quality Improvement Initiative. Oncology Nursing Society $37^{\text {th }}$ Annual Conference, New Orleans, Louisiana, May 5, 2012.

Terzic, J, Gordon, E., Hembree, T, Brooks, S. Enhancing Community Partnership to reduce cancer disparities: A supported mini grant approach. Kentucky Public Health Association Annual Conference. Louisville, Kentucky, March 29, 2012.

Roberts, C., Gordon, E., Williams, S., Davis, M.H. Quality Study: Timing of referral to Hospice. Psycho-Oncology. Volume, 21, Issue Supplement s1. February 2012.

Roberts, C., Gordon, E., Williams, S., Davis, M.H. Quality Study: Timing of referral to Hospice. American Psychosocial Oncology Society $9^{\text {th }}$ Annual Conference. Miami, Florida, February, 24, 2012.

Gordon, E. Participation in the National Cancer Institute Community Cancer Centers Program (NCCCP). Kentucky Public Health Association Annual Conference 